\title{
A SIMULATION ALGORITHM CAPABLE OF MODELLING SPATIAL IMPACT POINTS FROM THE NEUTRALIZATION OF AN IMPROVISED EXPLOSIVE DEVICE
}

\author{
By \\ Christopher Chan \\ Bachelor of Science \\ Computer Science, \\ Ryerson University 2013 \\ Master of Science \\ Computer Science, \\ Ryerson University 2015
}

\author{
A dissertation \\ presented to Ryerson University \\ in partial fulfillment of the \\ requirements for the degree of \\ Doctor of Philosophy \\ in the program of \\ Computer Science
}

Toronto, Ontario, Canada, 2018

(C) Christopher Chan, 2018 


\section{Author's Declaration}

I hereby declare that I am the sole author of this dissertation. This is a true copy of the dissertation, including any required final revisions, as accepted by my examiners.

I authorize Ryerson University to lend this dissertation to other institutions or individuals for the purpose of scholarly research.

I further authorize Ryerson University to reproduce this dissertation by photocopying or by other means, in total or in part, at the request of other institutions or individuals for the purpose of scholarly research.

I understand that my dissertation may be made electronically available to the public. 


\title{
Abstract
}

\section{A SIMULATION ALGORITHM CAPABLE OF MODELLING SPATIAL IMPACT POINTS FROM THE NEUTRALIZATION OF AN IMPROVISED EXPLOSIVE DEVICE}

\author{
Christopher Chan
}

\section{Doctor of Philosophy, Computer Science, Ryerson University, 2018}

An improvised explosive device (IED) is a bomb constructed from unknown materials, often concealed, such as inside an innocuous container, and deployed in unconventional ways resulting in a potentially deadly weapon. Public safety personnel such as Explosive Disposal Units (EDUs), are trained in the safe handling of explosives and the threats posed by IEDs. One method of neutralizing a suspect IED is to use water fired from a high-powered dispersion weapon commonly known as a disrupter cannon. Our research proposes an algorithm for developing an IED neutralization simulation that can emulate real-world physical effects of the successful neutralization of an IED without danger to the public or first responders.

This algorithm includes 6 methodologies with the goal of providing EDU with additional information on the potential physical dispersion of the components of an IED and any major points of impact (splatter) and possible actionable intelligence on the pose and direction of a disrupter cannon for a successful neutralization of an IED. We have developed a prototype simulation based on this algorithm and evaluated the simulation with an appropriate real-world disrupter and compared the real-world splatter to our simulation's splatter.

We argue systems developed with our algorithm may provide relevant information directly from the simulation and can be accurately used to analyze particle dispersion for the purposes of augmenting EDU IED neutralization processes. 


\section{Acknowledgements}

I would like to thank my gracious wife, Jessica Lin Zhang, for her relentless love and support. She went above and beyond to be ever so patient and tolerating me at my very best and at my very worst. I dedicate my hard work and zest for life to her and our baby girl, Isabelle.

I would like to thank my supervisor Dr. Alexander Ferworn for his direction and support. Dr. Ferworn introduced me to a great niche in computer science for the field of robotics for computational public safety and provided tremendous guidance, support and aid throughout my studies.

I would like to acknowledge the support I have received from the following groups and individuals. Without their help, my work would have not been possible:

Craig Dunfield and Mark Running of the Durham Regional Police Service for their advice on neutralization processes and for their help on gathering essential data for my final experiments.

Andy Olesen of the Halton Regional Police Service for his expert advice on neutralization equipment and EDU tactics.

The Emergency Task Force (ETF) of the Toronto Police Service (TPS) for the use of their facilities and equipment--especially the assistance of Explosive Force Entry Coordinator Peter Svitak, whose explanations and guidance helped me understand a very unfamiliar world.

Sunnybrook Hospital for the use of their medical imaging systems, especially Dr. Lee Chin who did not mind when I asked him to help me scan and process inert explosives in his hospital. 
The Network-Centric Applied Research Team (N-CART) for the advice, pressure and assistance provided to me by my peers and advisors, Rodney Yip, Jimmy Tran, Alex Ufkes, Dalia Hanna, Christopher Kong, Scott Herman, Nhan Tran, and Ben Waismark. Also, Tyler Zannon, for his assistance in scanning and importing 3D models into the Unity game engine and assistance in simulation programming.

Finally, I would like to thank my family, Dora, Elvic, Michael, Angela, Devin, Heidi, Vincent, Lana, Jacqueline, and Josephine for being there every step of the way and a constant source of support, without their gentle care and love, I would not be the person that I am today. 


\section{TABLE OF CONTENTS}

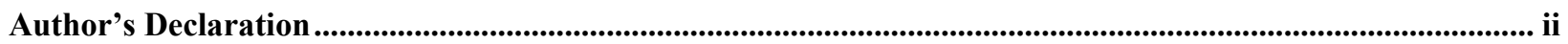

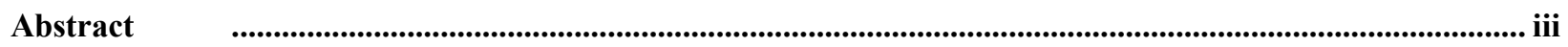

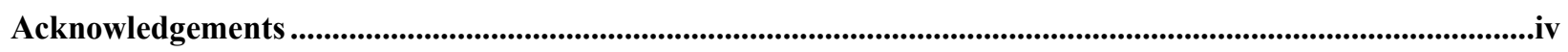

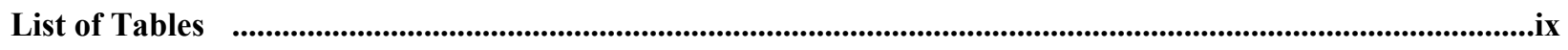

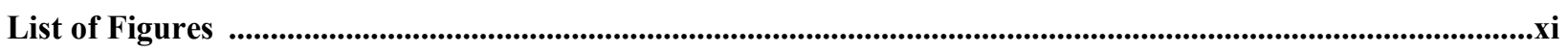

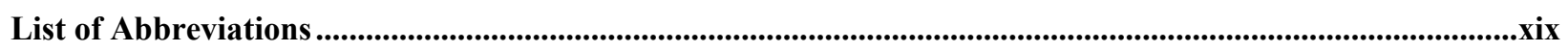

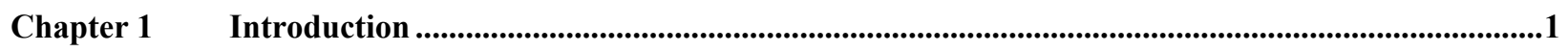

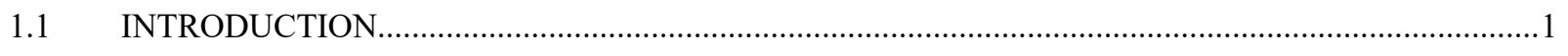

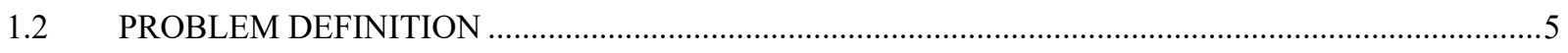

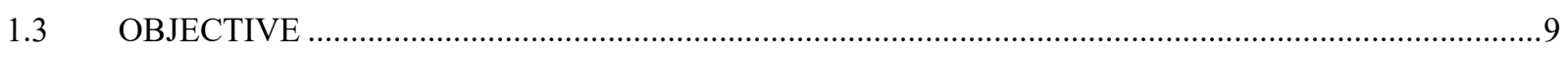

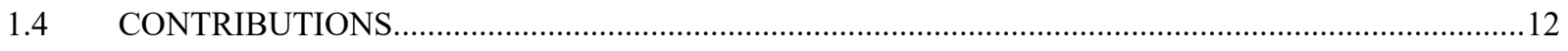

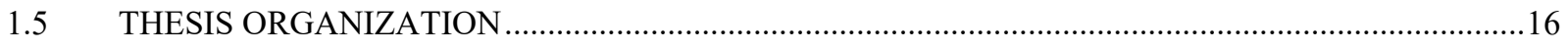

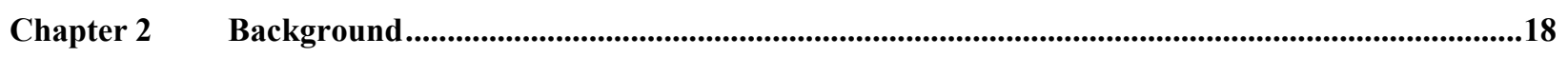

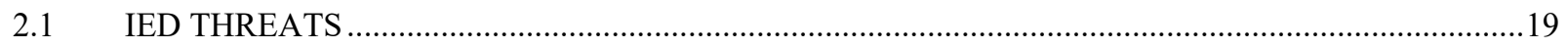

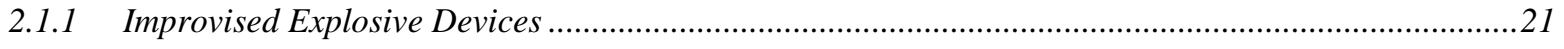

2.1.2 Mine Warfare ...........................................................................................................................25

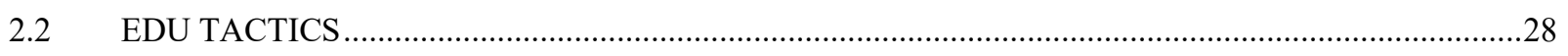

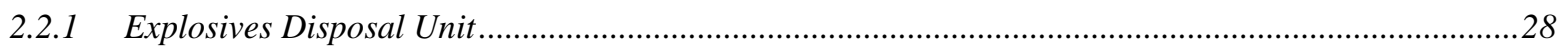

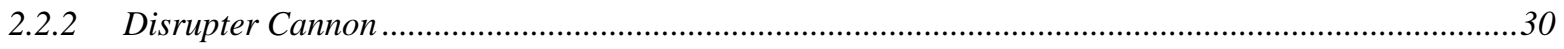

2.2.3 EDU Scanning Equipment .............................................................................................................33

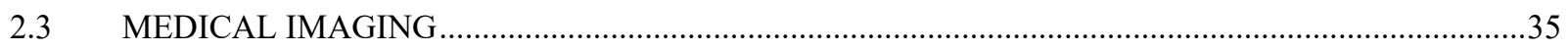

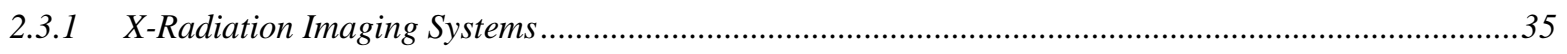

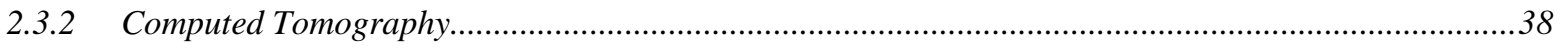

2.3.3 Imaging Techniques for Threat Objects.....................................................................................

2.3.4 Other Imaging Systems ..............................................................................................................43

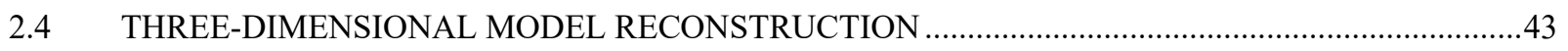

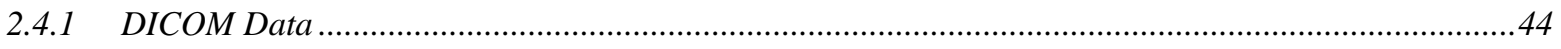

2.4.2 3D Model Reconstruction using DICOM Data...................................................................................44 
2.4.3 3D Model Reconstruction using Multiple Images..............................................................................45

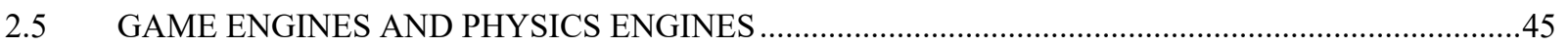

2.5.1 Unity Game Engine - Game/Simulation Development Platform .........................................................47

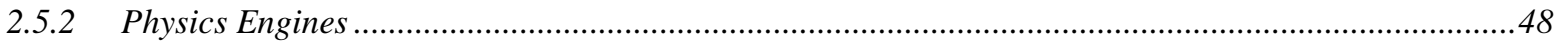

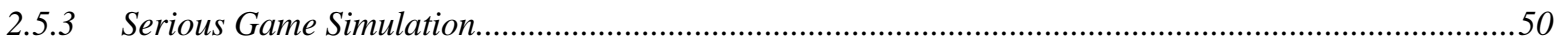

2.6 HIGH VELOCITY PROJECTILE IMPACT SIMULATION …...........................................................

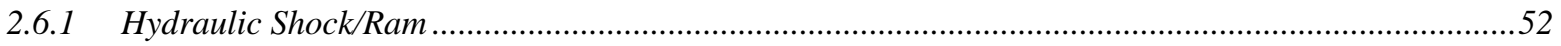

2.6.2 Particle Momentum Explosive Effects ............................................................................................52

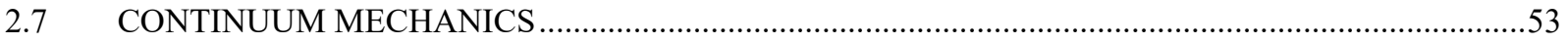

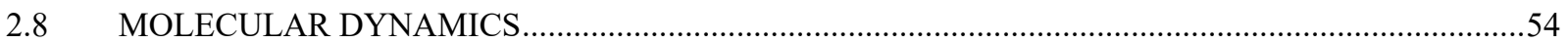

Chapter $3 \quad$ Technical Approach …........................................................................................................................57

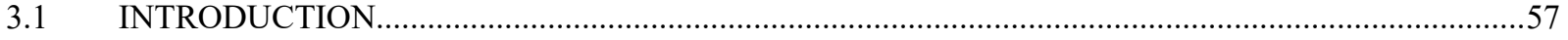

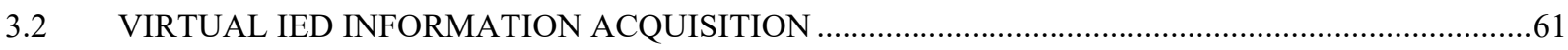

3.2.1 Virtual Improvised Explosive Device Creation...............................................................................61

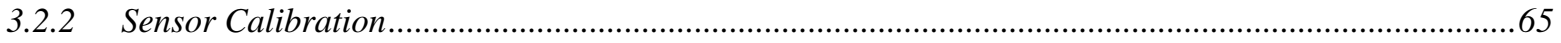

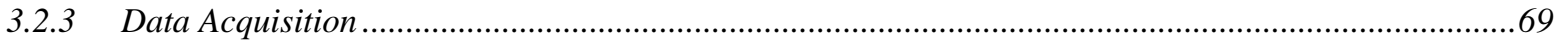

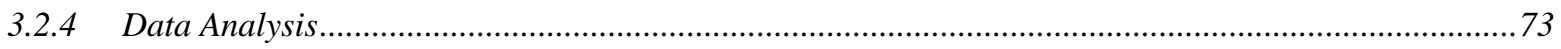

3.3 SERIOUS GAME ENGINE MODEL AND MESH CONFIGURATION …...........................................79

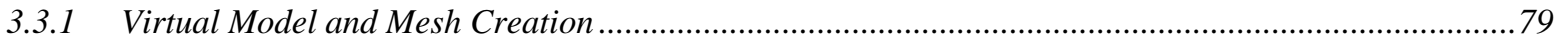

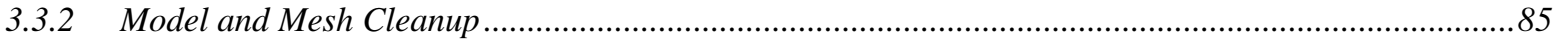

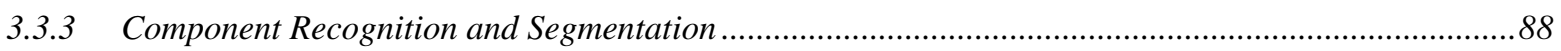

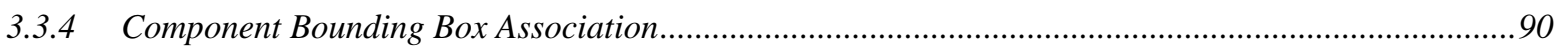

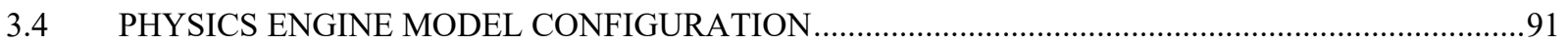

3.4.1 Molecular Dynamics Implementation ..............................................................................................91

3.4.2 Real-world Physical Attribute Implementation...............................................................................94

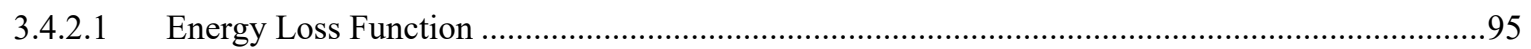

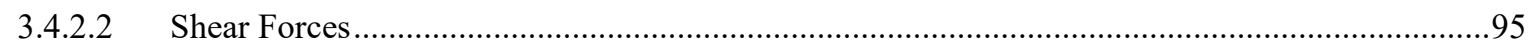

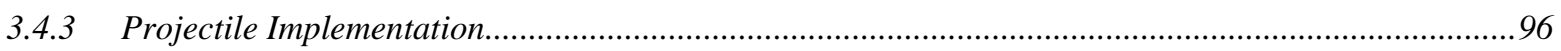

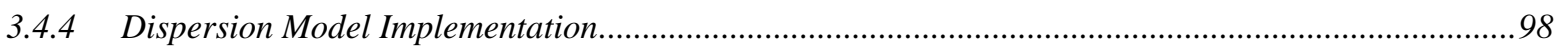

3.4.5 Game Actor Implementation ......................................................................................................100

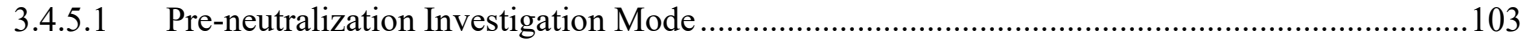

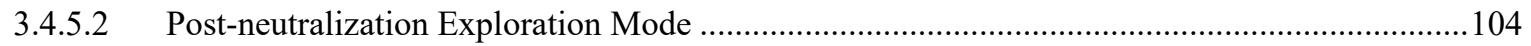

Chapter $4 \quad$ Experimental Results ......................................................................................................................106

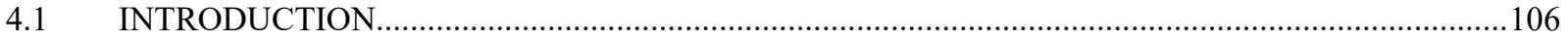




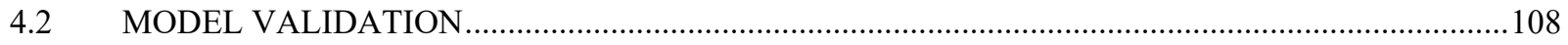

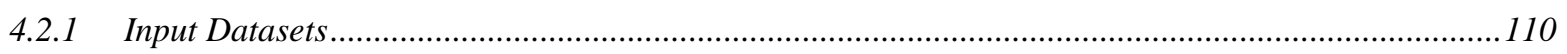

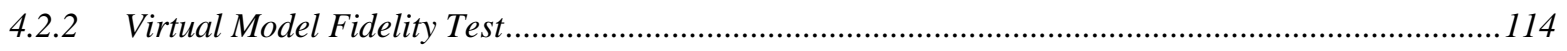

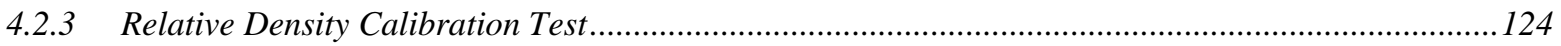

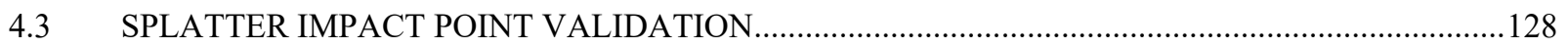

4.3.1 Single Projectile to One Mass.......................................................................................................128

4.3.2 Multiple Projectiles to Multiple Masses …...............................................................................136

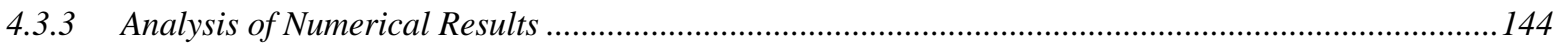

Chapter 5 Conclusion and Future Work ..................................................................................................................146

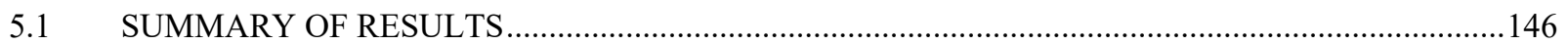

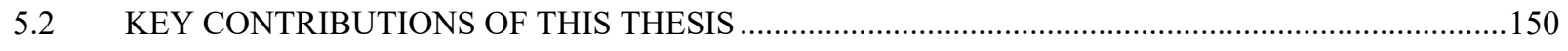

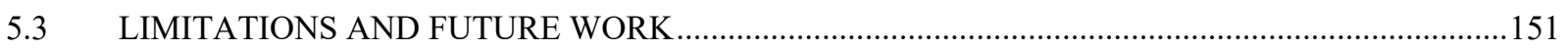

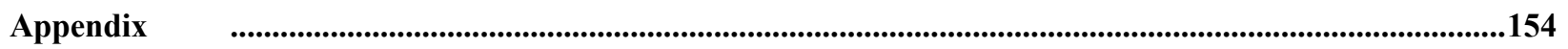

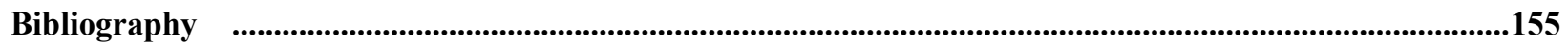




\section{List of Tables}

\#

Description

Page

Table 2.1 A noncomprehensive list of IED attacks in different locations and 20 different times.

Table 2.2 A noncomprehensive ID classification matrix of legacy IEDs and their typical means of initiation [18].

Table 2.3 Overview of types of explosives and their common uses, common form and the situation where such explosives were used [2].

Table 3.1 The common substances and their densities used in calibrating the CT 66 sensor.

Table 3.2 Head and neck parameters taken from the standard AAPM head and neck protocol, optimized for dense objects.

Table 3.3 The value obtained from the average of HSV pixel segment of the substance.

Table 3.4 The values obtained from the average of HSV pixel segment of each inert explosive.

Table 4.1 Comparison of exterior dimensions of IED containers.

Table 4.2 Comparison of exterior dimensions of $350 \mathrm{ml}$ water bottle containers with different substances.

Table 4.3 Comparison of exterior dimensions of inert explosives.

Table 4.4 Comparison of dimensions of IED components. 
Table 4.5 Photometric brightness values obtained using CT scanner 1 for common substances shown in 3 different scans with slightly modified parameters.

Table 4.6 Photometric brightness values obtained using CT scanner 2 for common substances shown in 3 different scans with slightly modified parameters.

Table 4.7 Photometric brightness values obtained using CT scanner 3 for common substances shown in 3 different scans with slightly modified parameters.

Table 4.8 This table shows the validation results of scanning an inert nitro dynamite on 3 different CT scanners, the PBV obtained from each scanner is shown in the rightmost column, and the density that each PBV is associated with is shown in the middle column.

Table 4.9 Our test equipment shown here includes a paintball gun, a pellet chronographer and HD video cameras.

Table 4.10 A view of the results comparing the percentages from the actual 138 experiment and the simulated model.

Table 4.11 Validation results of the real-world test to the simulation's output. The 145 rightmost column shows the difference between the two results. 


\section{List of Figures}

\#

Description

Page

Figure 1.1 An overview of the approximate location of the IED in the foyer of one 1 of the venue's exits [1].

Figure 1.2 Various IED contraptions, constructed in ways other than in conventional military action $[2-6]$

Figure 1.3 An image depicting two EDU personnel positioning and arming a disrupter cannon.

Figure 1.4 Typical construction of an IED.

Figure 2.1 An example of an armed Improvised Explosive Device [6].

Figure 2.2 Typical triggering mechanisms for explosive-type IEDs found in mine warfare [7].

Figure 2.3 Typical construction of a switch IED using a pressure mechanism [7]. 26

Figure 2.4 A standard method of connecting a mechanism to a charge [7].

Figure 2.5 A 20mm Recoilless MKIII Waterjet disrupter cannon by a defense supplier company [8].

Figure 2.6 A bomb containment vessel designed to direct an explosive blast upward. $\quad 30$

Figure 2.7 The schematic for a device for disrupting IEDs, filed under patent “US7481146B2” [9].

Figure 2.8 A shell casing of the propellant (left) and a number depicting the charge of the propellant (right). 
Figure 2.9 A 2D x-ray scan of a mock IED from EDU scanning equipment (left), a

flat circular lead coin is placed beside the scan for reference, the darker the intensity of the image, the denser the material (right).

Figure 2.10 A diagram depicting different applications that use different parts of the $\mathrm{X}$-ray spectrum [10].

Figure 2.11 Image slices of a brain from a MRI scan, similar to a CT scan [11]. 40

Figure 2.12 Examples of serious games developed for healthcare: serious game for surgery (top-left), serious game for pharmacy (top right), and serious game for nursing (bottom) [12].

Figure 2.13 Pressure map simulation of a projectile at a velocity of $900 \mathrm{~m} / \mathrm{s}$ [13] in a simulated closed fuel tank.

Figure 2.14 A computationally efficient rendering of a model represented by 4147 non-macroscopic particles (top right) [14].

Figure 3.1 A flowchart depicting the creation of virtual IEDs from real-world suspect 60 IEDs to the implementation of accurate physics-based models leading to a serious game simulation.

Figure 3.2 Revealed mock IED components in cardboard containers. 1. Metal/plastic suitcase with a cell phone (triggering mechanism) and 2-6. Cardboard container with IED components with varying types of material (for example metal, plastic, glass, or wood) in a variety of arrangements so the IEDs are random and improvised. 
Figure 3.3 From left to right, the liquids shown are honey, corn syrup, detergent, water, vegetable oil, lamp oil and rubbing alcohol, each chosen for their distinct densities.

Figure $3.4 \quad \# 1$. Semtex 10 Assembly. \#2. Nitro dynamite. \#3. M112 block (C-4) 65 Assembly. \#4. PETN. \#5. TNT cast booster. \#6. Smokeless black powder.

Figure 3.5 A sample treatment planning screenshot of Pinnacle by Philips [15], often cluttered with tedious and repetitive steps and parameters, requiring significant planner/physician interaction. Plan quality varies depending on the experience of the operator.

Figure 3.6 An image of the Philips Big Bore CT sensor with the target package of substances being scanned.

Figure 3.7 A diagram of a CT scanning machine [16].

Figure 3.8 3D representation of the suitcase and support platform from DICOM data.

Figure 3.9 3D representation of box 3 and 6 from DICOM data.

Figure 3.10 3D representation of box 2, 4 and 5 from DICOM data.

Figure 3.11 A 2D cross sectional output of a CT scan of the following household common substances: left to right: the liquids shown are honey, corn syrup, detergent, water, vegetable oil, lamp oil and rubbing alcohol, each showing up on the scan as different light intensities as a result of their different densities.

Figure 3.12 Three orthogonal views of an image slice from 6 inert explosives.

Figure 3.13 A representation of a mock IED in a cross sectional views shown in 3D Slicer. 
Figure 3.14 Dark streaks or thin bright and dark streaks due to metal artifact that commonly (21\% chance of occurrence in scans) appear in CT scanning due to the metal itself, or beam hardening, scatter effects and Poisson noise [17].

Figure 3.15 Artifacts (streaks) that appear on 3D slicer after creating 3D virtual models from DICOM data.

Figure 3.16 3D virtual models of EOD objects - top left: EDU bomb suit - top right: disrupter cannon - bottom left: EOD robot with camera and arm - bottom right: a suspicious package housing an IED.

Figure 3.17 A screenshot of Blender showing the interior of a container housing an IED. The front two cylindrical objects are virtual representations of wooden sticks simulating explosives.

Figure 3.18 These images describe non-manifold geometries which, in mesh 86 topology, manifold objects are usually 2-manifold, and these geometries have edges that are connected by 0,1 or 3 faces.

Figure 3.19 The resulting mesh after the hole filling algorithm is applied. All nonmanifold geometry is excluded.

Figure 3.20 A screenshot of Aperio. The container is modified using a mechanical tool to reveal the interior and to separate critical IED components of interest.

Figure 3.21 The mesh shown in Aperio after using a cutter to view objects that was occluded by the container.

Figure 3.22 A bounding box assigned to a critical component in the simulation. 
Figure 3.23 A representation of object disintegration into finite elements [18]. 91

Figure 3.24 A magnified view from a Unity scene of multiple game objects after the 92 mesh chunk algorithm is applied.

Figure 3.25 A reintegrated form of a 3D scanned model bag housing inert explosives. 93

Figure 3.26 This diagram represents the Newtonian collision interaction between two 94 particles and the elastic deformation represented by the small overlap between particles.

Figure 3.27 A scene in the simulation after firing the disrupter at components. 98

Figure 3.28 Multiple spherical unity game objects that are grouped together 99 representing shrapnel.

Figure 3.29 Left: Simulation of a particle dispersion in action. Right: Real-world 102 experiment of projectile impact resulting in dispersion.

Figure 3.30 Simulation console indicating the percentage of a sensor grid affected by particles.

Figure 3.31 Exploration of the possible dispersion area and points of impact of 105 particles after a successful neutralization of an IED occurs.

Figure 4.1 The algorithm's last phase: validate the model and functionality and also to test the serious game.

Figure 4.2 This dataset is shown in 3 coronal views (top) and 3D reconstructed view (bottom) of substances with distinct densities.

Figure 4.3 The fourth dataset shown in 3 coronal views (top) and 3D reconstructed view (bottom) of inert explosives consisting of semtex 10 assembly, nitro 
dynamite, M112 block (C-4) assembly, PETN, TNT cast booster, and smokeless black powder - housed in cardboard boxes.

Figure 4.4 Distance tool from the Unity asset store to measure game objects in the scene.

Figure 4.5 Differences in dimensions of the exterior of IED containers.

Figure 4.6 Differences in dimensions of exterior dimensions of $350 \mathrm{ml}$ water bottle containers with different substances.

Figure 4.7 Differences in dimensions of exterior dimensions of inert explosives.

Figure 4.8 Differences in dimensions of IED components.

Figure 4.9 Sensor grid pad labeled starting from top left as grid A1 to E5 bottom right.

Figure 4.10 Left: The target object as a customized Unity particle model. Right: Realworld target object.

Figure 4.11 Simulation console indicating the percentage of a sensor grid affected by particles.

Figure 4.12 Left: Simulation of a particle dispersion in action. Right: Real-world experiment of projectile impact resulting in dispersion.

Figure 4.13 The 'bowl effect' visual cue from a projectile impact.

Figure 4.14 A $29 \mathrm{~mm}$ Neutrex waterjet disrupter cannon used for our real-world experiments.

Figure 4.15 An 11 by 18 sensor grid pad used to capture the percentage of splatter on each grid after a dispersion of components from a target.

Figure 4.16 Real-world experiment of a disrupter cannon targeting a water balloon. 
Figure 4.17 Real-world experiment of a disrupter cannon targeting two water 140 balloons.

Figure 4.18 Real-world experiment of a disrupter cannon targeting an occluded half- 140 full windshield water container.

Figure 4.19 Real-world experiment of a disrupter cannon targeting multiple objects, one of which is an inert nitro dynamite. 


\section{Appendix}

A. Mesh Chunk Splitting Algorithm.....................................................164 


\section{List of Abbreviations}

3D

CT

CM

EDU

EOD

IED

HRAM

MD

MRI

RF

USAR
3-Dimensional

Computed Tomography

Continuum Mechanics

Explosive Disposal Unit

Explosive Ordinance Disposal

Improvised Explosive Device

Hydrodynamic Ram

Molecular Dynamics

Magnetic Resonance Imaging

Radio Frequency

Urban Search and Rescue 


\section{Chapter 1 Introduction}

\subsection{Introduction}

On May 22nd, 2017, a famous pop star had just performed her final song for the night, and children as young as eight were starting to exit the famous Manchester Arena. The Manchester Arena is a public space having the largest seating capacity of any indoor venue in the United Kingdom. That night, approximately 14,200 people attended the concert.

At one of the busiest exits from the arena, was the foyer - a housing for the box office and a path that lead to a car park and Victoria Train Station. In the middle of this foyer, stood a 22 year old man with an innocuous backpack. Inside was concealed an armed Improvised Explosive Device made more lethal by containing nuts and bolts intended as shrapnel. The man waited for the concert goers amid waiting parents.

When the bomb detonated the resulting blast was so fierce that it blew people off their feet and shook the floor of the arena.

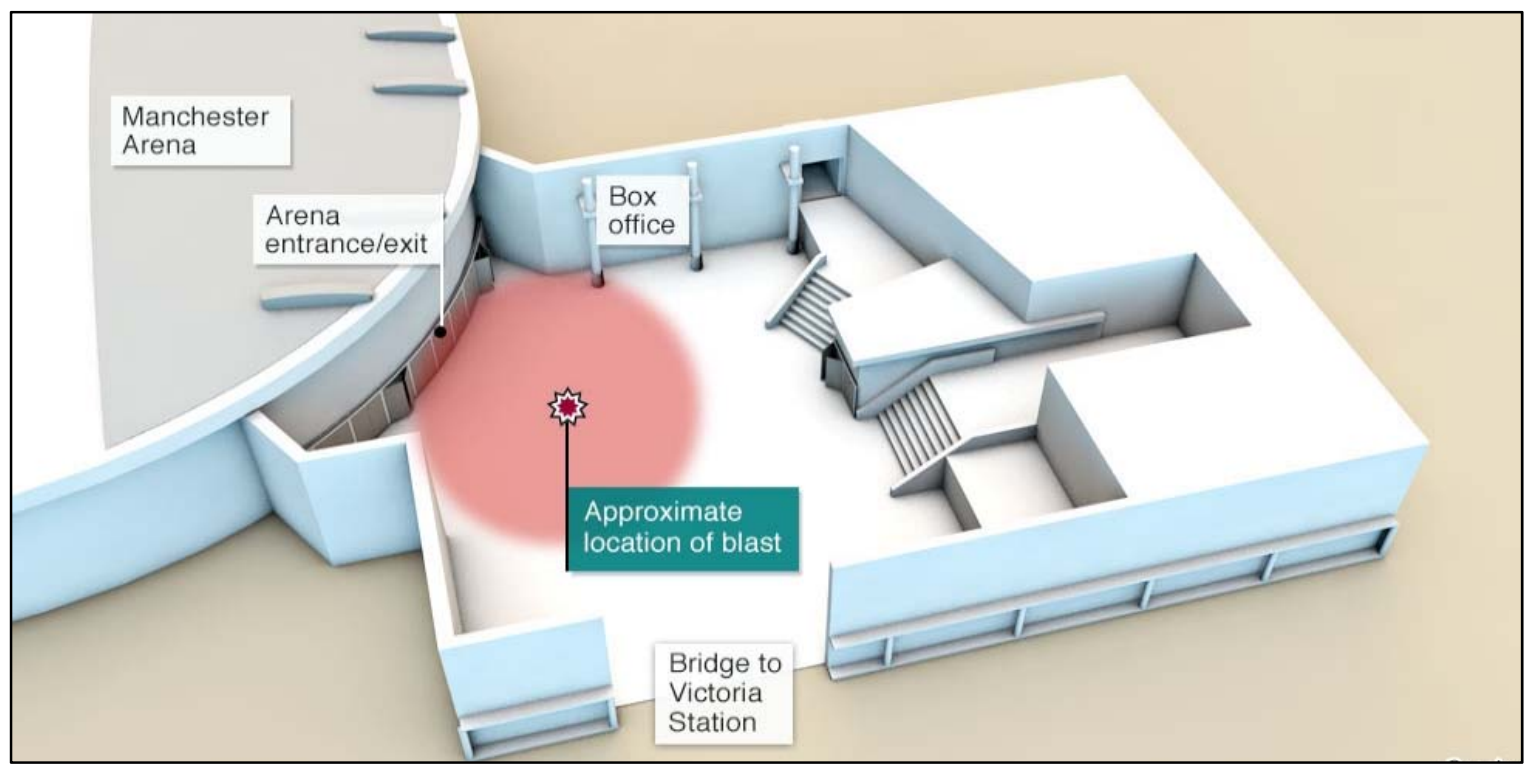

Figure 1.1 - An overview of the approximate location of the IED in the foyer of one of the venue's exits [1]. 
Shrapnel was embedded in people up to 20 meters away. Twenty-two people were killed. There was chaos inside the foyer, and people were covered in blood and bodies were strewn around the floor along with nuts and bolts with the scent of explosives. Shreds of a backpack, a battery and 'detonator' were later found at the scene-all that was left of the device. The Greater Manchester Police declared this terrorist incident the deadliest attack in the United Kingdom since the London Bombings of 2005.

This unfortunate terrorist attack involved the use of a bomb called an Improvised Explosive Device (IED) - IEDs are home-made devices constructed from military or other explosive material and deployed in unconventional ways resulting in a potentially deadly weapon. They are commonly used in terror tactics in a targeted population. IEDs range from

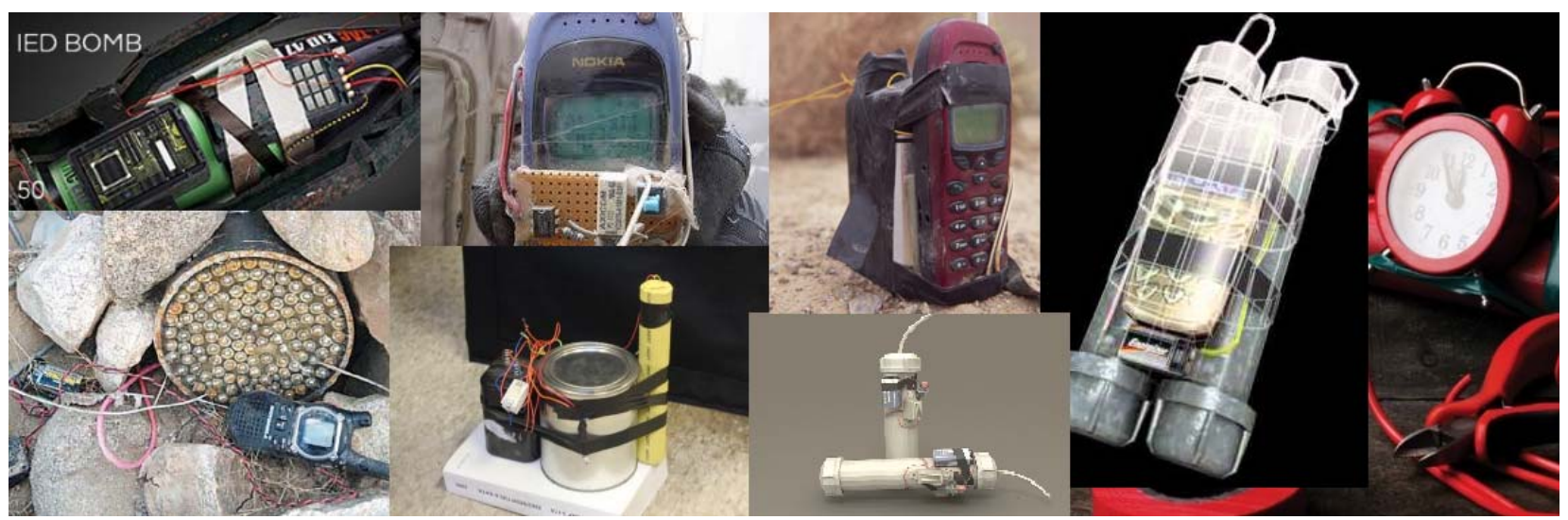

Figure 1.2 - Various IED contraptions, constructed in ways other than in conventional military action [2-6].

extremely rudimentary devices to sophisticated weapon systems containing high-grade explosives.

IEDs consist of several basic components, including an explosive charge and a method of detonation [19]. Explosives within an IED can vary from different types of dynamite, homemade explosives, high-grade explosives, and low-grade explosives. When the explosive component of an IED is concealed, such as inside an innocuous container, the type of explosive is impossible to 
determine through visual inspection. Thus, it becomes important to accurately surmise the type of explosive and determine the spatial location of key components by other means in order to dispose of the IED safely.

Specialized response teams - typically called "Explosive Disposal Units" (EDU), are public safety personnel that are trained in the safe handling of explosives and the threats posed by IEDs.

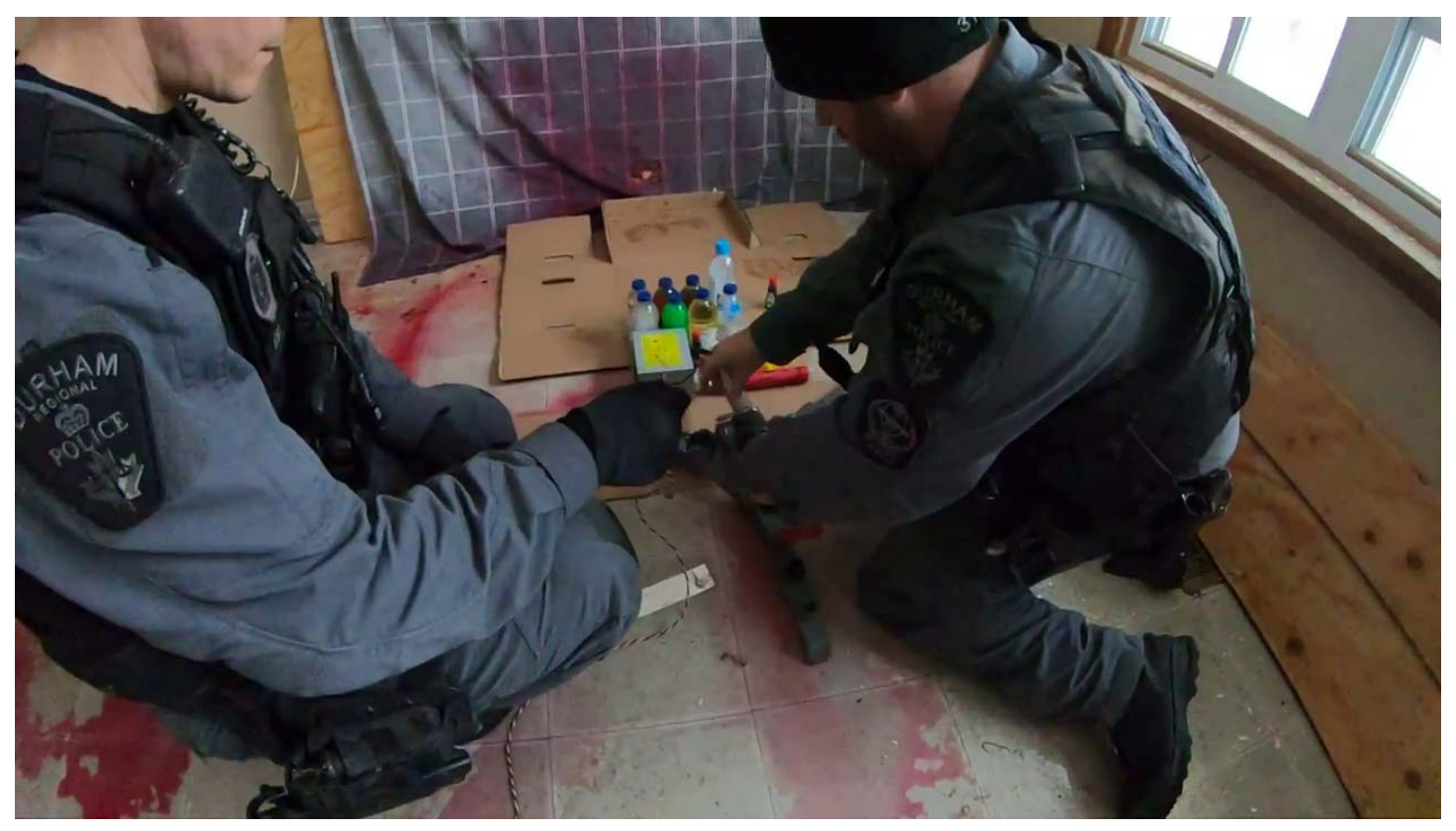

Figure 1.3 - An image depicting two EDU personnel positioning and arming a disrupter cannon.

EDU specialists use $x$-ray equipment [20] to screen and investigate the suspicious package $^{1}$. Based on the $2 \mathrm{D}$ image obtained from the $\mathrm{x}$-ray equipment, these specialists must estimate the spatial location of key components common in all IEDs; such as a main explosive charge, a method of detonation, a triggering device, a power source and shrapnel. Once these key

\footnotetext{
${ }^{1}$ The term "package" refers to a suspect IED hidden inside an enclosed container.
} 
components and its relative spatial location are identified, EDU specialists can make intelligent decisions for effective neutralization of the device.

IED neutralization involves using a liquid projectile (commonly water) fired from a highpowered dispersion weapon commonly known as a disrupter cannon. In the disruption process, the stream of liquid, propelled by an initiating charge, acts as a solid (penetrating the IED) and as a liquid stream causing dispersal of targeted components--removing key components of the IED without setting off the explosive charge by either rendering it inoperable or destroying it safely.

In this thesis, we build upon our previous work [21-23] that addressed this EDU neutralization decision making process. Our research in this area has culminated in the creation of a novel IED neutralization algorithm. We argue that this algorithm takes a significant step towards improving EDU neutralization processes. This algorithm consists of 6 methodologies or steps:

1. Estimate the pose, size and shape of occluded objects using 3D scanning technology.

2. Identify critical components of an IED of a 3D model.

3. Separate critical components of an IED of a 3D model.

4. Compute suggested possible neutralization aim points for a neutralizing shot by using disrupter pose and direction (Disrupter Pose and Direction methodology)

5. Compute relative densities of occluded objects (Relative Density methodology)

6. Compute spatial impact points from particle dispersion of components (Particle Dispersion methodology) 
With our novel algorithm, we hope to support EDU specialists with the task of accurately assessing future IED threat situations by providing them with additional technologies that incorporate various aspects of explosive handling and investigation. We hope to increase the tools available for safely analyzing real-world bombs by providing accurate and effective information to response personnel in a timely manner and enable them to make an informed decision based on the interpretation of that information.

\subsection{Problem Definition}

Suspect IEDs are assumed to be composed of unknown elements that are possibly volatile and dangerous. IEDs range in form, size and power and explosive components of an IED can be created using commonly available materials such as fertilizer and common chemicals [24]. IEDs can be created from a variety of explosive components that are a mixture of chemicals, or formed from conventional munitions like mortar and artillery rounds [4], [6], [19].

These IEDs can inflict harm in a number of ways; chemical-based IEDs can disperse toxic chemical agents that may spread unpredictably or fragmentation IEDs can explode and spread shrapnel comprised of bits of metal, nails and ball bearings in any direction [25].

There have been many examples of explosive charges constructed from everything from gun powder to fertilizer [26], [27]. Detonators (the part of the bomb that sets off the main explosive charge) can be as small as a $1 / 2$ in. in diameter. Triggering mechanisms (the timer or communication mechanism for the bomb) can be readily improvised through the use of a clock or cell phone and depend on the intended use of the IED, available resources and the knowledge of the builder. The 
power source (supplying electricity to the other components) can be made from common batteries [28].

Additional challenges to EDU teams arise when the placement of an IED is inside an innocuous container - these challenges are related to the fact that the spatial arrangement and the components of the IED are unknown. Each component has a separate mass, different density and may have varying states of matter (liquid, solid, gas, and/or aqueous). The explosive charge's power and effects are unknown and there is potential for secondary explosive threats (booby traps, or multiple explosives in the device). These challenges make it difficult to use solutions to model the power and effects of forces on objects that employ simple heuristics, such as flow topology from fluid dynamics that are designed to function only if objects are modeled in a closed system environment and attached to each other.

When specialized response teams deal with an IED threat, one of the guiding principles is to evacuate the people in the area, avoid contact with the threat, and determine the nature of the IED through safe and effective explosive handling. As mentioned previously, all IEDs consist of several basic components - main explosive charge, a method of detonation, a triggering device, a power source and shrapnel. These key components must be identified in order to effectively

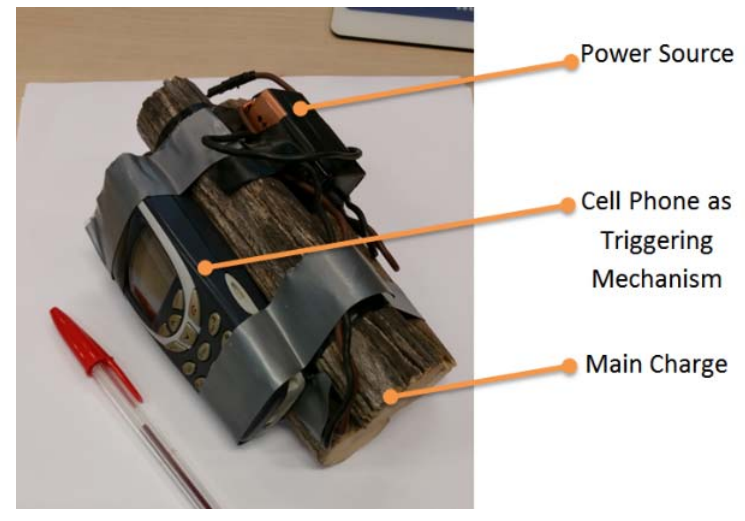

Figure 1.4 - Typical construction of an IED.

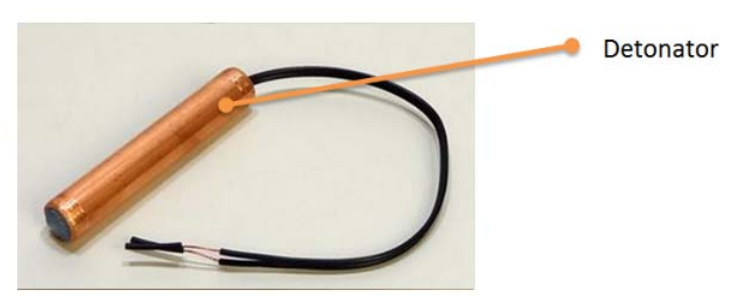


neutralize the IED as opposed to triggering an explosion.

EDU specialists use limited capability x-ray scanners and are equipped with various methods of image manipulation and enhancement tools that can help them identify the components in an IED. X-ray scanning procedures have many limitations, they include:

- the requirement of a clear "line of fire" between the x-ray scanner and the package, exposure to radiation and/or contamination by radioisotopes

- the requirement for manual tuning of the required energy charges (or penetration pulses) that are emitted from the scanner depending on visual estimates of the density of objects

- the requirement for proper positioning of the back-plate to capture projected $\mathrm{x}$-ray beams

- the need to replace the film and the scanner's bulb when overused

These limitations may increase the time an operator spends in the proximity of the IED thus endangering their own life.

In order for EDU to make intelligent decisions about how they should safely handle a particular explosive, they must address the key challenges of quickly scanning a suspected IED container and spatially identifying key components common in all IEDs and also determine primary and secondary effects (indirect object collisions as a result of the initial force of the object) of impact points on surrounding infrastructure as a result of neutralizing the IED.

In this thesis we argue that our algorithm addresses this decision making process by providing a number of solutions which are: 
- The creation of a 3D scan of the suspect IED and its interior components that allow us to estimate the pose, size, shape, and relative density of occluded objects,

- Creation of detached models of the suspect IED in a simulation,

- Computing possible neutralization aim points from the disrupter pose and direction information so that EDU specialists can practice IED neutralization, and finally

- Computing an accurate, open system, physics-based approach to component dispersion (particle dispersion) as a result of a high velocity projectile impact to model the primary and secondary effects of a disrupter shot.

This approach will also enable specialists with these tools to predict IED behavior based on 3D models and its unique characteristics of the IED within a game-based simulation. In doing so, we hope to support the accurate assessment of IED threat situations. 


\subsection{Objective}

One of our objectives was to create an algorithm that can be used to create a IED neutralization serious game simulation for the purposes of augmenting EDU processes.

In our initial approach, we provided a physically accurate representation of the IED that maintains all spatial relationships between all of its components and the container it is in and demonstrate reasoning based on information obtained from the game that mimics real-world characteristics and physics. We were able to manipulate those key components in a serious game which can provide objective scoring concerning the neutralization task. This approach allows operators to import arbitrary IED models into a game engine and reason about them.

Our next objective is to determine type of explosive employed in the device in order to properly plan an effective and safe means of neutralization. Since IEDs cannot be safely manipulated without endangering proximate people and property, it is important to find a method to ascertain to a degree of certainty the nature of the unknown materials in a concealed container and provide this additional information to EDU. We have proposed a methodology to calibrate a CT scanner and obtain a model to calculate relative densities as a form of additional information for threat-detection personnel. We argue that this methodology can be used to arrive at a reasoned estimate of the type of explosive being scanned as long as subsequent scans use the same predefined parameters, procedure and protocol and scanning machine.

Our final objective is to devise a solution to analyze shrapnel impact points and its effects on its surroundings. To this end, we have developed a shrapnel analysis simulation - this methodology suggests an open system game simulation may be useful in planning the disruption of real-world IEDs, in which game actors can position and target objects with a projectile of 
Newtonian characteristics such as user-defined velocity, acceleration, mass and density. This approach permits faithful reproduction of static and dynamic particle interactivity and may be tunable to emulate real-world effects without danger to the public or first responders.

This work stems from our previous findings [29] that enable us to determine relevant physical characteristics of unknown objects, with results that are obtained and verified from similar explosive analogs. The work was informed by our previous findings that enable us to compute and simulate spatial shrapnel impact points from a directionally focused charge calculated using flow topology algorithms.

We argue that this algorithm is relevant for EDU processes and can be accurately used to simulate particle dispersion, compute relevant major points of impact, as well as indicate areas of neutralization with a disrupter pose and direction, that may be useful in augmenting IED neutralization processes by using serious game techniques.

It is important to note that part of our overall algorithm focuses on developing the building blocks for creating 3D models using our developed generic methodology for relatively accurate scanning of inert explosives. Even though much emphasis is given to the use of CT scanning in this thesis, CT scanning is simply used in our methodology because it was an available resource. The main idea of our scanning methodology that we will describe is a relatively translatable methodology that may be applied to other threat detection scanning systems, not only CT scanners.

The implementation of the entirety of our developed algorithm for actual EDU processes are beyond the scope of this thesis, and only brief testimony from EDU personnel has been made regarding the efficacy of our work for their processes. Our algorithm acts as building blocks for 
creating relatively accurate 3D models of inert IEDs, and testing/implementation on actual realworld IEDs are not performed. 


\subsection{Contributions}

To the best of our knowledge, there currently exist no similar algorithm for developing simulations for EDU processes or in EDU practice that includes the following solutions:

1. Provide a method to visually inspect and separate 3D models of IEDs, and/or import arbitrary mock IED models and components into a game engine and reason about the IED system as a whole.

2. Determine the range of the type of explosive in an IED with relative densities.

3. Analyze particle dispersion as a result of a high velocity projectile on unknown occluded objects.

Therefore, our contributions are:

1. An augmented method for scanning simulated IED models using a 3D scanner.

Our current research augmented this scanning methodology by utilizing CT scanning technology to obtain DICOM data. This method produces high fidelity 3D virtual models and is capable of producing the following improvements:

1. Ability to capture more detail of metal objects - metallic compounds are known to cause distortion in scans that use magnetic-based technology for sensing.

2. Less virtual model pre-processing in terms of model cleanup. Model cleanup for our previous work involved removing excessive groups of artifacts in the virtual model due to metal artifacts resulting in inaccurate 3D virtual models.

3. The overall process of 3D virtual model creation using CT scanning technology produces high fidelity virtual models with more likeness in terms of its dimensions to its real-world analog (results are shown in Chapter 4). 
4. This method can possibly be applied to any scanner, as long as the same scanner is used throughout the system.

5. The expertise of an experienced technician is not a necessary requirement for performing calibration as long as the sequence of steps in this process is followed.

6. Other relevant objects that require a virtual model may be scanned using this process, for example, EDU equipment and EDU robots, given that the target objects are size appropriate for placement in a CT scanner.

2. An augmented method to create 3D virtual IED models.

Our current research augmented this method by cross-referencing each 3D virtual model with 3D virtual model obtained using Skannect. Skannect is a proprietary 3D scanning technology that creates a 3D virtual model using real-time stitching algorithms with data obtained from variations of handheld or stationary RGB-D cameras.

3. A method to determine a range of possible explosive types being employed in an IED that is hidden in a package.

Since IEDs cannot be safely manipulated without endangering proximate people and property, it is important for EDU specialists to obtain knowledge about the explosive to properly plan an effective and safe means of neutralization.

We have proposed a methodology to calibrate a CT scanner to ascertain to a degree of certainty the relative density of the unknown materials in a concealed container as a form of additional information for threat-detection personnel as long as subsequent scans use the same scanning machine and scanning parameters.

4. An augmented method to disintegrate 3D virtual IED components into parts (or particles) with specific characteristics of its real-world material: Each particle is programmed with 
characteristics of its real-world analog and subject to 3-dimensional physics when external forces are applied.

Our current research augmented this method and developed an ad-hoc algorithm that disintegrates the entire 3D virtual IED model into tiny parts (particles), and utilizes the information obtained in the data acquisition process in contribution \#1, that assists in producing the reasoning obtained from contribution $\# 3$ - to provide information such as density, size, mass, and pose that is associated with each particle. We add mutable variables such as velocity, acceleration and direction, which are used when calculating resulting forces on a particle when external forces from other particles are applied.

5. Demonstration of complex model interactions within serious game simulation and reasoning as a result of these complex model interactions.

Our current research adds to this reasoning by providing additional information on IED splatter impact points on its surroundings. We utilize the particle disintegration process from contribution \#4 that provides information such as density, size, mass, pose, along with mutable variables such as velocity, acceleration and direction which are all associated with each particle. This information is used to calculate primary and secondary impact points on nearby external surfaces. We claim that this contribution is relevant in understanding potential effects on nearby surfaces and suggest that the information is crucial to the IED neutralization planning process.

6. Particle dispersion as a result of a high velocity projectile impact methodology. We have devised a methodology to compute and simulate dispersion and collision effects of particles as a result of a high velocity projectile impact on a container with varying density particle models enclosed. Particle dispersion is computed using game engine 
physics, simple Newtonian mechanics as well as molecular dynamics on 3D scanned mock IED components which are disintegrated into particles. The simulation is validated with real-world experiments of a high velocity water projectile from various weapons including a disrupter cannon impacting on containers of liquid.

For clarity, contributions $\# 1, \# 2, \# 4$ and $\# 5$ are contributions that were already published in our previous work [21], [29], - and for this new algorithm, we have augmented/enhanced those contributions and supplemented it with contribution \#3 and \#6 (as published in our current work in [22], [23]) as a second part to this existing algorithm.

Contribution \#1 is augmented from our previous work which utilizes Magnetic Resonance Imaging (MRI) scanning technology to Computed Tomography (CT) scanning technology - in order to capture metallic elements and enhance image quality of scanned objects.

Contribution \#4 is augmented from our previous work that separates the virtual model into components (multi-part model), to a disintegration of the models into tiny particles (with each particle being associated with the component's material characteristics such as density) in order to suggest accurate calculation of the dispersion of IED components from a successful neutralization attempt using a disrupter cannon.

Contribution $\# 5$ is augmented as a result of the changes in contribution \#1, \#3 and \#4, in which the results of reasoning from neutralization attempts using a disrupter cannon, is outputted and can theoretically be used to position a disrupter cannon, and to estimate the potential effects of the components of an IED onto its surroundings after a successful neutralization attempt. 
Contribution \#5 also has some parts unchanged from our previous research work - in which component bounding boxes are associated in the same method as described previously, and it includes a scoring feature that provides users with feedback of their in-game performance which can also aid in suggesting desired actions from the user.

\subsection{Thesis Organization}

This chapter serves as an introduction to the following chapters. Chapter 2 reviews the literature and presents an overview of IED threats and EDU tactics, reviews 3D imaging technology and modeling techniques, reviews physics-based simulations and physics engines, and lastly, reviews high velocity projectile impact simulations and methods of effectively evaluating simulations to real-world effects.

Chapter 3 discusses our experimental design including the inert explosives, the various materials for density and mass measurements and the mock IEDs that were created and used and techniques used to collect our data. The methodology used to create 3D models from DICOM data along with Skannect data is presented, along with the methodology to determine relative densities of occluded objects. Next, we discuss the particle dispersion algorithm which disintegrates models into particles and models the particle dispersion of multiple occluded objects with varying density.

Chapter 4 discusses the experimental results of our relative density and particle dispersion methodology. For relative density measurements, we show and discuss density values obtained from scanning known substances and compare it to values from unknown substances. We show experimental results that compare our simulation to real-world experiments using a number of 
weapons capable of firing a controlled high velocity projectile onto containers of liquid with varying densities and masses. We test the points of impact (splatter) from the dispersion of realworld liquids of varying densities and masses to our simulation's dispersion of particles with these programmed characteristics. The degree of accuracy is evaluated with a sensor grid pad and splatter impact is visually estimated by the percentage of disrupted surface area. This is followed by an analysis and discussion.

Finally, Chapter 5 presents a summary of this thesis, the results that were obtained, limitations, and discusses some ideas for future research. 


\section{Chapter 2 Background}

The first section of this chapter will examine the literature related to IEDs in order to provide necessary background information to understand the entirety of the subject. A general introduction to IED threats--including a historical glimpse of high-profile IED-related incidents and their typical means of initiation-will be presented. This section is followed by a brief overview of EDU tactics and technologies that support current neutralization processes. The section will provide the reader with background information regarding the challenges being faced by first responders attempting to eliminate a threat to public safety by using current neutralization techniques on IED threats.

The following sections include a review of 2D and 3D imaging technology including $\mathrm{x}$-ray and computed tomography $(\mathrm{CT})$, along with a review of the data formats used by these imaging technologies and their conversion into 3D models. The section is intended to provide the reader with a better understanding of the limitations of these imaging technologies.

The contributions of this work are established and partially verified through a physicsbased simulation. The last section of this chapter includes a specific discussion of game engines in general and the Unity game engine specifically. It will include a discussion of popular physics engines such as NVIDIA FleX. As one of the contributions of this work relates to analyzing projectile impact points, this chapter also discusses high velocity projectile impact simulations and methods of effectively comparing simulations to real-world effects. 


\subsection{IED Threats}

IED threats can exist anywhere and they are evolving with new variants being regularly reported in the media. The use of chemical and explosive materials by criminals and terrorist groups pose a significant threat in every country, and may severely impact economic and political stability, endanger public safety, and increase the risk of further incidents (copy-cat) leading to an increase in the number of victims [30].

An IED threat inevitably results in a chaotic and hazardous environment requiring immediate response [31]. Each threat is assessed and appropriate resources to deal with the threat are assigned [2], [30]. Depending on the severity, threats to public safety may affect first responders at the local, regional, and national levels and may even require military resources.

The threat from IEDs has existed since the $16^{\text {th }}$ century, with a design that involves filling a hollow in the ground or rock with explosives (black powder) and projectiles. This design is known as a 'fougasse' weapon [32]. Variants exists such as stone, shell and flame fougasse, all of which have traits that, when fired, would scatter hazards to its surroundings, similar to improvised mortar, hand grenades and cluster bombs.

These weapons have been used in the American Revolution, as well as in Vietnam and with the British and Irish Republican Army. These weapons eventually led to more sophisticated designs such as "booby traps", "mines" and IEDs. The threat of IEDs has evolved since 2001 [30], and IED attacks have doubled from 2011 to 2014. In the first nine months of 2011, there were an average of 608 IED attacks per month in 99 different countries. For insurgents, IED are considered relatively cheap, and a relatively easy-to-use tool against civilians and military [33]. 
IEDs in Afghanistan are the major cause of explosive-related casualties among the general population [34]. Over the past few years, many different IEDs have emerged and new acronyms have been created to describe these evolving threats. They include vehicle-born IEDs, (VBIED), victim-operated IEDs (VOIED), command wire detonated IEDs (CWIED), suicide vehicle-borne IEDs (SVBIED), improvised rocket-assisted munitions (IRAM), and a growing number of other methods [34].

Because of an increasing number and diversity of IED threats, it is important to investigate additional preventative measures, that support counter-IED processes and techniques. Such an investigation might include detailed risk analysis, IED risk education, IED prevention and IED neutralization research.

Table 2.1 is a noncomprehensive list describing some high-profile historical IED-related incidents. A more detailed list of these incidents may be found in [35].

\begin{tabular}{|c|c|c|c|}
\hline Date & $\begin{array}{c}\text { Name of } \\
\text { Attack }\end{array}$ & Situation & Result \\
\hline $\begin{array}{l}1996 \\
\text { Olympics }\end{array}$ & $\begin{array}{l}\text { Atlanta } \\
\text { Olympic Park } \\
\text { Bombing }\end{array}$ & $\begin{array}{l}\text { In the midst of the } 1996 \text { Olympics, an IED } \\
\text { composed of "pipe bombs" concealed in a } \\
\text { backpack exploded in the Centennial } \\
\text { Olympic Park in Atlanta, Georgia, where } \\
\text { the games were being hosted. }\end{array}$ & $\begin{array}{l}\text { Killed } 2 \text { people } \\
\text { and many were } \\
\text { injured. }\end{array}$ \\
\hline $\begin{array}{l}\text { April 19, } \\
1995\end{array}$ & $\begin{array}{l}\text { Oklahoma } \\
\text { City } \\
\text { Bombing }\end{array}$ & $\begin{array}{l}\text { A truck bomb exploded in front of the } \\
\text { Alfred P. Murrah Federal Building in } \\
\text { Oklahoma City, Oklahoma. }\end{array}$ & $\begin{array}{ll}\text { Killed } & 169 \\
\text { people. } & \end{array}$ \\
\hline $\begin{array}{l}\text { March 11, } \\
2004\end{array}$ & $\begin{array}{l}\text { Madrid Train } \\
\text { Attacks }\end{array}$ & $\begin{array}{l}\text { Ten explosions rocked through four } \\
\text { commuter trains during rush hour on } \\
\text { March 11, 2004, in Madrid, Spain. }\end{array}$ & 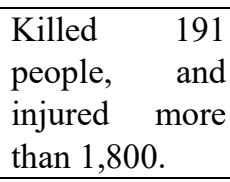 \\
\hline $\begin{array}{ll}\text { July } & 7, \\
2005\end{array}$ & $\begin{array}{l}\text { London } \\
\text { Bombings }\end{array}$ & $\begin{array}{l}\text { A series of coordinated attacks on the } \\
\text { London transportation system on the } \\
\text { morning of July } 7,2005 \text {. The attacks were } \\
\text { carried out by four suicide bombers. }\end{array}$ & $\begin{array}{lr}\text { Killed } & 52 \\
\text { people and } \\
\text { hundreds } \\
\text { injured. }\end{array}$ \\
\hline $\begin{array}{l}\text { July } 22, \\
2011\end{array}$ & $\begin{array}{l}\text { Norway Lone } \\
\text { Political } \\
\text { Extremist }\end{array}$ & $\begin{array}{l}\text { A lone political extremist bombed a } \\
\text { government center in Norway. }\end{array}$ & $\begin{array}{l}\text { Killed } \\
\text { people. }\end{array}$ \\
\hline
\end{tabular}




\begin{tabular}{|l|l|l|l|}
\hline $\begin{array}{l}\text { April 15, } \\
2013\end{array}$ & $\begin{array}{l}\text { Boston } \\
\text { Marathon } \\
\text { Bombing }\end{array}$ & $\begin{array}{l}\text { Two homemade bombs detonated 12 } \\
\text { seconds and 210 yards }(190 \mathrm{~m}) \text { apart at } \\
2: 49 \text { p.m., near the finish line of the annual } \\
\text { Boston Marathon. }\end{array}$ & $\begin{array}{l}\text { Killed 3 people } \\
\text { and injuring } \\
\text { several } \\
\text { hundred others. }\end{array}$ \\
\hline $\begin{array}{l}\text { September } \\
22,2013\end{array}$ & $\begin{array}{l}\text { Pakistan } \\
\text { Terrorist } \\
\text { Bombing }\end{array}$ & $\begin{array}{l}\text { Terrorists bombed a Christian church in } \\
\text { Peshawar, Pakistan. }\end{array}$ & $\begin{array}{l}\text { Killed at least } \\
78 \text { people. }\end{array}$ \\
\hline $\begin{array}{l}\text { November } \\
13,2015\end{array}$ & Paris Attacks & $\begin{array}{l}\text { A suicide bombing at the Stade de France } \\
\text { stadium were followed by more } \\
\text { explosions and shootings at popular bars } \\
\text { and restaurants in Paris. }\end{array}$ & $\begin{array}{l}\text { Killed } \\
\text { victims and } \\
\text { injured and } \\
\text { hundred of } \\
\text { others. }\end{array}$ \\
\hline $\begin{array}{l}\text { March 22, } \\
2016\end{array}$ & $\begin{array}{l}\text { Brussels } \\
\text { Bombings }\end{array}$ & $\begin{array}{l}\text { There were two suicide bombings at } \\
\text { Brussels Airport and another bombing at a } \\
\text { Metro station in the Belgium capital. } \\
\text { people and } \\
\text { wounded more } \\
\text { than 300 other }\end{array}$ \\
\hline $\begin{array}{l}\text { May 23. } \\
2017\end{array}$ & $\begin{array}{l}\text { Manchester } \\
\text { Bombing }\end{array}$ & $\begin{array}{l}\text { A lone suicide bomber detonated } \\
\text { explosives among teenage fans leaving } \\
\text { the concert at 10.33pm. }\end{array}$ & $\begin{array}{l}\text { Killed } \\
\text { people and } \\
\text { injured 59 } \\
\text { others. }\end{array}$ \\
\hline
\end{tabular}

Table 2.1 - A noncomprehensive list of IED attacks in different locations and different times.

\subsubsection{Improvised Explosive Devices}

IEDs are essentially "homemade" bombs intended to cause harm and/or damage to people and property when they explode. They vary in form, shape, size, complexity, and destructive capability.

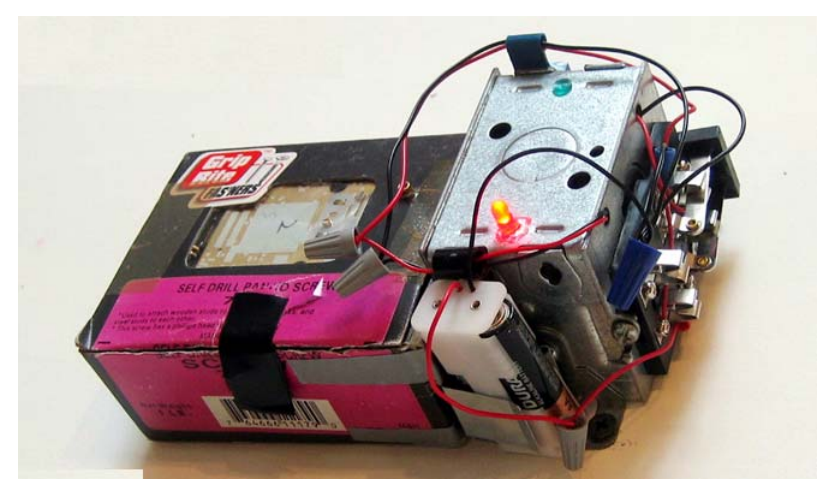

Figure 2.1 - An example of an armed Improvised Explosive Device [6]. 
As described in 2.1, the threat of IEDs are numerous, and IEDs have emerged as one of the weapons of choice for insurgents [3], [6]. These groups or individuals have resorted to the use of IEDs to advance a particular cause with varying degrees of effectiveness. Not only can an IEDs be placed inside innocuous containers, they can be embedded in vehicles, hidden under clothes on victims/perpetrators, wired remotely, attached to rocket-assisted munitions, or most recently, attached to unmanned aerial vehicles [23]. They can be made at low cost, are relatively easy to construct and place, and can achieve both strategic and tactical results, such as forcing troops to confine themselves to armored vehicles and travel at high rates of speed or plow through farmers' fields to avoid roads entirely.

IEDs have a long history, and the availability of surplus preprocessed materials have enabled the development and diversity of IEDs [6], [19]. Chemicals for explosives, and materials and products that can be modified to function as casings, shrapnel, triggering mechanisms and power sources have become readily available on a large scale.

The terms "booby trap", "mine" and IED are often used synonymously. An IED is essentially an explosive device that is fabricated in an improvised manner; incorporates destructive, lethal, noxious, pyrotechnic, or incendiary chemicals; and is designed to destroy incapacitate, harass or distract [30], [33], [34].

Regardless of design peculiarities, typically IEDs are composed of a main explosive charge, a method of detonation, a triggering mechanism, a power source and may include shrapnel. Table 2.2 provides a historical glimpse of IEDs and their typical means of initiation [34]. 


\begin{tabular}{|c|c|c|c|c|c|c|c|}
\hline Means of containment & & ypical & mean & s of In & itial & tion & Remarks \\
\hline & 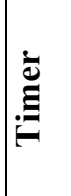 & 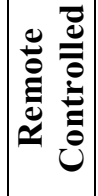 & ن & 焉 & 量 & 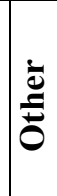 & \\
\hline Letter Bomb & & & & $\mathrm{X}$ & & & \\
\hline Pipe Bomb & $\mathrm{X}$ & & & & & & \\
\hline $\begin{array}{l}\text { Person-born IED } \\
\text { (PBIED) }\end{array}$ & & & & & $\mathrm{X}$ & & Suicide vest \\
\hline Mortar or Rocket & & & & & & $\mathrm{X}$ & $\begin{array}{l}\text { May be an improvised weapon or } \\
\text { factory made weapon using improvised } \\
\text { launcher }\end{array}$ \\
\hline Buried Bomb & & $\mathrm{X}$ & $\mathrm{X}$ & $\mathrm{X}$ & & & $\begin{array}{l}\text { Improvised mine when operated by } \\
\text { victim }\end{array}$ \\
\hline Box/Briefcase/Bag & $\mathrm{X}$ & $\mathrm{X}$ & $\mathrm{X}$ & $\mathrm{X}$ & & & $\begin{array}{l}\text { Includes typical roadside bomb, such as } \\
\text { palm oil container }\end{array}$ \\
\hline $\begin{array}{l}\text { Vehicle-Born IED } \\
\text { (VBIED) }\end{array}$ & $\mathrm{X}$ & $\mathrm{X}$ & $\mathrm{X}$ & $\mathrm{X}$ & $\mathrm{X}$ & $\mathrm{X}$ & $\begin{array}{l}\text { Could be divided into small, medium or } \\
\text { large }\end{array}$ \\
\hline
\end{tabular}

Table 2.2 - A noncomprehensive ID classification matrix of legacy IEDs and their typical means of initiation [30].

The table explores the range of possible improvisation of an IED's typical means of initiation and provides an overview of the different ways an IED can be embedded, which may aid in the determination of the appropriate allocation of resources for countermeasures to neutralize a threat.

The context of our work is any device which would normally be subject to a disruption such as an enclosed container for an IED (for example box, briefcase, or bag) or no container for an IED. These IEDs may include pipe bombs and letter bombs and their means of initiation (timer, or remote controlled).

The devices that cannot be disrupted are buried IEDs, person-born IEDs, IEDs attached to mortars or rockets or post IED-incidents. These instances may not involve EDU, may not warrant the use of a disrupter cannon and/or may be too late for EDU involvement. We focus on stationary 
IEDs (which may be enclosed in a container or not) that are possibly armed and not yet detonated - which pose a serious threat to public safety.

Within our scope, we explore examples of various types of explosive used in terrorist bombings [6] shown in Table 2.3.

\begin{tabular}{|c|c|c|c|}
\hline $\begin{array}{l}\text { Type of Explosive and Name of } \\
\text { Explosive }\end{array}$ & Common Uses & $\begin{array}{l}\text { Common } \\
\text { Form }\end{array}$ & $\begin{array}{l}\text { Known IED } \\
\text { Use }\end{array}$ \\
\hline \multicolumn{4}{|l|}{$\begin{array}{l}\text { High Explosives } \\
\end{array}$} \\
\hline 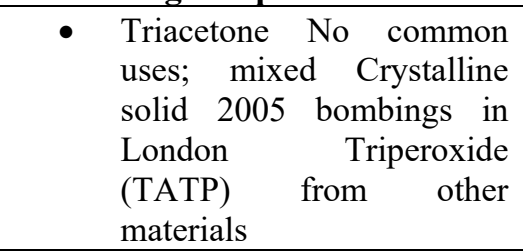 & $\begin{array}{l}\text { No common } \\
\text { uses; mixed } \\
\text { from other } \\
\text { materials }\end{array}$ & $\begin{array}{l}\text { Crystalline } \\
\text { solid }\end{array}$ & $\begin{array}{l}\text { London } \\
\text { Bombings } 2005\end{array}$ \\
\hline - GOMA-2 & Mining & $\begin{array}{l}\text { Gelatinous, } \\
\text { Nitroglycol- } \\
\text { based }\end{array}$ & $\begin{array}{l}\text { Madrid } \text { Train } \\
\text { Bombing } 2004\end{array}$ \\
\hline $\begin{array}{llr}\text { - } & \text { Semtex, C-4 } & \text { Primarily } \\
\text { military Plastic } & \text { solid Irish } \\
\text { Republican } & \text { Army } \\
\text { bombings } & \end{array}$ & $\begin{array}{l}\text { Primarily } \\
\text { military }\end{array}$ & Plastic solid & $\begin{array}{l}\text { Irish } \\
\text { Republican } \\
\text { Army } \\
\text { bombings }\end{array}$ \\
\hline $\begin{array}{l}\text { Ethylene glycol Component } \\
\text { of low- Liquid Millennium } \\
\text { Bomber, intended for } \\
\text { dinitrate (EGDN) freezing } \\
\text { dynamite Los Angeles } \\
\text { airport, 1999 } \\
\end{array}$ & $\begin{array}{l}\text { Component of } \\
\text { low-freezing } \\
\text { dynamite }\end{array}$ & Liquid & $\begin{array}{l}\text { Millennium } \\
\text { Bomber, } \\
\text { intended for } \\
\text { Los Angeles } \\
\text { airport } 1999\end{array}$ \\
\hline $\begin{array}{l}\text { - Ammonium nitrate Mining } \\
\text { and blasting Solid } \\
\text { Oklahoma City bombing } \\
\text { and fuel oil (ANFO) }\end{array}$ & $\begin{array}{l}\text { Mining and } \\
\text { blasting }\end{array}$ & Solid & $\begin{array}{l}\text { Oklahoma City } \\
\text { Bombing on the } \\
\text { Alfred P. P. } \\
\text { Murrah Federal } \\
\text { Building 1995 } \\
\end{array}$ \\
\hline $\begin{array}{lll}\text { - Urea nitrate } & \text { Fertilizer } \\
\text { Crystalline solid } & \text { World } \\
\text { Trade Center } 1993 & \\
\end{array}$ & Fertilizer & $\begin{array}{l}\text { Crystalline } \\
\text { solid }\end{array}$ & $\begin{array}{l}\text { World Trade } \\
\text { Center } 1993\end{array}$ \\
\hline \multicolumn{4}{|l|}{ Low Explosives } \\
\hline $\begin{array}{ll}\text { - } & \text { Smokeless powder } \\
\text { Ammunition Solid Olympic } \\
\text { Park bombings }\end{array}$ & Ammunition & Solid & $\begin{array}{l}\text { Olympic Park } \\
\text { Bombings }\end{array}$ \\
\hline - $\quad$ Black Powder & Ammunition & Solid & $\begin{array}{l}\text { Boston } \\
\text { Marathon } \\
\text { Bombing }\end{array}$ \\
\hline
\end{tabular}

Table 2.3 - Overview of types of explosives and their common uses, common form and the situation where such explosives were used [2]. 
The common form of the explosives listed in Table 2.3 demonstrate some of the target subjects of this research. EDU teams adjust steps in the neutralization process if knowledge of type of explosive, mass, and/or dimensions is known. It is ideal to surmise the possible range of the type of explosive as well as the density, mass and dimensions of occluded components in order to arrive at a reasoned approach to safe neutralization of an IED.

\subsubsection{Mine Warfare}

The term "improvised" also applies to the construction of the device by unauthorized or irregular forces (armed forces which are not authorized to use lethal or deadly force and weapons to support their own interests). A mine produced for regular armed forces may be considered an IED if it is used by irregular forces, whereas an unmodified mine placed by regular forces is not considered an IED. Explosive devices designed to disperse chemical, biological or radiological material are generally not classified as IEDs [36], [37].

As a precaution to exposing and providing detailed engineering schematics for IED construction, this section provides an overview of IEDs in mine warfare literature relating to the typical constructions of explosive-type IEDs. It will describe triggering mechanisms and initiators (igniters) but with insufficient detail to actually construct such a device.

In mine warfare, a typical explosive-type IED is normally operated externally by pulling on a wire, pressure, pressure release or release of tension on a wire or delayed action methods [7], [38$41]$. 


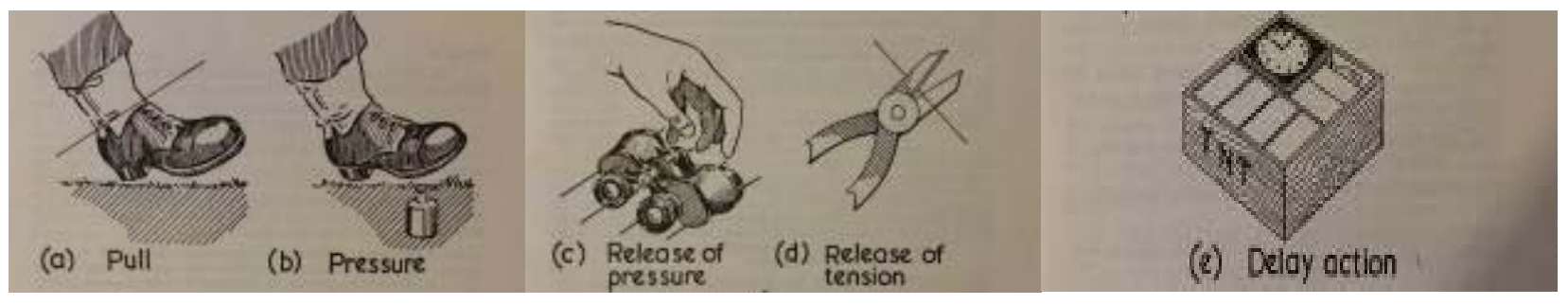

Figure 2.2 - Typical triggering mechanisms for explosive-type IEDs found in mine warfare [7], [38-41].

Internally, the mechanisms work along the same lines as mine igniters. The eight basic types of igniter are 1) spring operated, shear pin control, 2) spring operated, ball control, 3) spring operated, control by pin or plate withdrawal, 4) electric contact, 5) chemical electric reaction, 6) chemical reaction. 7) friction and 8) inertia [7], [38-41]. Figure 2.3 describes a switch IED using a pressure mechanism.

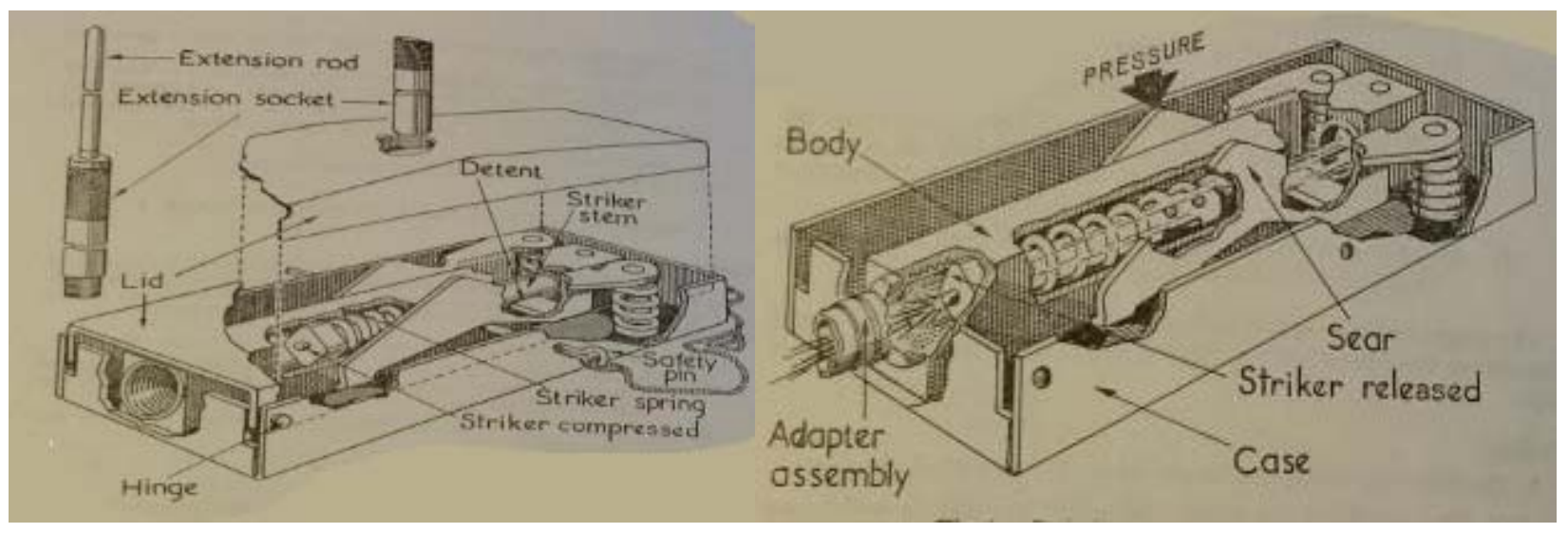

Figure 2.3 - Typical construction of a switch IED using a pressure mechanism [7], [38-41].

Spring operated devices are common [38]. They consist of a trigger device which, when set off, releases a striker, the striker fires a cap which initiates a fuse, and the fuse is led to a concealed charge. Figure 2.4 describes a standard method of connecting a mechanism to a charge. 


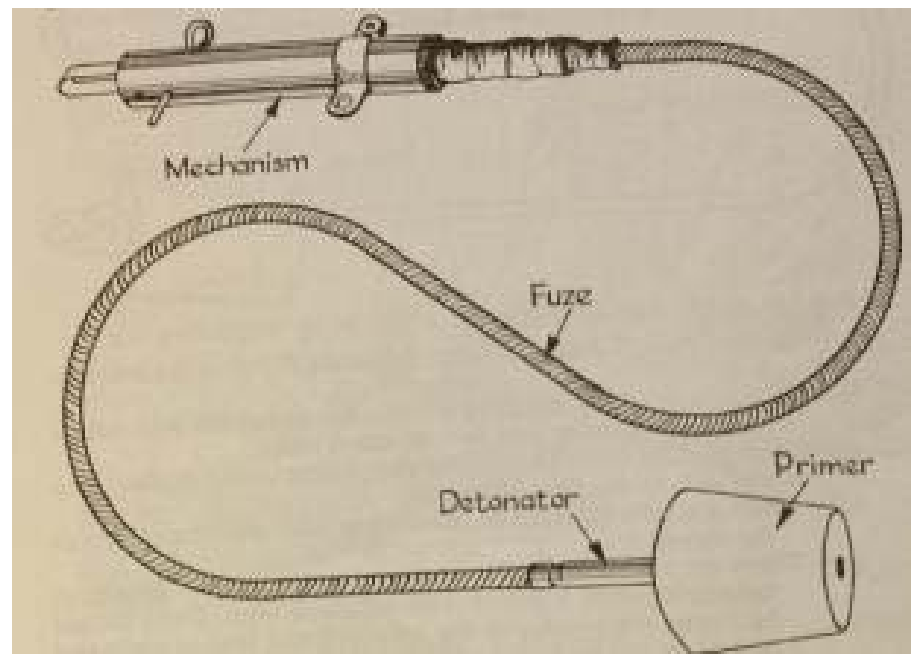

Figure 2.4 - A standard method of connecting a mechanism to a charge [7], [38-41].

Delay-action mechanisms can be fired by a variety of methods including clockwork, chemical reaction, radio and tension on a stretched piece of metal.

The principles governing the construction of IEDs are unlikely to change. However, the methods of setting IEDs in traps will vary with the ingenuity and skill of the operator, but it can be expected that IEDs will be designed according to their intended use.

Current counter-IED technology deals with relatively simple IEDs, and few advancements have been made to ensure countermeasures work for any IED anywhere [30], [31], [33], [42], [43]. Over $\$ 17$ billion USD was spent on IED-related countermeasures in 2011, resulting in a rising need for relatively cheap and scalable counter-IED solutions.

In the next section, we discuss EDU tactics and the reasoning behind the importance of obtaining a clear understanding of the IED as quickly and as safely as possible. 


\subsection{EDU Tactics}

EDU personnel are often unable to investigate a suspect IED without putting themselves at risk due to the uncontrollable nature and hazardous elements of an IED. IEDs can be hidden anywhere: on animals, planted in roads or strapped to a victim/perpetrator. They can be detonated via cell phones or trip wires, among other methods. They can be deployed in a combat environment or in the middle of a busy city. The adaptability of IEDs to almost any situation make it difficult to develop effective EDU tactics that work in all situations; however, there exist several common tactics to deal with IED threats.

\subsubsection{Explosives Disposal Unit}

The public safety task of identifying and neutralizing IEDs falls to military and police services with names similar to Explosive Disposal Units (EDU) who act to neutralize the threat associated with an IED, either rendering it inoperable or destroying it safely. EDUs train in various aspects of explosive handling and investigation which enable them to detect, identify, assess, render-safe, dismantle, transfer, and dispose of unexploded ordnance, improvised explosive devices and other explosive hazards. These operations also include decontaminating personnel and property exposed to hazardous materials during the response to a threat [44].

In explosives and ordnance disposal operations, there are three primary methods to neutralize an armed IED:

1) Neutralizing critical components. This involves scanning the device and visually determining critical components for neutralization (disarming components with a disrupter cannon). This process can involve x-ray scanning [45] to determine a critical 
but non-explosive component(s) of the IED and targeting the area in order to fire a liquid projectile causing dispersal of these components without setting off the explosive charge. The stream of liquid which acts as a solid can be windshield water fluid (methanol and ethylene glycol, to withstand a degree of freezing temperatures) and/or water [8], [46], [47].

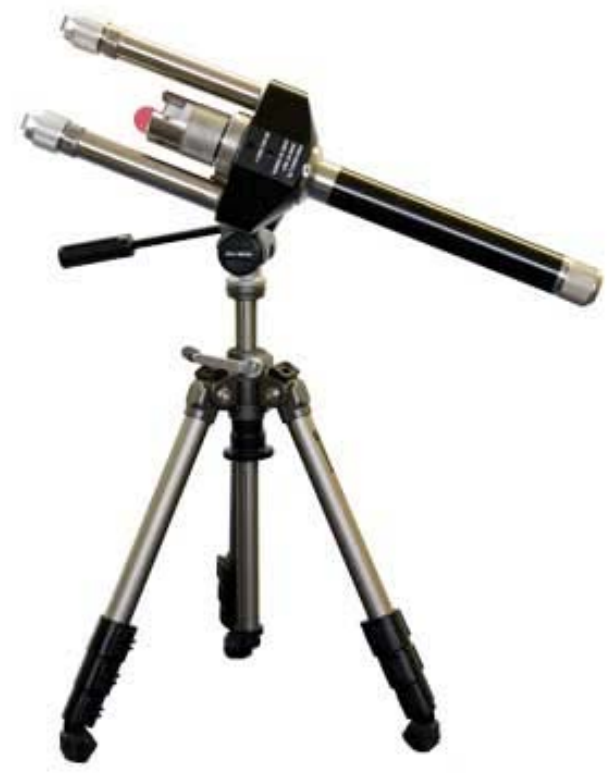

Figure 2.5 - A 20mm Recoilless MKIII Waterjet disrupter cannon by a defense supplier company [8].

1) Destroying the device in place. The other method is destroying the package with a prepared explosive charge using commercial explosives such as $\mathrm{C} 4$. This plastic explosive is placed at calculated locations around the bomb in order to simultaneously disrupt all components through the use of a controlled explosion. The use of C-4 explosives to deal with improvised explosives is considered a last resort in EDU operations, involves precise placement of explosive charges and is beyond the scope of this thesis. 
2) Placing the device in a containment vessel or chamber. Bomb containment vessels vary in size, shape, design, and its purpose can be to transport or to safely direct the explosion in a controlled manner. Total containment vessels can be large containers that can house large explosives and are designed to contain, transport and dispose of explosives or explosive devices. Partial containment vessels can be small containers that are designed to control the explosion of the bomb in a safe direction (usually upwards). These vessels and chambers are designed to withstand high-pressure blasts and adhere to standardized testing (ASME VIII and ASTM E.54.08).

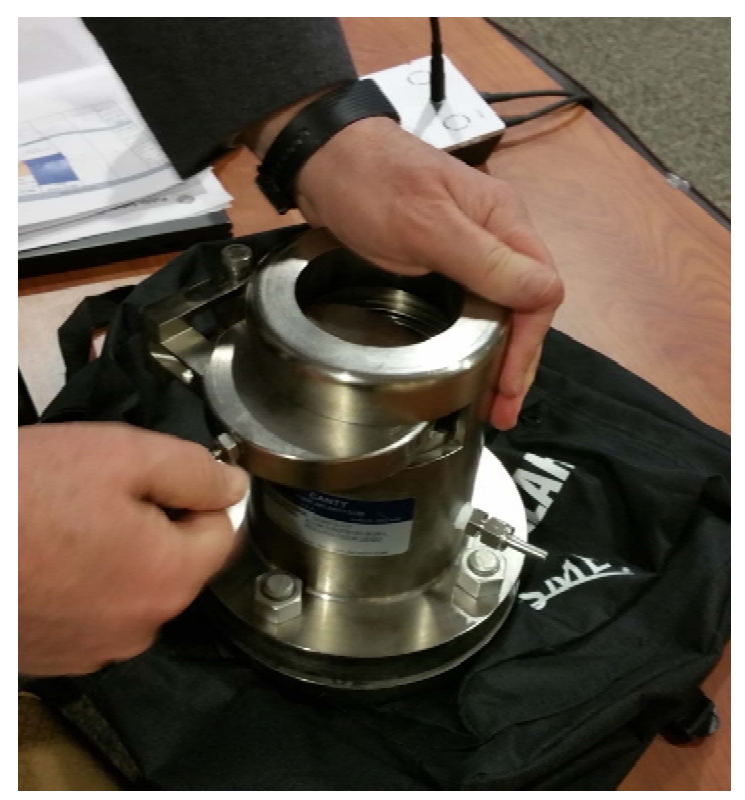

Figure 2.6 - A bomb containment vessel designed to direct an explosive blast upward.

\subsubsection{Disrupter Cannon}

The patent classification (F41B9/0046) describes disrupters - titled "neutralizing explosive devices". Patented inventions within this classification are various neutralization systems such as water cannons, liquid disrupters, interrupters, bomb circuit disrupting devices, or precision water jet delivery systems. Its function is to drive a liquid projectile into an explosive device such as a 
bomb (or IED)--powered by explosive or pneumatic propellant--with sufficient force to penetrate the casing that houses the bomb, and disrupt the detonation process so that the explosives are not triggered [9], [47-49].

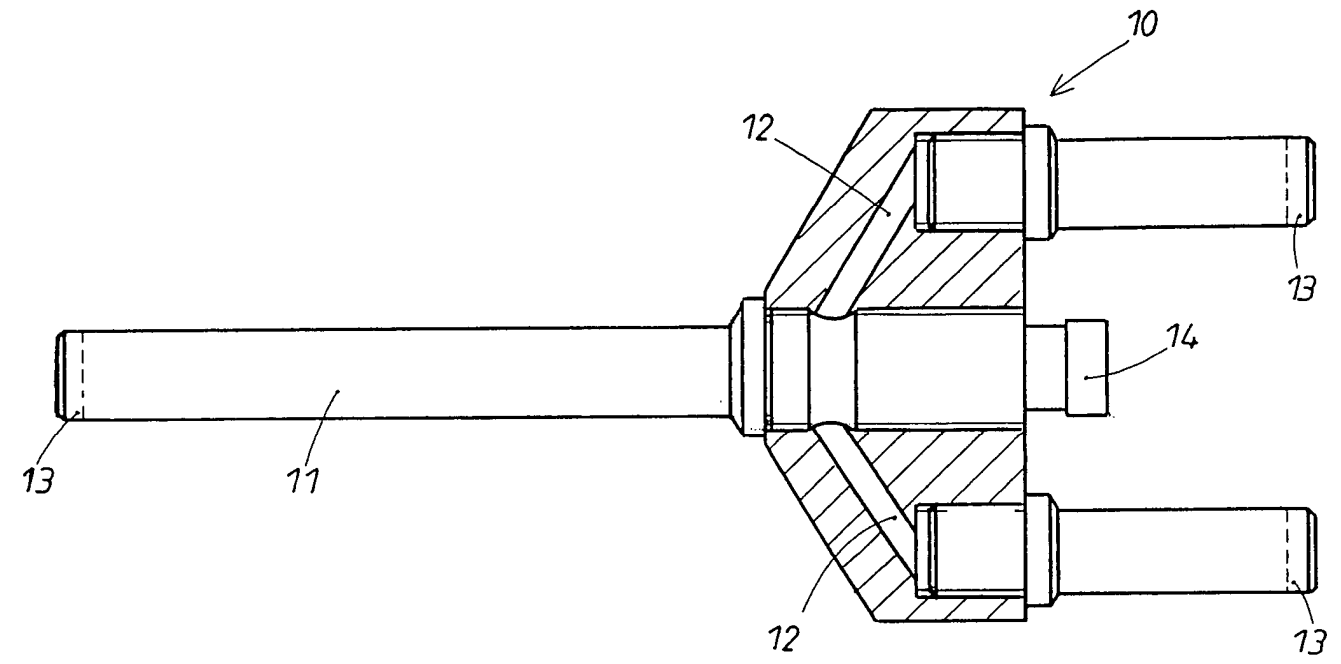

Figure 2.7 - The schematic for a device for disrupting IEDs, filed under patent “US7481146B2" [9].

The disrupter cannon in [9] works when the primer (located at the base of a cartridge containing propellant) is struck by a firing pin - this starts an initiation process which accelerates the projectile and propels it through a barrel.

For the projectile, a water projectile or a plaster slug can be used and provides for a relatively low-energy destruction of bomb components. From past examples [47-49], water used as a projectile has proven to be an especially effective means of neutralization. A commercially available cartridge can be filled with the water and then guided into the disrupter to ensure that the projectile keeps its shape for a longer period of time after the acceleration. This cartridge increases the effectiveness of the projectile. If the device is used at temperatures below 0 degrees Celsius, antifreeze solutions can be added to the water projectile. 
The barrel of a typical disrupter is 2 to 3 feet in length, and is a hollow cylindrical tube which is constructed of high grade stainless steel, that is sealed at its rear and sealable at its front. The chamber of this tube is capable of withstanding pressures of 20,000 psi. The primer, propellant cartridge and projectile cartridge is loaded inside the barrel to the rear (breach), and the front end of the barrel is closed with a water-tight seal - the seal is for safe transportation and holding the cartridges in place in the barrel. The firing pin has a safety lock pin which is not removed until the disrupter is positioned and directed at the target and an explosive technician verifies that the area is clear of people and that the firing sequence can safely begin. Liquid water (or anti-freeze) is used as it is nearly incompressible and water typically cause electronics to short circuit and malfunction when in contact.

Disrupter cannons have a range of support mounts and can be adjusted to target a device in any position given sufficient room. Upon the charge exploding in the barrel, the projectile is propelled out of the barrel at high velocity (which can reach the speed of sound), rupturing the frangible seal closing the opened front end of the barrel. EDU specialists must position the cannon to account for secondary effects from the disruption process. The intent of firing the projectile is that it should penetrate objects at such a high velocity that any tampering detection systems within the IED do not have enough time to react and prematurely detonate the bomb.

Depending on the perceived thickness of the surface of the container housing the IED, EDU teams adjust the power of the cannon by loading a propellant cartridge containing more or less propellant to change the velocity of the projectile [50]. 


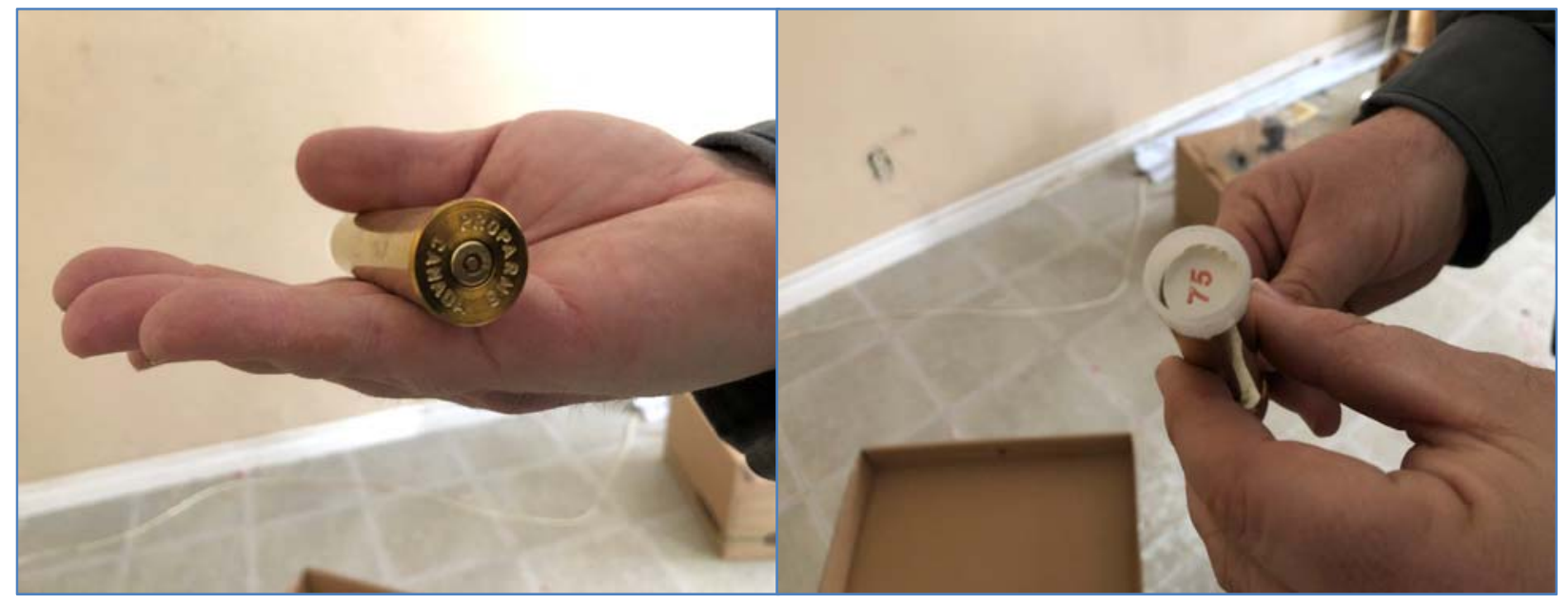

Figure 2.8 - A shell casing of the propellant (left) and a number depicting the charge of the propellant (right).

\subsubsection{EDU Scanning Equipment}

The method of scanning a bomb and disrupting components within, depend on visually determining regions of an IED that may resemble any one of the key non-explosive components (power source, triggering mechanism, detonator), for the purposes of IED neutralization. The $\mathrm{x}$ ray scanner directs a pulse of electromagnetic radiation (x-ray) at the IED which is, in turn, projected onto a film inside a black-plate, similar to how light is projected through a pinhole onto film in a camera. The x-ray scanning process of IEDs allows the sensing of internal components through the varying degree of $\mathrm{x}$-ray absorption in each component and the intensity of $\mathrm{x}$-rays that penetrate/diffract/reflect those components and reach the film. Using currently available technology, each scan will produce one $2 \mathrm{D}$ image with no depth differentiation, and subsequent scans require replacing the film in the black-plate. 


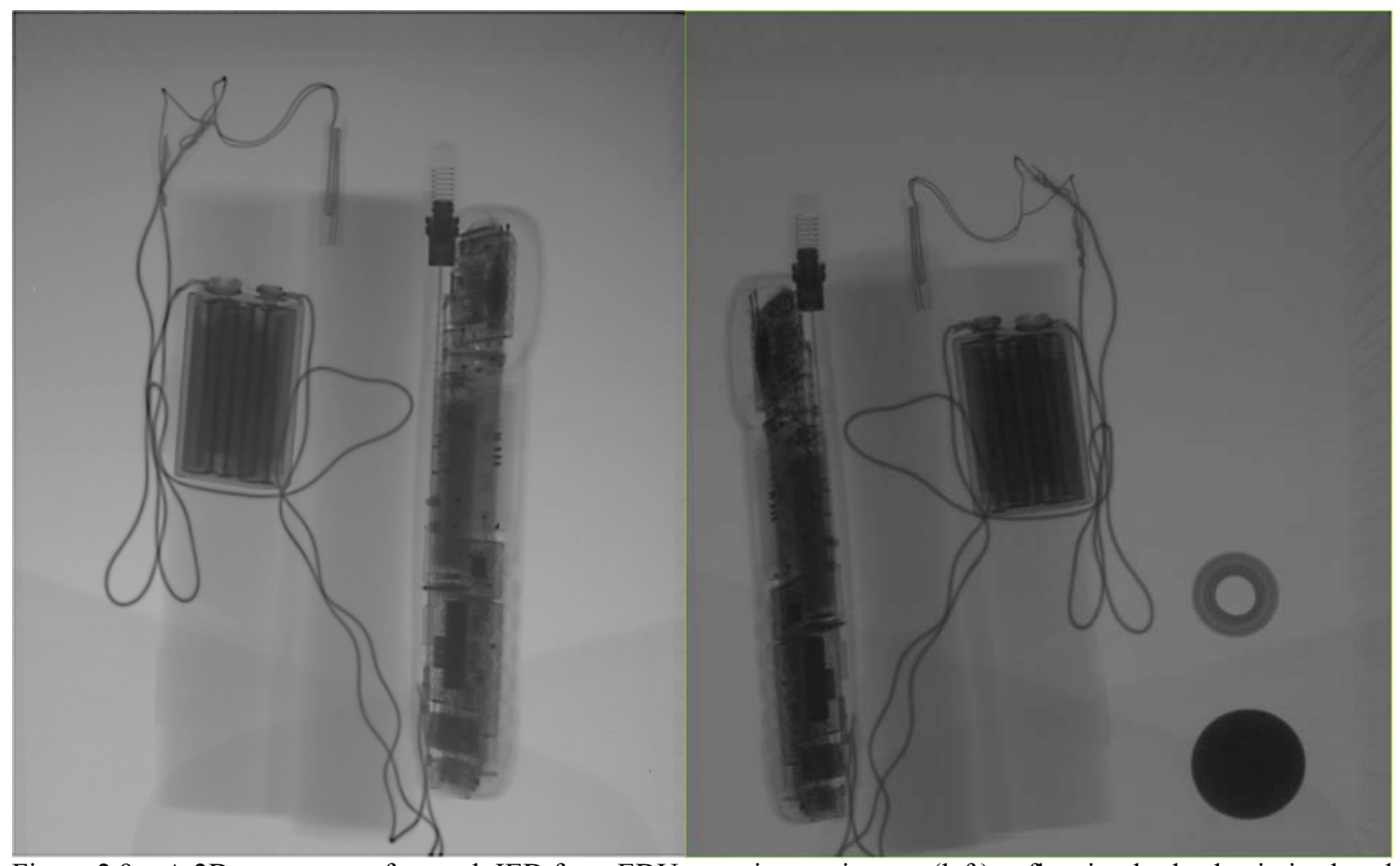

Figure 2.9 - A 2D x-ray scan of a mock IED from EDU scanning equipment (left), a flat circular lead coin is placed beside the scan for reference, the darker the intensity of the image, the denser the material (right).

Based on the 2D image, EDU personnel can estimate regions where actual components may be located, and plan to aim a disrupter at the presumed location of the component to neutralize the IED. The use of x-ray imaging to identify IED components is similar to medical radiographs for identifying bone structures, in which bones containing calcium absorbs x-rays and reduce the amount of x-ray reaching the detector in the shadow of the bones, making it visible on the radiograph. Trapped gasses also show up clearly because they absorb fewer x-rays compared to bones. Detailed information relating to x-ray technology will be explained in section 2.4 "Imaging Techniques for Threat Objects".

However, unlike medical imaging x-ray processes, EDU scanners emit high powered x-ray photons that carry enough energy to ionize atoms and disrupt molecular bonds. This type of 
ionizing radiation can therefore be harmful to living tissue, and each burst of $\mathrm{x}$-ray photons at a high intensity may overcharge and cause overuse of the x-ray emitter bulb which is a secondary problem.

When using the x-ray technology only, the potential number, variety and complexity of IED designs can make it difficult for EDU personnel to safely investigate a bomb's characteristics and effectively neutralize its threat without endangering themselves.

\subsection{Medical Imaging}

Medical imaging is the process of creating visual representations of the interior of a body for clinical analysis [51]. For example, X-rays are one form of medical imaging producing 2D, black and white representations of internal structures. The primary use of medical imaging is to reveal internal structures hidden by skin, muscles and bones, as well as to diagnose and treat certain diseases. Decades of research has been applied to technologies related to medical imaging leading to its adaptation for use in other fields such as threat-detection, Urban Search and Rescue (USAR) sensing, industrial engineering sensing within vehicle manufacturing, and public security applications such as airport cargo/luggage scanning [52].

\subsubsection{X-Radiation Imaging Systems}

There is significant widespread use of x-ray imaging systems in security screening applications [53] such as screening for threats in airport security, freight containers, border patrol and high-profile government buildings. In airport security, screening systems are tasked with producing a series of 2D images of the contents of luggage/cargo that is suitable for both visual 
inspection and for analysis by automatic threat detection algorithms. This process provides operators a tool to check luggage without opening and manually checking each item.

$\mathrm{X}$-radiation, or x-rays, is a form of electromagnetic radiation, with wavelengths ranging from 0.01 to 10 nanometers, and energies in the range $100 \mathrm{eV}$ to $100 \mathrm{keV}$ (electronvolt) [54]. Xrays can be divided into two categories; soft x-rays, (photon energies below $5 \mathrm{keV}$ ) and hard $\mathrm{x}$ rays (photon energies above 5-10 keV) [55]. The higher the energy, the better the penetration of dense objects, but drawbacks are that it requires an expensive emitter (accelerator) and detector, requires more time than low energy pulses per scan, and requires special cooling systems for the accelerator per scan. However, with low energy x-rays, it may not penetrate thick metal walls, heavy machinery and other metal objects. In the scanning task for freight containers and large transport vehicles, there exist the use of high energy x-ray systems that emit x-rays in the range of mega-electronvolt $(\mathrm{MeV})$ and imagers suitable for efficient detection of high energy x-rays [55].

When target objects are passed through an x-ray scanner, radiation is passed through the object and a detector senses variations in the intensity of the radiation exiting the object. The detection process can be categorized into diffraction (measurement of the precise angles and intensities at which x-rays diffract from the object) and backscatter (measurement of the intensity of radiation that reflects from the object) - both of which forms an image.

The x-ray absorption depends primarily on the material's density, which means that the higher the density is, the higher the energy absorption by the object, and hence the darker the image. This process uses the differential absorption law [56] to form a 'projection' of the materials, which appears as an image of the interior of objects. 
To address the problem of discriminating between different materials with overlapping, or superimposed objects in a 2D image, there exist techniques to view complex objects by dual energy imaging (fusing low and high energy images) [57], and multi-view x-ray imaging [58]. Figure 2.10 depicts a chart of particular $\mathrm{x}$-rays in the electromagnetic spectrum.

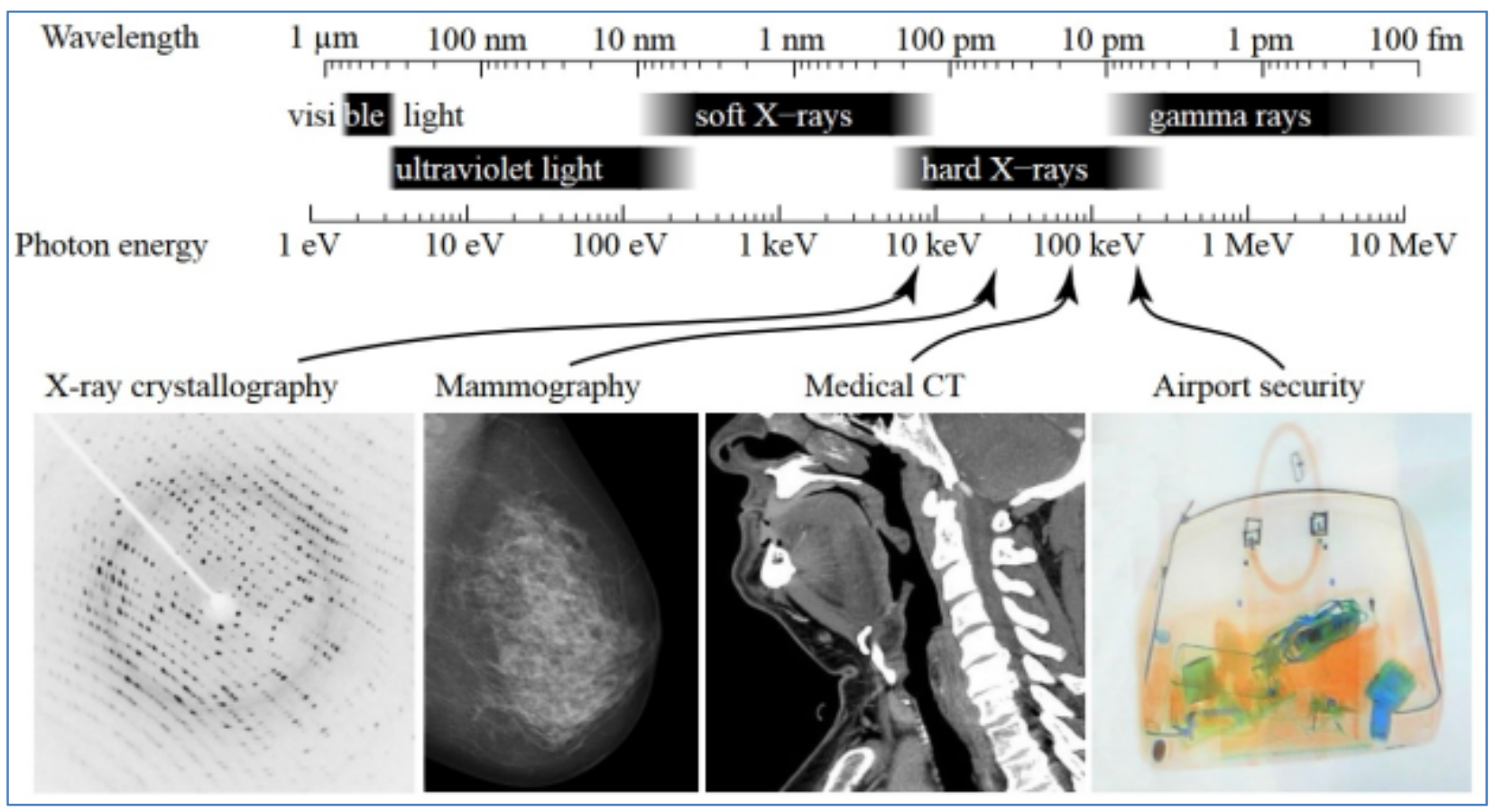

Figure 2.10 - A diagram depicting different applications that use different parts of the x-ray spectrum [10].

These x-ray imaging techniques to detect threat objects still suffer from a number of limitations, such as:

1. Loss of Generality - approaches that are developed ad-hoc for one application and may not be transferred to another.

2. Deficient Detection Accuracy - A fundamental tradeoff between false alarms and miss detections.

3. Limited Robustness - Scanning process is meant for simple structures only. 
4. Low Adaptiveness - Difficult to accommodate an automated system to account for additional modifications or different scanning targets.

Numerous research works in image recognition algorithms and enhanced x-ray sensors have been developed for x-ray systems to better sense and automate (partially) the identification of objects of interest inside a complex object [57], [59], [60]. These systems often require the involvement of a human operator in the final stages of the object recognition task for additional inspection and often have limited technological support. These systems greatly rely on operator experience and expertise to effectively reduce the risk of (or deter) crime and terrorist activity. As a result, human operators for security screening systems may suffer from a) demanding and stressful process (limited window of time for inspection during peak hours at airports) b) arduous and tedious task (sustained focus and attention to discern threatening articles) c) a level of difficulty (operators undergo object threat detection programs and receive minimal technological support) d) a degree of uncertainty (possible occurrences of human error/oversight/negligence/neglect) [61].

EDU are trained to operate x-ray screening instruments that rely on the operator's ability to visually identify potential threats by interpreting $2 \mathrm{D} x$-ray images. The efficacy of these threatdetection screening systems are highly reliant on the interpretation of the data by the personnel who operate them. An alternative to 2D x-ray images is Computed Tomography (CT).

\subsubsection{Computed Tomography}

Computed tomography, also called x-ray computed tomography (x-ray CT), is a scanning technology that is a commonly used form of medical imaging for viewing multiple cross-sectional 
(tomographic) scans of internal body structures, and can aid in determining the location, size, and relative densities of unknown masses.

With the use of CT scanning for detecting threat objects, an operator can view a container's contents in 3 dimensions without physically interacting with it. It also provides the benefit of creating 3D reconstructions and multi-planar views that allow the detection of unknown masses including allowing the determination of their locations, sizes and relative densities with respect to surrounding materials.

CT is simply a combination of many x-ray images taken from different angles to produce cross-sectional (tomographic) images, or virtual slices [11]. These slices contain information about the inside of an object, or rather, volume data, and require a lot more storage space than just surface data. Surface data on the other hand, is usually represented as polygonal meshes made up of vertices, edges and faces representing only the outer contours (essentially the surface) of an object. 


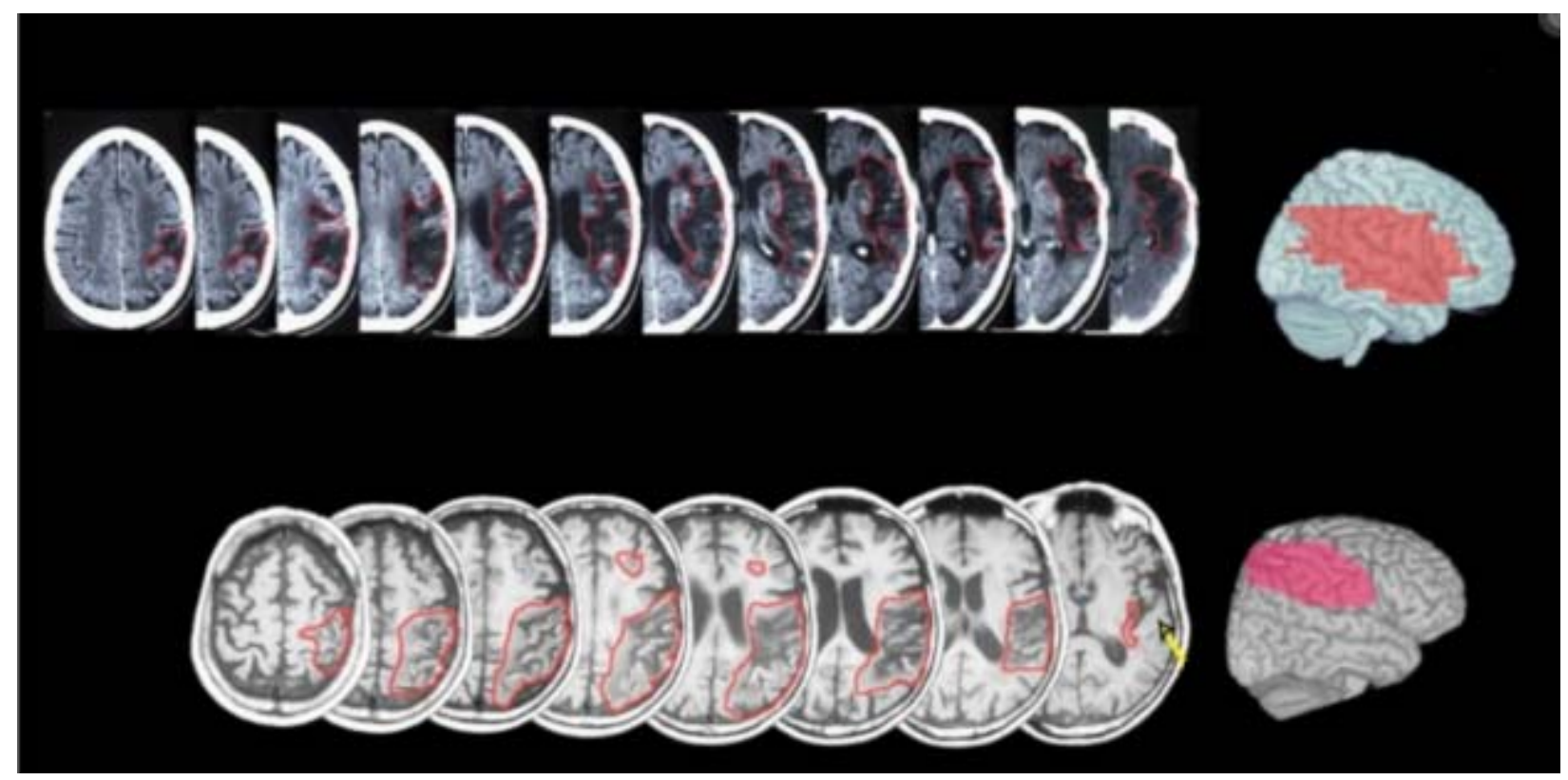

Figure 2.11 - Image slices of a brain from a MRI scan, similar to a CT scan [11].

In CT scans, the colour of a pixel in each image slice represents the density of the material at that position, measured in Hounsfield units. In medical images, usually, bone structures would have the highest density (around 700-3000 Hounsfield units (HU) [62] and therefore its value would be highest or closest to white), whereas tissue would have a lower density at 100-300 HU, followed by muscle and blood at 10-45 HU. Air would likely have the lowest density represented by a colour closest to black.

The size and density of the target are increasingly problematic. For instance, a larger and denser target would require more x-rays and larger field of view, whereas a smaller and less denser target would require less x-rays and a smaller field of view [63]. In order to obtain relevant information from CT scans, it is essential for operators to have thorough knowledge and practical experience with CT scanning technical parameters, standard procedures and protocols — none of which are current practice for EDU. 
It is important to note that results obtained from scanner to scanner vary significantly, even when the same brand and model of scanner is used. Other variables that may affect the internally generated raw data values may be scanner parameters, reconstruction artifacts or the use of different scan geometries [64]. Levi et al. describes that internally generated raw data values from CT scanners are unreliable as absolute values and caution is advised in the use of these values for diagnostic and therapeutic purposes [64].

However, it is possible to obtain values that can be associated with specific parameters and a particular scanner, enabling a relative density measurement. This measurement can be relevant as long as the same scanner and parameters are used to scan subsequent targets.

Utilizing CT scanning technology as a method of scanning IEDs has many potential advantages for EDU personnel over the current $\mathrm{x}$-ray methodology as it allows EDU to see the interior of an enclosed object with precision. In addition, actual scans take less than 30 seconds and an operator can change the imaging plane without moving the object being scanned.

CT scanning technology is not without challenges. CT scanners tend to be large, exceedingly expensive [22], [55], and may not be easily transported to the site of an incident. There is considerably more expertise required of the operator in addition to the challenges of recalibration of parameters, and there are the challenges of positioning the CT scanner in order to image the target IED without disturbing it.

For the moment, CT imaging systems cannot readily be used for many EDU operations and are beyond the capabilities of most EDUs--who have to make due with x-ray equipment. However, until recently, a range of components of different sizes, shapes and forms can exist 
within the target being scanned which can allow for the scanning of mock IEDs to create virtual IEDs for a simulation, and portable CT imaging systems are now employed in military applications.

\subsubsection{Imaging Techniques for Threat Objects}

In terms of airport security applications, a number of practical problems present a set of challenges to imaging technologies for cargo/luggage inspection- which include detection and inspection of complex objects [58], and overlapping objects in a single view.

Applications for object threat-detection include efficient and effective automated detection systems [65], use of modern computer vision techniques for automated object recognition [66], and image reconstruction/enhancement techniques [53], [55], [57], [59-61], [67]. Airport cargo security at peak times often have to scan over 1500 individual pieces of luggage per hour, which provides only a limited time frame to check for threats per unit of cargo.

Other common explosive and related threat-detection methods include technologies such as walk-through metal detectors (WTMD), handheld metal detector (HHMD), explosive trace detectors (ETD) and stationary and portable x-ray technology [68]. Remote detection systems containing intensified charge-coupled device (ICCD) detectors have also been designed and demonstrated for possibly detecting high explosive materials [69-71] or other chemicals [72]. The use of chemical detectors (swabs and canines) are also used to detect potentially dangerous chemicals which are outside the scope of medical imaging and will not localize the threat (where exactly is the bomb, but potentially shows the existence of chemicals). 
Our focus is the detection and localization of concealed explosives in containers. IEDs, which cannot be safely manipulated without endangering proximate people and property. Common EDU practices use x-ray technology but do not use WTMD or ETD threat-detection technologies when dealing with a suspect IED [24], [73] as specialists cannot physically interact with the target (ETD requires direct contact with the target for explosive trace analysis and WTMD requires the target to be moved), therefore our focus is on remote non-invasive and non-destructive (low to medium energy) x-ray imaging systems.

\subsubsection{Other Imaging Systems}

There are numerous other imaging systems that could conceivably detect concealed explosives in containers, for example, ultra-high frequency scanners, neutron scatter camera, binary detection system, colourmetric test kits, canines, honey bees, mechanical scent detection, spectrometry, neutron activation, and last but not least, silicon nanowires as field effect transistors [74]. These detection systems are out of the scope of this thesis, however future work may involve the implementation of these systems in our generic scanning methodology.

Although our scanning methodology focuses heavily on the use of CT scanners, our intention is to have a relatively adaptable generic scanning methodology that may be portable for implementation on other imaging systems. The implementation of our proposed scanning methodology on other imaging systems is beyond the scope of this thesis.

\subsection{Three-Dimensional Model Reconstruction}

There are numerous methods, algorithms and techniques for reconstructing multiple images into a three-dimensional model. Some methods require extra metadata such as depth data, 
and some methods triangulate a position of a 3D point from the intersection of two projected rays from two images. Many of these methods require special cameras, and/or pre and post processing of multiple sets of 2D images. Whatever the technique, they all synthesize information that pertains to the $3 \mathrm{D}$ structures in the $2 \mathrm{D}$ data in relation to their pose and the calibration of the optical sensor.

\subsubsection{DICOM Data}

Before we proceed with a discussion of 3D modelling, we will briefly discuss the Digital Imaging and Communications in Medicine (DICOM) format which is a standard for handling, storing, printing, and transmitting information in medical imaging. It also includes a communication protocol designed to transmit patient data across networks. DICOM data is an ISO standard widely known for its workflow and data management. It is the standard format for capturing data obtained from CT scanners. A CT machine captures DICOM data on three hypothetical planes which transect the target; they are called the axial, sagittal and coronal planes.

\subsubsection{D Model Reconstruction using DICOM Data}

The $3 \mathrm{D}$ reconstruction using DICOM data depend on the scanning method and the output parameters used - they contain data that is digitized information of the tomographic images which are obtained through the measurement of varying radiodensities of matter. There are many applications that provide simple 3D reconstructions of DICOM data; 3D Slicer, 3D DICOM by Sante, ImageJ, Amira, Mimics, ITK-snap, Meshforge, Osirix [75], [76], among many other volume rendering software [77]. Volume rendering techniques for DICOM range from segmentation algorithms, such as watershed segmentation algorithm, to superimposition (placing an image on top of an already existing image) and analysis algorithms, such as the least-squares registration algorithm (to align similar landmarks of superimposed tomographic images) [78]. 
DICOM file formats store a number of metadata (date, time, protocols, sequence type, image dimensions, bits allocated etc...), however, for the scope of our work, we are only concerned with the field of view, view angle, slice thickness, slice spacing, tube current, type of sequence, resolution and the total number of voxels (voxels represent the smallest distinguishable box-shaped part of a 3D space).

These parameters are adjusted most frequently and affect the quality of the scans and time it takes to create a set of DICOM images according to a CT technician [79].

\subsubsection{D Model Reconstruction using Multiple Images}

Many techniques exist that utilize multiple imagery for a 3D reconstruction of a target realworld object, for example, stratification of 3D geometry, stereo and non-stereo point based techniques, surface rendering techniques, monocular cues methods, and last but not least, binocular stereo vision, some of these are mentioned in [80]. Research on these techniques are not explored in this thesis. Due to the availability of CT scanning machines, we focus on developing a generic scanning methodology that utilizes the output of DICOM data, however, the main idea of the scanning methodology should not be limited on to CT scanners.

\subsection{Game Engines and Physics Engines}

Game engines are a form of development platform used to create virtual environments for interactive video games. "Serious games" are interactive simulations made to relate or depict important information that may represent real-world events and solve problems not primarily for the purposes of entertainment. There exist serious games for health, advertisements, education, science and research for areas in defense, healthcare, marketing and communications, education 
and businesses. Serious games that have been created include health related simulations for nursing [81-83], dental [84], surgery [85], disaster planning/mitigation [84], and urban planning [86].

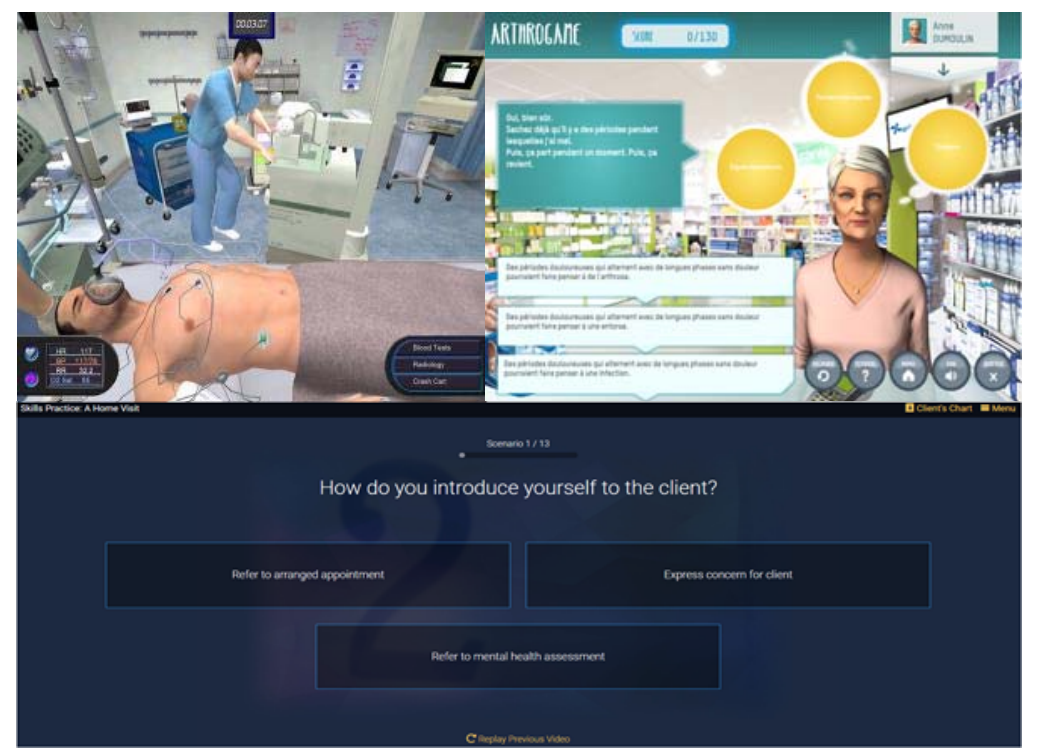

Figure 2.12 - Examples of serious games developed for healthcare: serious game for surgery (top-left), serious game for pharmacy (top right), and serious game for nursing (bottom) [12].

A game engine allows a developer to create simulations and add functionality using tools not available in alternative visualization schemes. The realism that can be depicted, using simulated physics, improvised graphics rendering, and/or real-world videos, have made these platforms plausible research tools especially when an environment is particularly complex and the ability to recreate it is impractical by other means.

A game engine allows the re-use of components, high-end graphics rendering, and most importantly, accurate simulated physics. The backend components provided to a programmer can differ between engines, however, common features typically include; physics (gravity, virtual mass, virtual dimensions/pose, etc...), rendering (mesh rendering, projectile rendering, etc...), 
audio (surround sound, special effects sounds, etc...), graphical user interface (overlay of various screens), and networking (protocols for multiplayer interaction over a network).

This functionality is conveniently accessed through high-level application programming interface (API) calls (a set of routines, protocols, and tools for building software applications). Collections of prefabricated 3D models and prewritten code can be added to any "scene" (game environment) or "player" (game actor). This degree of modularity allows for re-use of resources and a reduction in development time.

\subsubsection{Unity Game Engine - Game/Simulation Development Platform}

Unity 2017.3 is a proprietary game engine developed by Unity Technologies, primarily used to develop cross-platform video games and simulations for computers, consoles and mobile devices [87]. This engine is developed in $\mathrm{C}$ and $\mathrm{C}++$ and offers an integrated development environment in $\mathrm{C} \#$ and JavaScript. The engine is primarily designed for operating systems such as, Windows, Linux, Mac, iOS and Android, but is compatible with other industry-leading platforms such as PS4, Xbox, and WiiU.

Unity features two different Physics Engines: NVidia PhysX for 3D physics and the Open Source project Box2D for 2D physics. It is compatible with Direct3D (Windows), OpenGL (Mac, Windows, Linux), and OpenGL ES (Android, iOS). The game engine's native graphics APIs supports Vulkan, iOS Metal, DirectX12, NVidia VRWorks or AMD LiquidVR. A wide variety of 3D model file types are supported allowing the use of a range of modeling packages such as 
Blender [88] and Meshlab [89]. Unity is capable of simulating real-world physics to a high degree of fidelity, making it suitable for its use as a serious game platform.

The Unity engine can be configured to render relatively low and computationally lessexpensive computer graphics (resulting in a low cost of visual effects), but with a unique particle system that can be customized and modified in a manner that suits the requirements of a highlyaccurate representation of real-world physics and interactions of different objects.

Many serious learning simulations have been developed using Unity, and it is one of many game engines that can implement additional customized physics emulation processes for simulating physics.

\subsubsection{Physics Engines}

The component that provides simulated but realistic interactions within a game engine environment is the physics engine that runs in the backend of the game engine. The physics engine handles the mathematical functions related to physical interactions. Typically a physics simulation is broken down into two areas: collision detection and dynamics, with the assumption that time is iterating in fixed values of time,; which value is set to 20 milliseconds by default ( 50 updates per second) [90].

Collision detection determines which objects contact each other through their behavior. Dynamics is concerned with the dynamic motion of objects that exist independently or are affected by constraints, like joints (two objects connected at some point). The detailed processes of these 
calculations are abstracted to high-level API calls. In a game engine, these function calls are nontransparent and are of no concern to a developer when creating an application. The developer simply provides object parameters (i.e. mass, geometric dimensions, etc.) to be used within the engine, this whole process however, can be overridden in Unity by customized scripts. The accuracy of the physics layer within simulators is essential in providing realistic models of the outside world.

A comprehensive review of physics engines for simulations can be found in [91], which compares engines such as Open Dynamics Engine (ODE), Newton and NVidia PhysX in a controlled simulation setting to determine accuracy in a variety of physics-related themes such as collision detection, hard (rigid) and soft body dynamics, particles, and load-bearing. The current industry standard for physics are engines such as NVidia PhysX, Havok and most recently, NVidia FleX [92].

NVidia FleX is a position-based particle simulation designed to mimic real-world interactions, with the use of specialized algorithms, such as ellipsoid splatting. These algorithms are designed primarily for visual effects such as moving rigid bodies, fluids, clothing etc. However, they are not necessarily a physically accurate simulation of the real-world.

For the purposes of IED neutralization serious gaming, a game engine is required that has a customizable particle system capable of accurately representing real-world physics and physical interactions of matter. 


\subsubsection{Serious Game Simulation}

A serious game is generally designed to closely simulate aspects of the real-world [84]. It can run on a single computer or a network of computers and it uses abstract computational models to simulate behaviors of a real or fictitious environment. By having the ability to simulate a particular environment with accurate computational models of real-world settings, it can be used to gain additional awareness of a system [93].

An example simulation is a forensic analysis simulation to estimate projectile trajectory and projectile's penetration strength - computational models can be applied to the simulation to calculates a projectile's impact on another object with added parameters such as weight, density, velocity and trajectory. By applying computational physics, computer-based experiments using valid computational modelling of systems are used to perform inference of a certain type of behavior.

A serious game simulation can be an education-purposed methodology requiring a player to solve a problem and being evaluated while doing so [12]. A selection of simulations for training and educational purposes can be found in my previous master's work [21]. These simulations imitate real world processes to see how they behave under different conditions and can also be used to test new theories. As of this writing, there has been no simulation created to model neutralization dispersion, but we model the dispersion process using fundamentals of high velocity projectile impact simulations which will be discussed in detail in section 2.6 . 
A serious game simulation is one that correctly demonstrates an ability to replicate actual real-world patterns and behaviors and can provide reasoning which can conceivably be applied to real-world applications. In order to create a credible and accurate simulation, models in the simulation must be calibrated (achieved by adjusting any available parameters in order to adjust how the model operates and simulates the process), verified (achieved by obtaining output data from the model and comparing them to what is expected from the input data) and validated (achieved by comparing the results with what is expected based on historical data from the study area).

To measure the performance of a serious game, the common research challenges in designing an effective serious game lie in the reception of the game (student appeal and motivation) and focus of the developmental skill (for example language-based learning or technical skill based learning). The best practices for effective game based-learning follow a number of key principles such as rewards, feedback, challenges, goals, rules, choice, fantasy, fidelity, context and constructivism. A detailed explanation of each principle is provided in [21].

The next section provides a brief review of high velocity impact simulations as it pertains to one of the contributions of this thesis - analyzing impact points as the result of a successful shot neutralizing a target.

\subsection{High Velocity Projectile Impact Simulation}

The disruption process, being highly energetic and somewhat unpredictable, has the potential to impact surrounding infrastructure and may cause harm to people from the dispersed 
debris and scattered shrapnel which can travel up to a 20 meter radius [94] depending on the power of the propellant charge used and direction of the disrupter cannon.

It is important for EDU to plan for the dispersal of the components from the effects of the disrupter shot to identify possible impact areas where damage and harm to property and people may occur. Therefore, research in advancing safe and beneficial approaches to neutralization operations benefits both members of the EDU community and the general public.

A serious game simulation for IED neutralization will provide EDU with additional information on the potential physical dispersion of the components of an IED and any major points of impact (splatter) as a result of a high velocity water projectile being used.

\subsubsection{Hydraulic Shock/Ram}

HRAM is a phenomenon that occurs when a highly-energetic object penetrates a fluid filled container [13]. This area of research analyzes the vulnerabilities of aircraft or vehicle fuel tanks [95] to projectile impacts with objectives that range from determining failure stages of a water-

filled tank of a specific construction (thickness and size) and material [96], to analyzing the energies, momenta and pressure contours from an applied resultant force [97].

Many of these studies have numerical models, analytical models, finite element models, simulations and mock experiments, and real-life experiments contributing to findings, which are applied to a number of different objects such as aluminum tubes, thin-walled, liquid filled containers and containers with varying amounts of fluid and granular fillings [98].

\subsubsection{Particle Momentum Explosive Effects}


Zhang et al. [99] studied a spherical charge that generated a blast wave of complex supersonic gas-solid flow, and used a flow topology algorithm to model the dispersal process from a dense granular flow to a dilute gas-solid flow. The models and experiments of [98], [100], provide numerical and analytical results that utilize gas and aqueous governing equations to solve the dissipation of particles from a shock wave.

The current research in high-velocity projectile impact model the dispersion of particles in a closed system environment with at most one target entity (e.g. a fuel tank containing liquid or a half-filled aluminum tube).

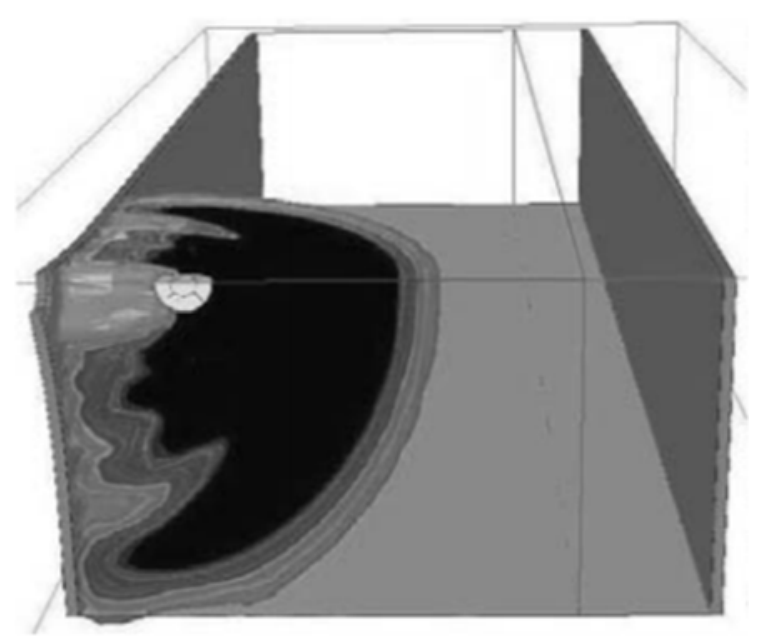

Figure 2.13 - Pressure map simulation of a projectile at a velocity of $900 \mathrm{~m} / \mathrm{s}$ [13] in a simulated closed fuel tank.

\subsection{Continuum Mechanics}

The underlying assumption of these models is continuum mechanics [101], which analyzes the kinematics and mechanical behavior of materials modeled as a continuous mass rather than as discrete particles. 
In continuum mechanics, it is sufficient to use computational fluid dynamics models to analyze the transfer of a projectile's momentum and kinetic energy through the fluid to the surrounding structure. For the purposes of our study, these models are insufficient to accurately model multiple objects of varying density, size and shape, as these entities are separate 'discontinuous' and disconnected masses - which are often characteristics of a suspect IED, as shown in Figure 1.4.

This limitation is shown in our previous work [23], which is a dispersion of shrapnel utilizing the theory of continuum mechanics and models of the dispersion's primary effects as a closed system with only initial forces, and known densities as input parameters.

\subsection{Molecular Dynamics}

Instead of using continuum mechanics, the method that best fits our use case of modelling discrete particles, which also takes into account frequent multi-collisions is Molecular Dynamics (MD) [45]. MD is a soft-particle method that permits small overlaps between particles to simulate the elastic deformation of material elements. This phenomenon is characteristic of a high-velocity $(>700 \mathrm{~m} / \mathrm{s})$ disrupter shot. 
An improved method to simulate dispersal of particles that accurately mimics real-world physics is to consider every object in a game engine as a conglomeration of discrete solid macroscopic particles [14].

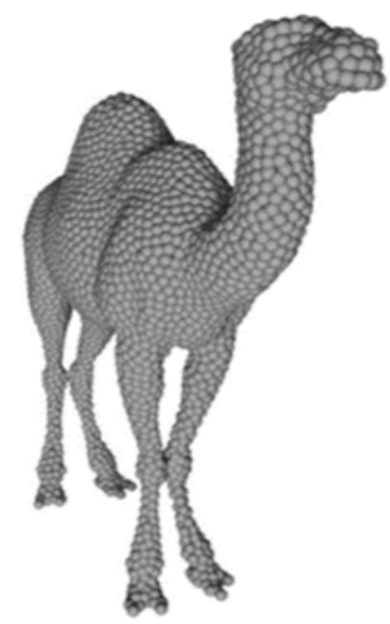

Figure 2.14 -A computationally efficient rendering of a model represented by 4147 non-macroscopic particles (top right) [14].

Particles can represent discrete elements of the simulated material, making it possible to compute each particle interaction per frame update. Each fixed update interval recomputes every physics step in the simulation. This particle-based method allows for physically accurate simulations as opposed to computed topological interaction of particles as an approximation (which means it only appears to mimic real-world effects).

The drawback of this method is a computationally expensive simulation which causes each particle to be recomputed and redrawn at every frame, or rather every second for Unity. This method also results in a significant loss in performance and uses relatively more memory.

The next chapter discusses the mock IEDs containing inert explosives, the various materials for density and mass measurements and the mock IEDs that were created and used and 
techniques used to collect our data. We also discuss the methodology used to create 3D models from DICOM data along with Skannect data is presented, along with the methodology to determine relative densities of occluded objects. Lastly, we present a particle dispersion methodology unique to the neutralization task of disrupting IED components of varying densities and masses. 


\section{Chapter 3 Technical Approach}

\subsection{Introduction}

In order to understand our algorithm (from the construction of our inert IED, to the data acquisition phase, to the development phase and the serious game phase), it is important to understand that there are numerous steps involved in the neutralization task according to EDU field experts [102], [103], and these steps may vary depending on the type of explosive, the current situation, size and shape of the suspect package, and placement of such package. One common step in these processes is to determine (if possible) the location within the package of critical components such as a power source, triggering mechanism or other non-explosive (but functionally critical) element of the device.

In addition to the pose information on these critical components, other types of information such as its relative mass, density and dimensions also make it possible to facilitate a means to neutralize the device by taking aim at the non-explosive component with a disrupter cannon. If the first neutralization attempt is determined to be unsuccessful, the process will be repeated until the threat is determined neutralized by EDU experts.

Our technical approach is best described as an algorithm that is divided into parts - with each part illustrating a contribution of this thesis.

Refer to Chapter 1 for detailed description of our contributions, with that in mind, please note that contributions 1,2 and 4, 5 are significantly augmented contributions from our original published work [29], and contributions 3 and 6 are new contributions from current work [22], [23]. 
The old contribution of our scoring feature remains relatively unchanged from our previous work, and has only brief mention in this chapter.

We have augmented/enhanced contributions 3 and 6 with a new algorithm (as published in our current work in [22], [23]). We consider this algorithm as a second part to the existing algorithm.

This algorithm has four phases:

Phase 1: Virtual IED Information Acquisition - involves the creation of mock IEDs, calibrating sensors, acquiring and analyzing data. (Contribution \#1,\#3)

Phase 2: Model and Mesh Configuration - involves the creation, cleanup and segmentation of virtual models and meshes, and associating bounding boxes. (Contribution $\# 2, \# 4)$

Phase 3: Physics Engine Model Configuration - involves the implementation of molecular dynamics, real-world physical attributes, projectile, dispersion to models, and implementation of the game actor to its associated real-world traits. (Contribution \#5, \#6)

Phase 4: Serious Game Testing and Validation - involves testing, debugging and validating the game. (Validation of our contributions)

Figure 3.1 shows an overview of our algorithm. The system begins with gathering input, which is cross-sectional DICOM data of real-world inert IEDs and inert explosives collected from a CT machine. The cross sectional data are merged to create a 3D model, which is then modified and segmented to separate key IED components of interest. T assemblage of these components are 
imported into a game engine, where bounding boxes, scoring criteria, real-world physical attributes are associated with each component, and each component is programmed with dispersion model mechanics that adhere to molecular dynamics. Last, our serious game is tested and validated and the next chapter will discuss the comparison of our results to real-world neutralization experiments. 


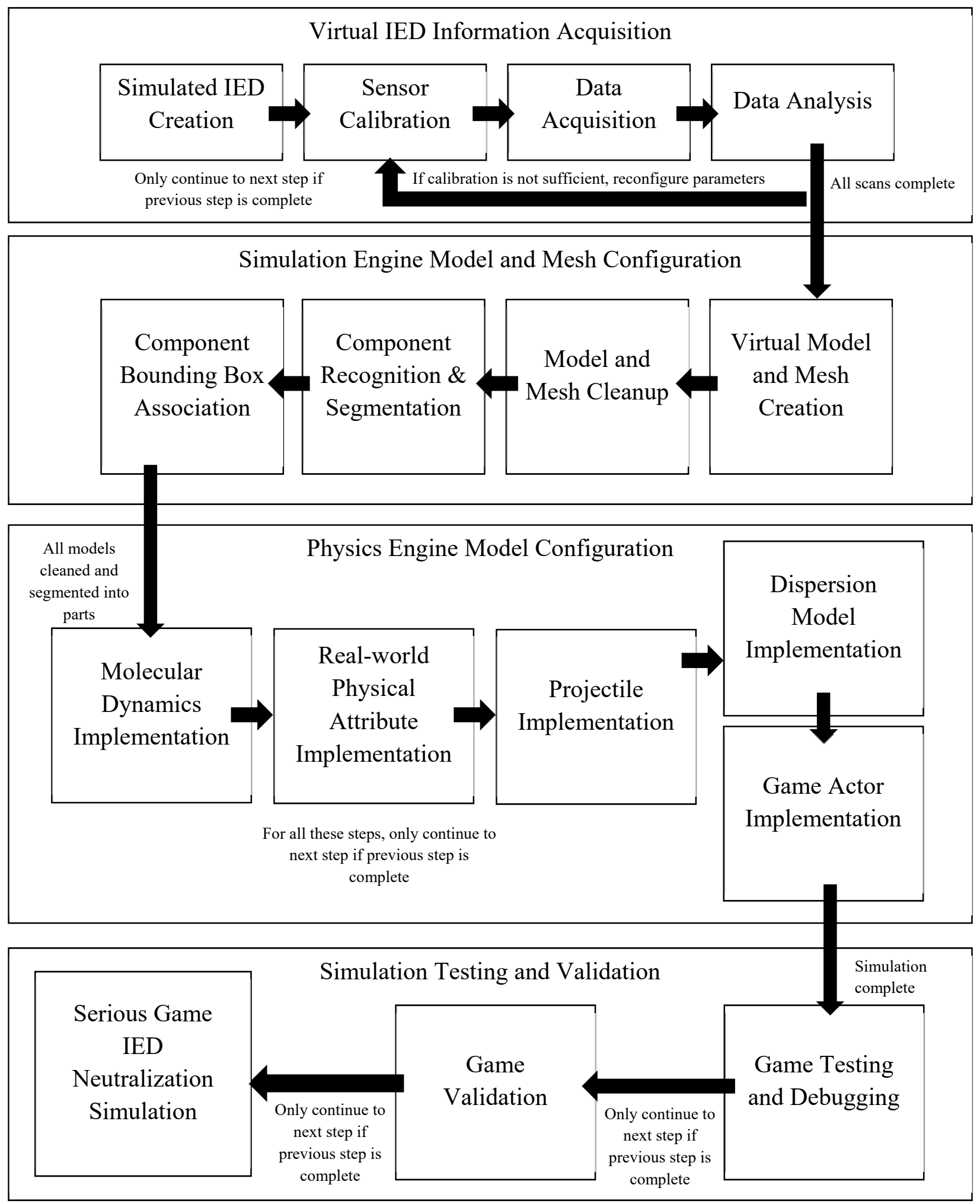

Figure 3.1 - A flowchart depicting the creation of virtual IEDs from real-world suspect IEDs to the implementation of accurate physics-based models leading to a serious game simulation. 
The next few sections describe each phase as they form the basis for the simulations.

\subsection{Virtual IED Information Acquisition}

In this phase, we describe the materials used for the creation of our virtual IEDs, as well as other sources of information (for sensor calibration purposes) gathered from common household substances and inert explosives. We also cover how the data was acquired, and how we calibrated the CT sensor to capture the range of materials that is relevant in providing our simulation with information relating to the possible type of explosive that was scanned. If we analyzed the data and we decided that the sensors needed to be recalibrated (may be due to poor visual image quality or the sensor was emitting too high/low of radiation energy to penetrate thick surfaces), then we would recalibrate the sensor and redo the data acquisition phase.

\subsubsection{Virtual Improvised Explosive Device Creation}

The main source of our data is gathered from 6 inert IEDs. The other source of our data is

7 common substances (honey, corn syrup, detergent, water, vegetable oil, lamp oil, rubbing alcohol), and 6 inert explosives (smokeless black powder, nitro dynamite, TNT cast booster, M112 block (C-4) Assembly, pentaerythritol tetranitrate (PETN) and Semtex 10 Assembly) purchased from DSA Detection [104], [105] and used for sensor calibration and validation.

Figure 3.2 describes the design, purpose and arrangement of our mock IEDs, as well as the purpose of the common household substances and inert explosives used for sensor calibration. 


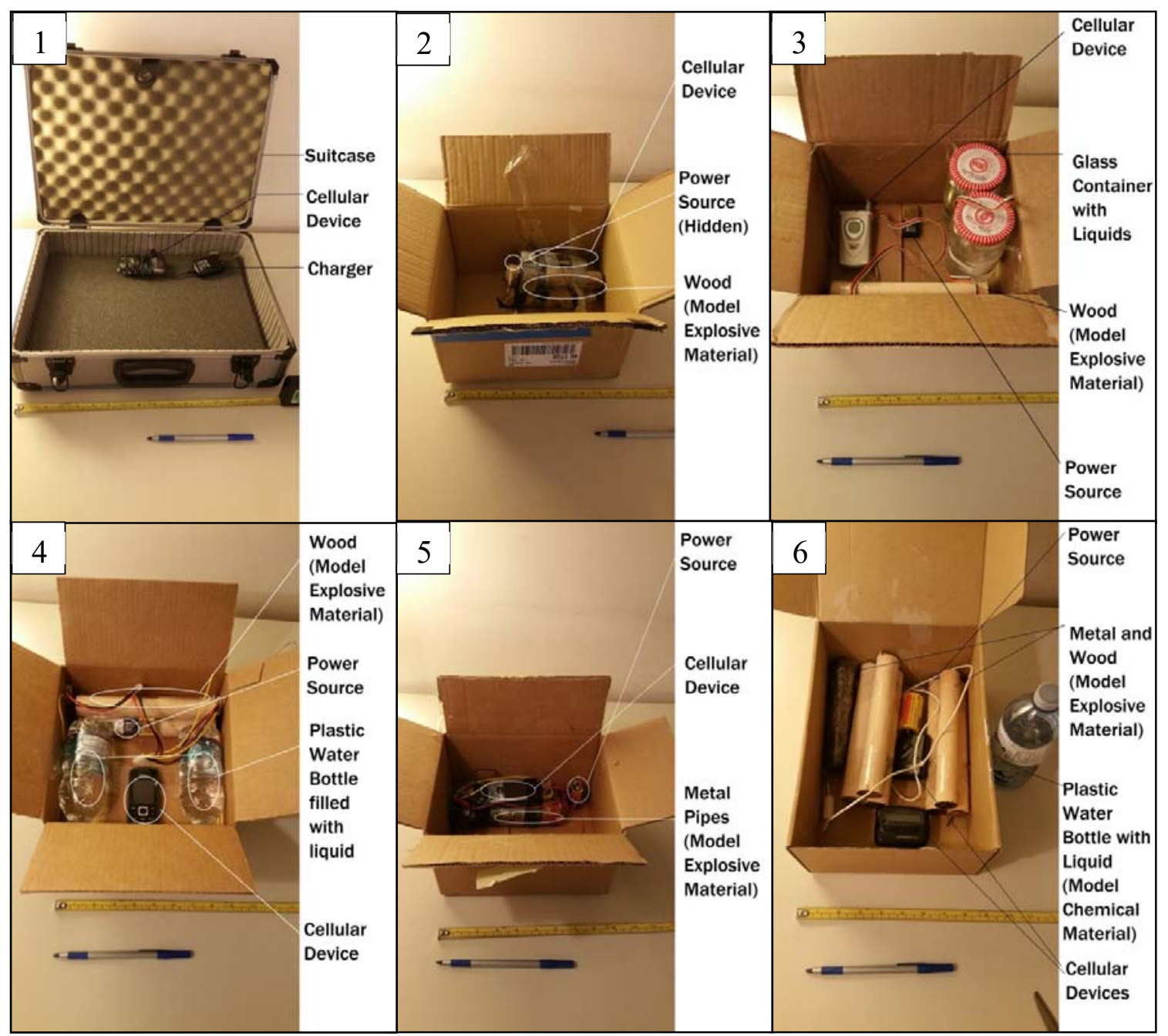

Figure 3.2 - Revealed mock IED components in cardboard containers. 1. Metal/plastic suitcase with a cell phone (triggering mechanism) and 2-6. Cardboard container with IED components with varying types of material (for example metal, plastic, glass, or wood) in a variety of arrangements so the IEDs are random and improvised.

The mock IEDs, shown in Figure 3.2, are constructed for the purposes of this thesis, and follow not-atypical IEDs designs (providing a significant degree of variety), consisting of various types of materials (broad coverage of different materials such as metal, plastic, glass, and wood) and in various arrangements and compositions.

All IEDs are constructed consisting of one of each critical component that make up a basic IED. These 4 components common in most IED designs, according to EDU experts [102], follow the acronym PIES: Power, Initiator, Explosive and Switch. In each design, we placed a battery to 
represent a power source, wires and fake blasting caps to represent the initiator, cylindrical wooden blocks, or substances in water bottles to represent explosives and a cell phone/pager/timer to represent a switch. The assembly of these components are wrapped in electrical tape - these components were chosen from commonly available material with varying densities which are characteristic of IEDs according to EDU specialists [102]. These IEDs were placed within 12 x 10 x 15 inch banker boxes.

The 7 different common substances with their associated densities are defined in Table 3.1.

\begin{tabular}{|c|c|}
\hline Substance & Density $\mathbf{( g / \mathbf { c m } ^ { \mathbf { 3 } } )}$ \\
\hline Honey & 1.42 \\
\hline Corn Syrup & 1.33 \\
\hline Detergent & 1.06 \\
\hline Water & 1.00 \\
\hline Vegetable Oil & 0.92 \\
\hline Lamp Oil & 0.81 \\
\hline Rubbing Alcohol & 0.79 \\
\hline
\end{tabular}

Table 3.1 - The common substances and their densities used in calibrating the CT sensor.

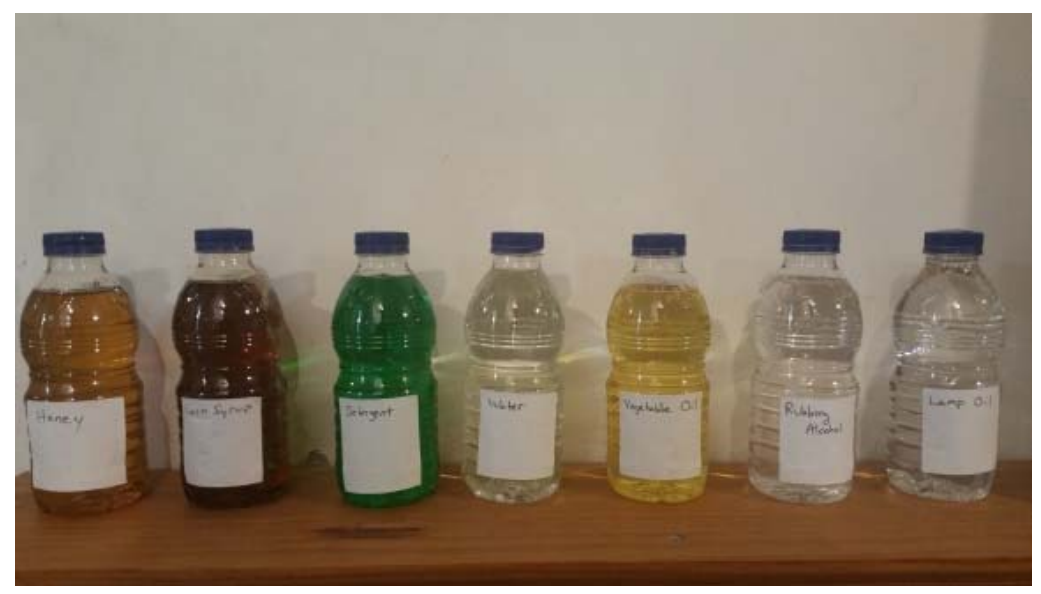

Figure 3.3 - From left to right, the liquids shown are honey, corn syrup, detergent, water, vegetable oil, lamp oil and rubbing alcohol, each chosen for their distinct densities.

We choose to use these 7 substances because they are typical household common substances that are readily available and may be purchased from most stores - which means sensor 
calibration and experiments may be relatively easy to reproduce. The density of each substance is verified and known prior to testing.

The substances are stored in $330 \mathrm{ml}$ clear water bottles and the bottles are chosen because they have been verified to not show any discernable visual traits in an output of a CT scan. The purpose of these bottles is to house the substance, and provide negligible visual surfaces shown in images of CT scans. The purpose of using these substances in scans are to provide a frame of reference when we begin data acquisition - the relative density measurements of these common substances will be compared and associated with the relative density measurements of inert explosives to produce a model to classify relative unknown densities of unknown substances.

It is important to have a baseline substance so we can reference other substances and provide relative density measurements when performing the sensor calibration process - we use water which has a density value of $1.00 \mathrm{~g} / \mathrm{cm}^{3}$ as our baseline substance.

Other substances with known densities may be substituted or added to this sensor calibration process, but any substances must not be combined with another substance because of the added complexity (each substance must be placed in individual containers), and should have a distinct known density measurement that should provide a range of density values (with differences of $>0.10 \mathrm{~g} / \mathrm{cm}^{3}$ or $<0.10 \mathrm{~g} / \mathrm{cm}^{3}$ ).

The 6 inert explosives are shown in Figure 3.4. These are inert explosives purchased from a United States security company named DSA Detection [105] that manufactures and sells EDU aids. 
We have chosen to use high-grade inert explosives that are designed as physical replicas of real explosives. These inert explosives are designed to closely match the critical features, density, effective atomic number (Zeff), colour and texture of the real explosive. These inert explosives are verified to respond on most airport security screening systems and trigger alerts on automatic detection systems that use X-ray and CT sensing technology.

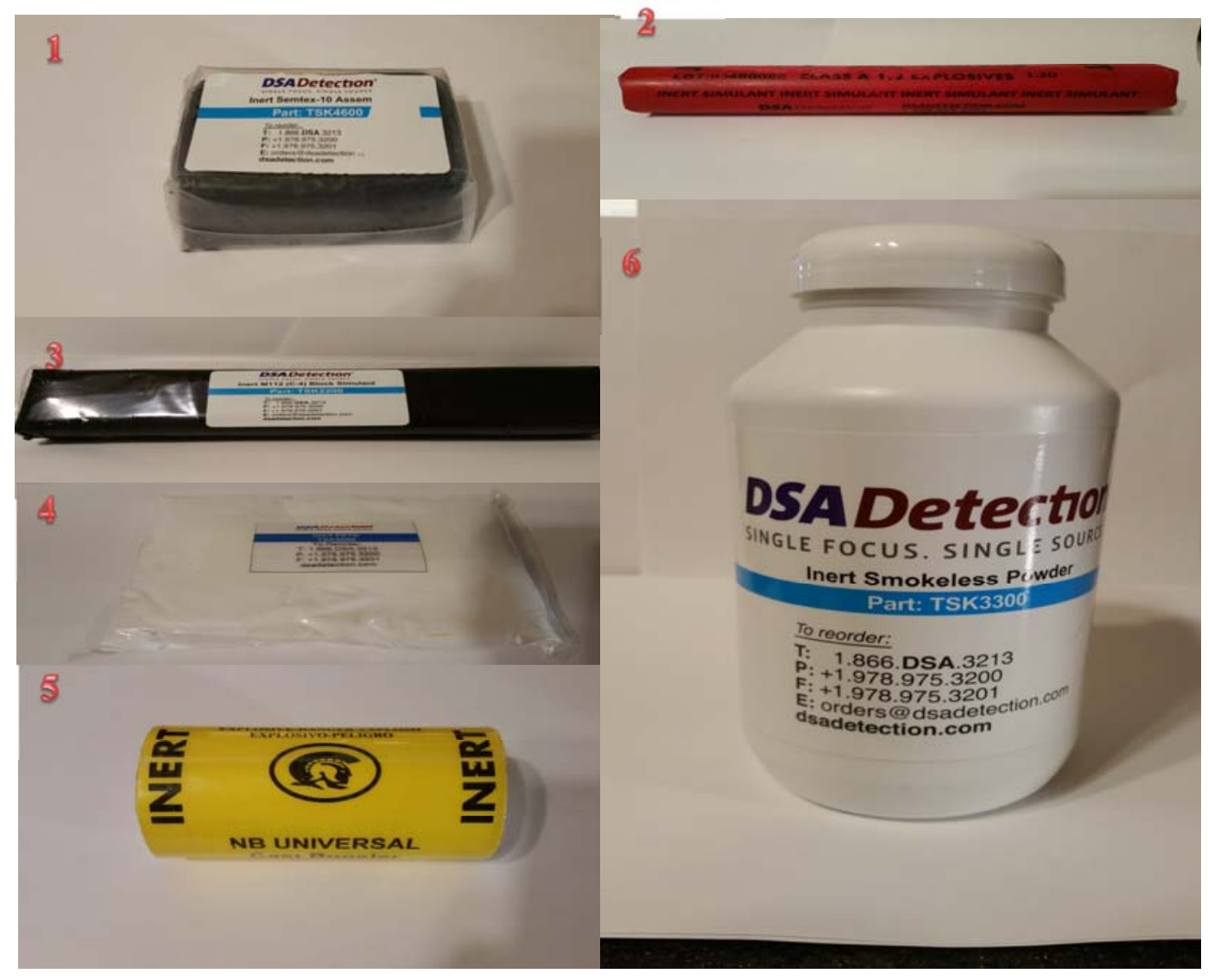

Figure 3.4-\#1. Semtex 10 Assembly. \#2. Nitro dynamite. \#3. M112 block (C-4) Assembly. \#4. PETN. \#5. TNT cast booster. \#6. Smokeless black powder.

\subsubsection{Sensor Calibration}

As mentioned in section 2.3.2, regarding CT scanners, results obtained from scanner to scanner vary significantly, even when the same brand and model of scanner is used. So in order to 
obtain relevant information, we must calibrate the $\mathrm{CT}$ sensor to obtain specific values that are associated with specific parameters and a particular scanner. Following this procedure will enable a measurement of relative densities of target objects, and these relative values will be useful to our process as long as the same scanner and parameters are used to scan subsequent targets.

We begin the sensor calibration process by adjusting the $\mathrm{CT}$ scanner according to the parameters in the head and neck protocol [106]. In our tests, we employed the Philips Big Bore CT [107] with Pinnacle3 [15] as the treatment planning system. The use of alternate CT scanners are also possible but should follow the AAPM CT lexicon translation chart to discern important CT terminology and parameters of different manufacturers' systems [108].

The technical parameters [109] that are often adjusted are:

- Tube current-time product (mAs) - The product of tube current and exposure time per rotation, expressed in units of milliampere * seconds (mAs).

- $\quad$ Slice thickness (in millimeters) - Nominal width of reconstructed image along the $\mathrm{z}$ axis.

- Resolution - The ability of the imaging modality to differentiate two objects. Low spatial resolution standards will be unable to differentiate between two objects that are relatively close together.

- Field of measurement (or calibrated field of view FOV) - The diameter of the circular region within the scan plane over which projection data are collected. 
In addition, these parameters must be calibrated according to the size and density of the target in order to produce reasonable output, an expert scanning technician assisted in configuring the parameters of the scanner. Failure to calibrate according to the target would cause the output images to suffer from excessive noise which can hamper the detection process.

The parameters we used are shown in Table 3.2, other parameters are normally not adjusted in the head and neck protocol.

\begin{tabular}{|l|l|}
\hline Head \& Neck & Values \\
\hline FOV & $500 \mathrm{~mm}$ \\
\hline View Angle & Multi Surview \\
\hline $\mathrm{kV}$ & 120 \\
\hline Tube current & 30 \\
\hline Thickness & $3 \mathrm{~mm}$ \\
\hline Increment & 3 \\
\hline mAs/slice & 300 \\
\hline Resolution & standard \\
\hline Filter & standard $(\mathrm{B})$ \\
\hline
\end{tabular}

Table 3.2 - Head and neck parameters taken from the standard AAPM head and neck protocol, optimized for dense objects.

The parameters in Table 3.2 are defined as follows [108]:

- Field of measurement or field of view (FOV) is the diameter of the circular region within the scan plane over which projection data are collected.

- Multi surview is the scanned projection radiograph, often acquired by the CT system to allow the user to prescribe the start and end locations of the scan range.

- Tube potential $(\mathrm{kV})$ is the electric potential applied across an $\mathrm{x}$-ray tube to accelerate electrons towards a target material, expressed in units of kilovolts $(\mathrm{kV})$. 
- Tube current is the number of electrons accelerated across an x-ray tube per unit time, expressed in units of milliampere $(\mathrm{mA})$.

- Thickness is the nominal width of reconstructed image along the $\mathrm{z}$ axis.

- Increment is the distance between two consecutive reconstructed images.

- Tube current-time product (mAs/slice) is the product of tube current and exposure time per rotation, expressed in units of milliampere seconds (mAs). In axial scan mode, this is equal to tube current $\times($ scan angle $\div 360) \times$ rotation time. In helical scan mode, this is equal to tube current $\times$ rotation time.

- Resolution is the pre-defined standard dimensions of the images before 3D reconstruction.

- Filter is the pre-defined standard image modifications to alter sharpness or smoothness (done in image space without reconstructing images)

The CT operator needs to input these parameters into the CT planning software system Pinnacle [15], which is connected to the $\mathrm{CT}$ scanner, subsequent parameter changes require recalibration of the CT scanner using the same computer and software. 


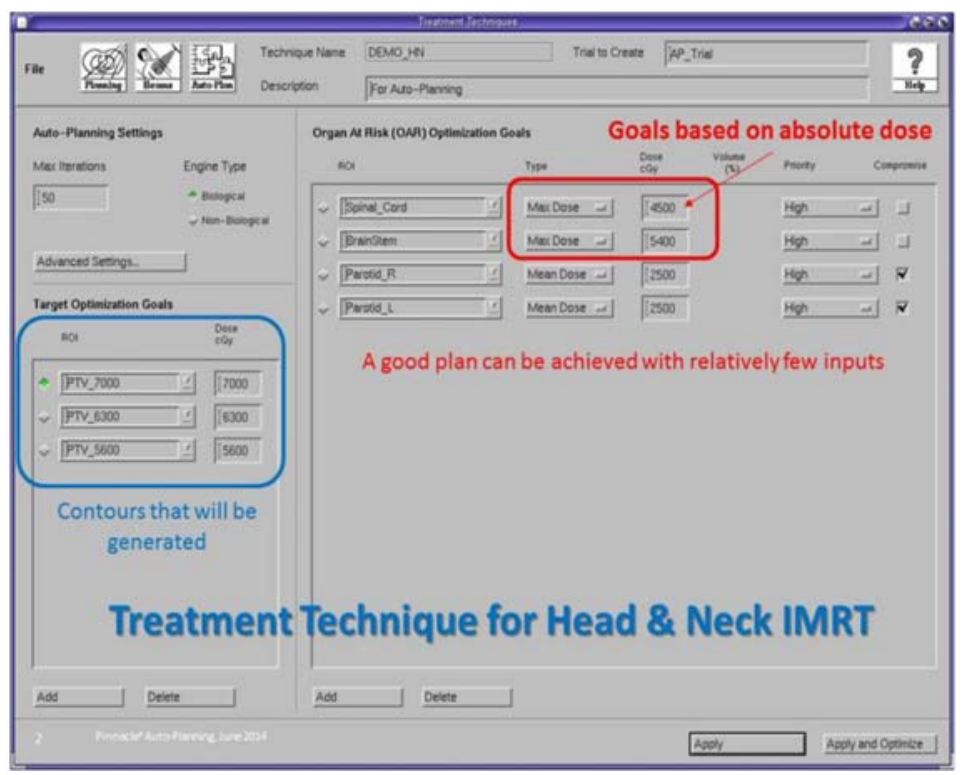

Figure 3.5 - A sample treatment planning screenshot of Pinnacle by Philips [15], often cluttered with tedious and repetitive steps and parameters, requiring significant planner/physician interaction. Plan quality varies depending on the experience of the operator.

\subsubsection{Data Acquisition}

After calibrating the sensor to match the parameters in the head and neck protocol, we place the common substances together in a $50 \mathrm{~cm} \times 50 \mathrm{~cm}$ wide cardboard container and place it into the CT scanner. We ensure that the same cardboard container is used for subsequent scans.

The head and neck protocol is meant to scan minute details $(<1 \mathrm{~mm}$ allowable degree of error) within the cranium [106]. To closely mimic this situation, and also to scan our materials at this level of detail, we use cardboard, which we deemed appropriate for its similar surface and thickness to that of a cranium - so that radiation rays from the sensor's emitter can penetrate the surface of the cardboard container, and reach the interior objects at the relatively same radiation energy as it penetrates through cranium. Without the absorption of the radiation from the cardboard container, the results will differ, (the scanned image may appear too saturated) and the sensor will need to be recalibrated to obtain a more notable range of photometric brightness. 


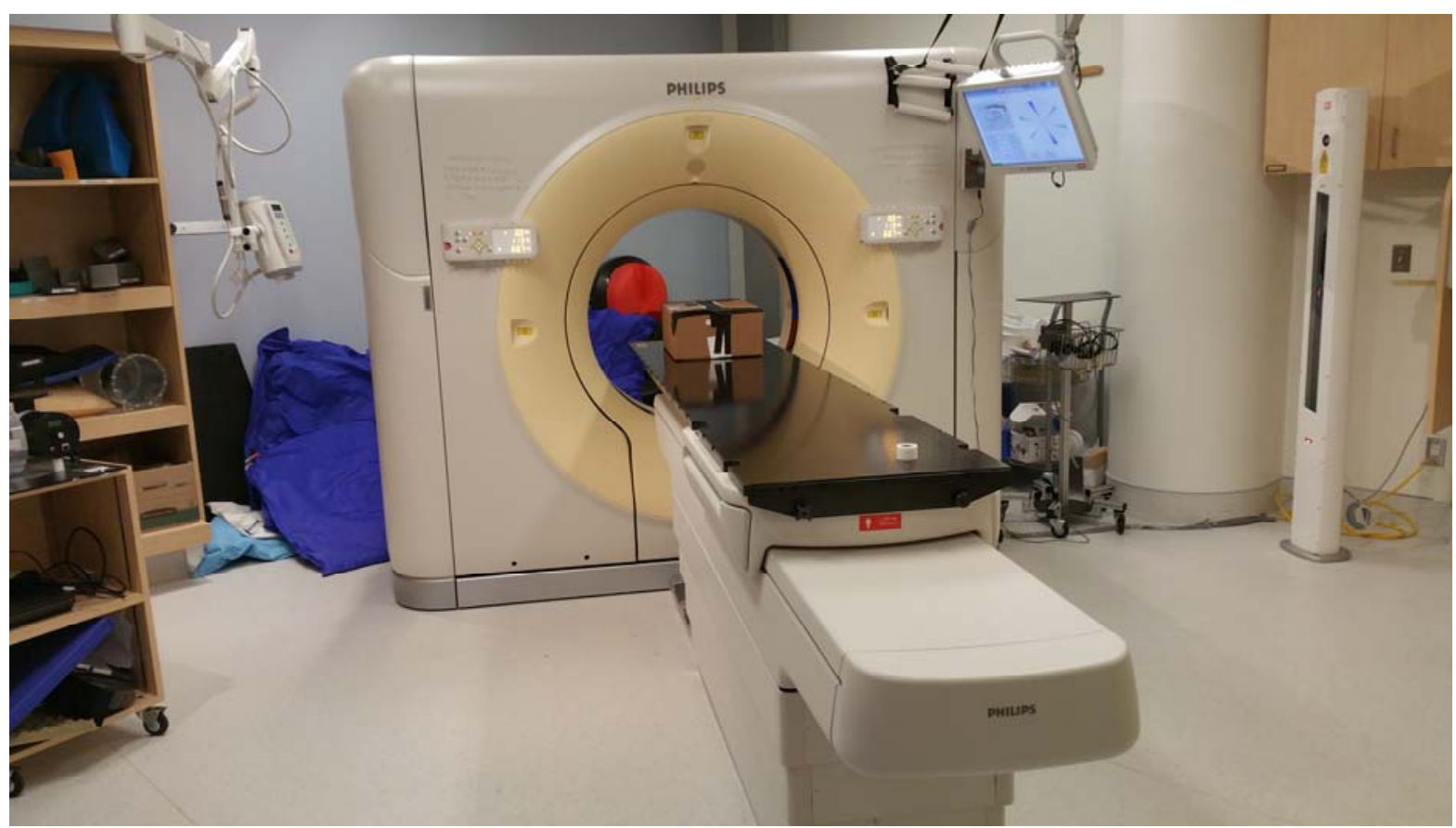

Figure 3.6 - An image of the Philips Big Bore CT sensor with the target package of substances being scanned.

Any reasonable container may be used to house the common substances and inert explosives, as long as the same container is used in subsequent scans with the same protocol and scanner.

Our research used the clinical CT scanning machine named Brilliance Big Bore developed by Philips, we captured data on 3 planes, with a field of view of 500mm, slice thickness and spacing of $3 \mathrm{~mm}$ and $2 \mathrm{~mm}$. These are certain parameters that are most important when using the CT scanner according to a CT technologist [79]. 
For simplicity sake, these technical parameters (tube current-time product (mAs), slice thickness (in millimeters), resolution, and field of measurement (or calibrated field of view FOV) can provide the operator a significant leverage in the amount the radiation exposure and level of image quality.

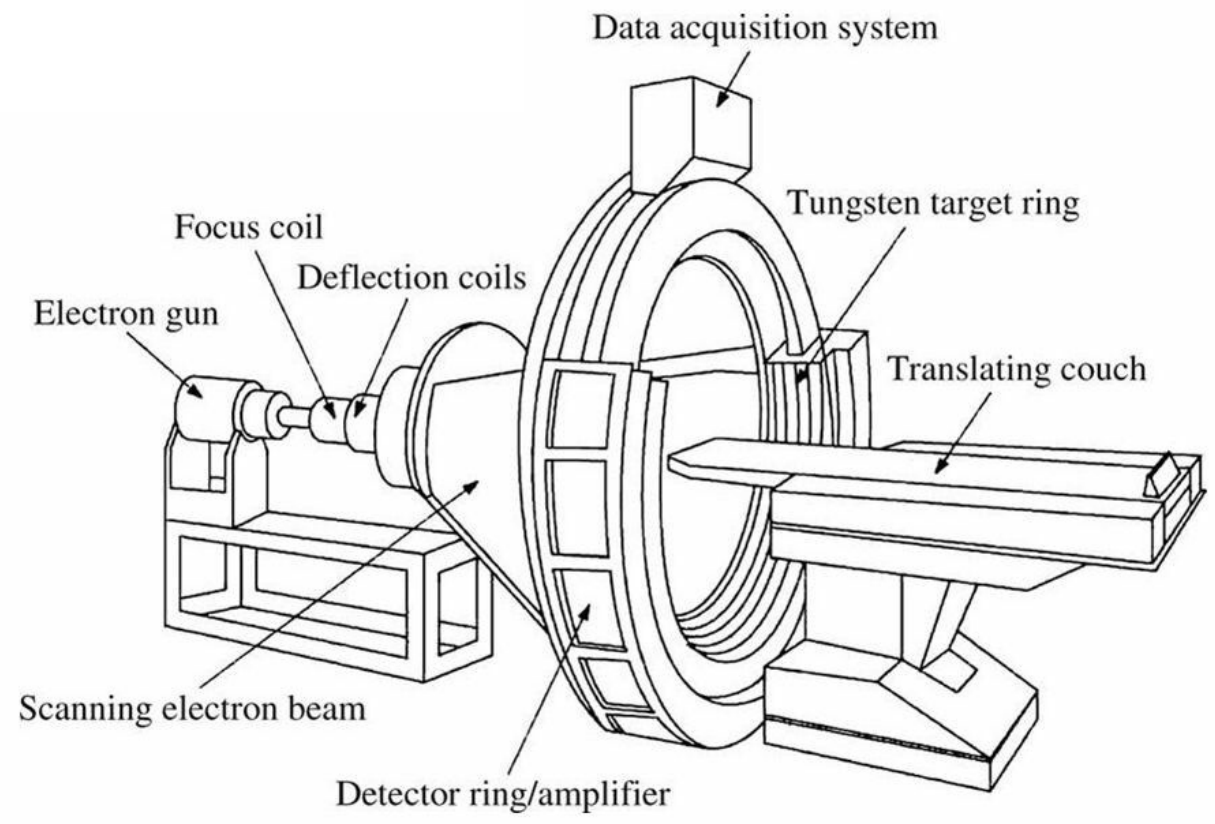

Figure 3.7 - A diagram of a CT scanning machine [16].

The images were captured at resolutions of 512x512 pixels and range from 300-400 slices depending on the number of objects placed on the patient table. As described in a medical journal [110], adjusting these parameters affect 3 primary factors when performing a scan; signal-to-noise ratio, resolution and scan time. The signal-to-noise ratio measures the quality of the image, essentially, the lower the noise, the better the result. When using the CT scanner, the less time spent scanning, the lower the resolution and the higher the SNR. 
According to CT technologists [79], [111], with these parameters, the resulting visual representation of the objects from the DICOM data can effectively capture the soft materials (cardboard, liquid and plastic) and hard materials (metal and wood). See Figure 3.8, Figure 3.9 and Figure 3.10 for a 3D representation of the mock IEDs from the DICOM data.

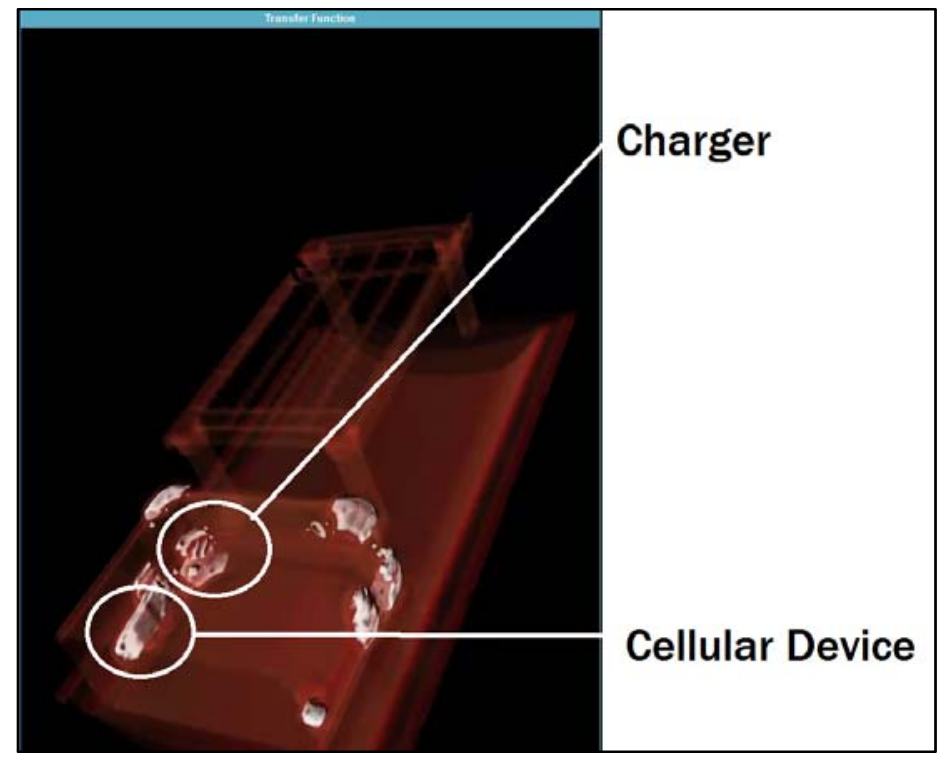

Figure 3.8-3D representation of the suitcase and support platform from DICOM data.

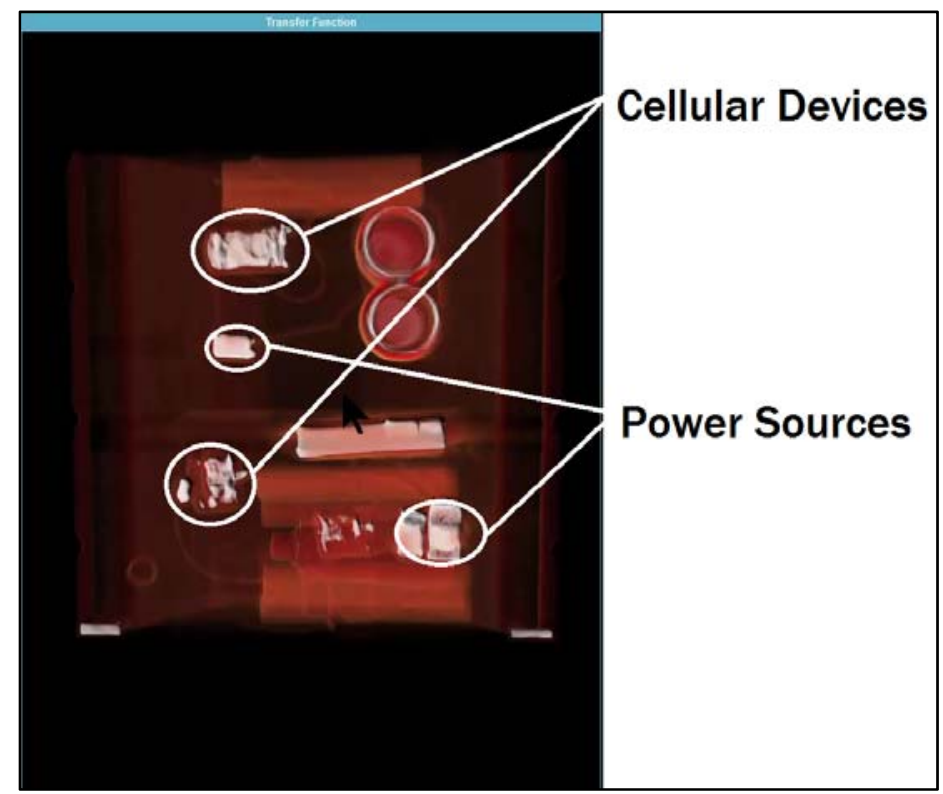

Figure 3.9-3D representation of box 3 and 6 from DICOM data. 


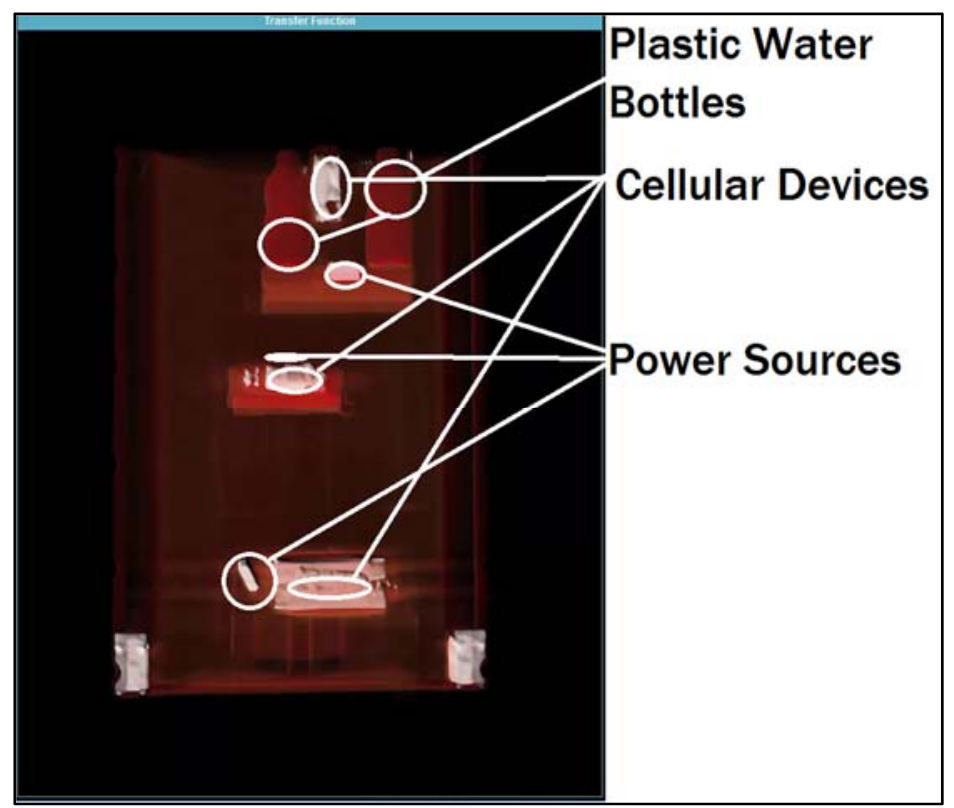

Figure $3.10-3 \mathrm{D}$ representation of box 2, 4 and 5 from DICOM data.

The inert IEDs are also scanned using the same method, but only after the sensor is calibrated and verified in the analysis stage - when the sensor is properly calibrated, theoretically, the photometric brightness values obtained from the CT scan image can be confidently used as a relative measurement of density.

\subsubsection{Data Analysis}

Each CT scan provides a range of photometric brightness values to denote relatively high or low density objects in the image. We obtain and associate photometric brightness values, with each substance's known density. A sample 2D cross sectional output of a CT scan output is shown in Figure 3.11. 

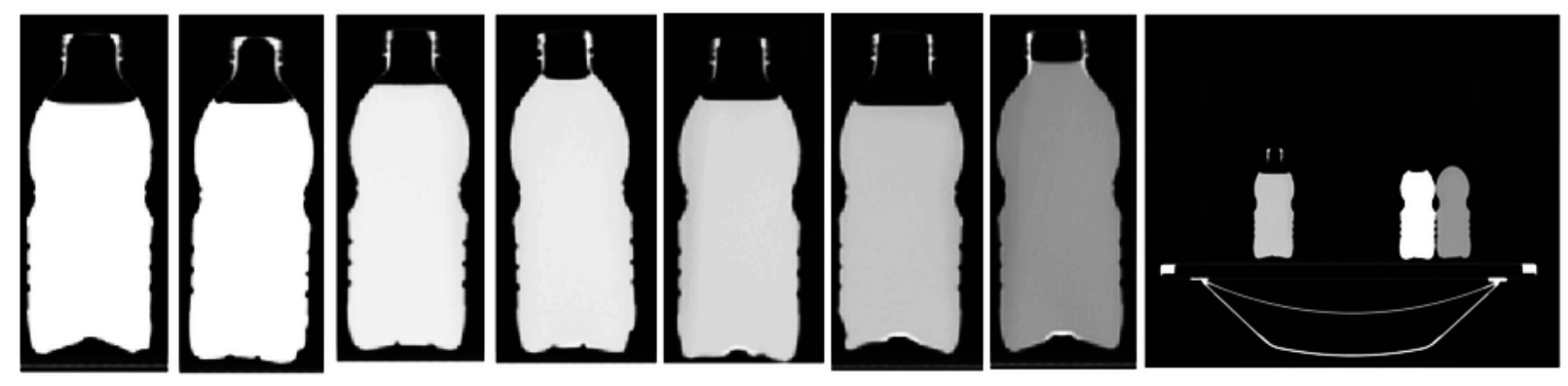

Figure 3.11 - A 2D cross sectional output of a CT scan of the following household common substances: left to right: the liquids shown are honey, corn syrup, detergent, water, vegetable oil, lamp oil and rubbing alcohol, each showing up on the scan as different light intensities as a result of their different densities.

In order to gauge photometric brightness, we utilize the hue, saturation and value (HSV) colour space - most commonly used in computer vision techniques to separate 'luma', or the image intensity, from the color information.

Since we are only concerned with brightness dimension, we extract the luma component $\mathrm{V}$, which is given as a real number between 0 to 1 - or in other words, it seems to appear as black at 0 and white at 1 . In this thesis we refer to the value component as brightness.

For each region of each substance, we calculate the average brightness and associate it with the density of the substance.

The data values for Figure 3.11 are shown in Table 3.3, we arrange these data values from least to greatest and design a lookup table to categorize other data values. The brightness value for water acts as our baseline substance. As a heuristic, the CT scanning parameters are calibrated such that the brightness value is near 0.5 - a median brightness value in the HSV color space. 
This heuristic could ensure that the variety of substances more dense or less dense than water can be represented in the HSV color space. If the brightness value for water is near 1, then the parameters should be adjusted accordingly to obtain a lower baseline value for water.

\begin{tabular}{|c|c|c|}
\hline Substance & Density $\mathbf{( g / \mathbf { c m } ^ { 3 } )}$ & Brightness Value (in HSV color space) \\
\hline Honey & 1.42 & 0.9998 \\
\hline Corn Syrup & 1.33 & 0.9993 \\
\hline Detergent & 1.06 & 0.8519 \\
\hline Water & 1.00 & 0.6298 \\
\hline Vegetable Oil & 0.92 & 0.4214 \\
\hline Lamp Oil & 0.81 & 0.3239 \\
\hline Rubbing Alcohol & 0.79 & 0.0062 \\
\hline
\end{tabular}

Table 3.3 - The value obtained from the average of HSV pixel segment of the substance.

We then map density values to brightness values. Depending on the lookup table, although limited, we gauge whether our calibration of the CT scanner is optimized for reasoning about densities of unknown masses. If the lookup table has generally consistent the data points that are equal distances from each other, then the CT scanning parameters are acceptable and calibration is complete.

We then scan inert explosive to obtain range of relative brightness. After obtaining these values, we undertook scanning 6 high-grade inert explosives designed as physical replicas of real explosives [105]. These inert explosives closely match the critical features, density, effective atomic number (Zeff), colour and texture of the real explosive. We show the inert explosives in Figure 3.4, and we perform CT scans of the following inert explosives; smokeless black powder, nitro dynamite, TNT cast booster, M112 block (C-4) Assembly, pentaerythritol tetranitrate (PETN) and Semtex 10 Assembly—all available through DSA Detection [105], the stated densities for these inert explosives are taken directly from DSA Detection product information. 
Figure 3.12 shows the multi-planar reconstruction of scans from 3 planes; sagittal, axial and coronal views of the inert explosives. We segment parts of image slice that correspond to the inert explosive and then extract the average brightness value from the HSV colour space of that segment.

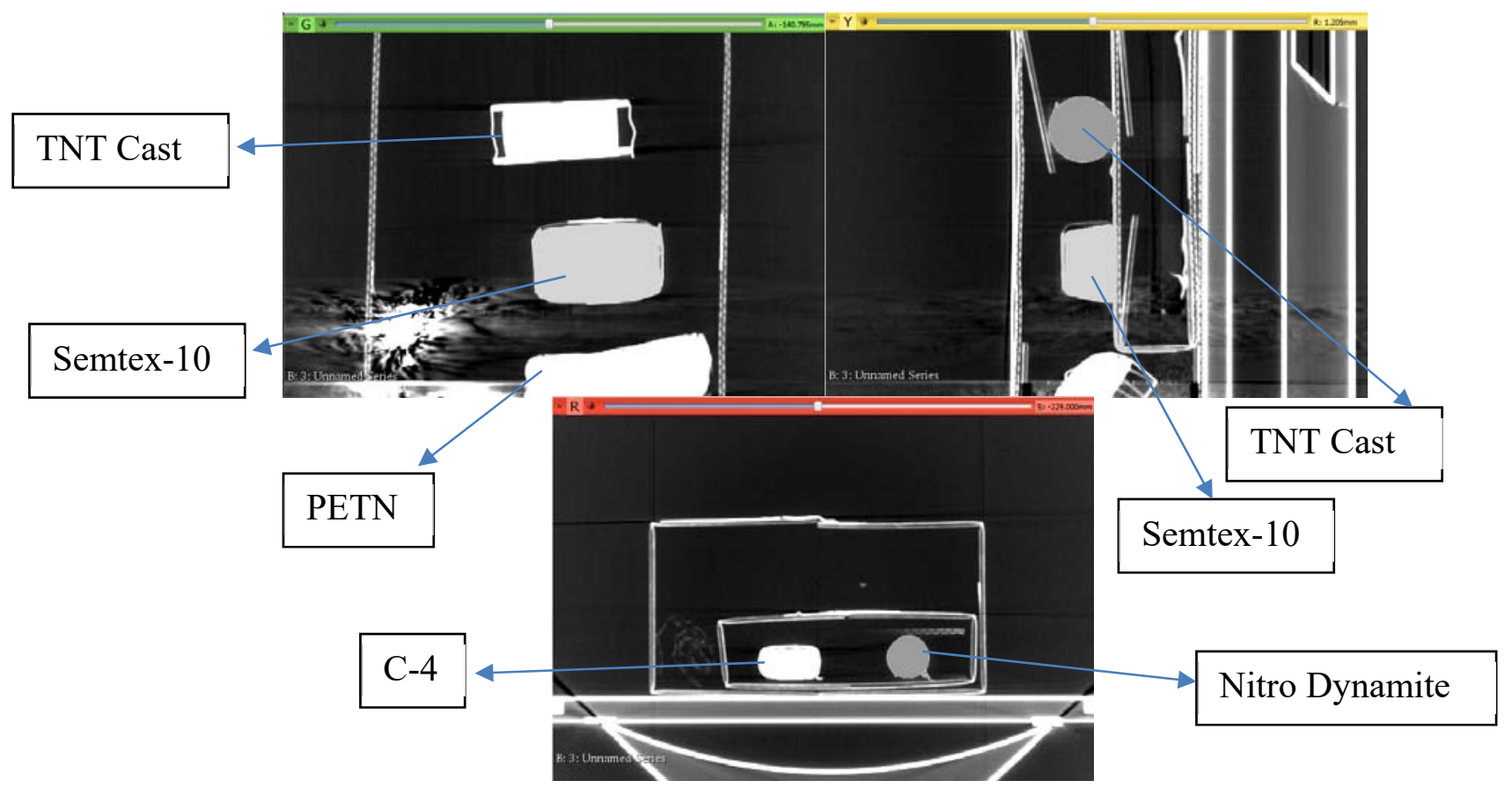

Figure 3.12 - Three orthogonal views of an image slice from 6 inert explosives.

We then correspond relative brightness to the density of inert explosives.

The values of the CT scan slice and respective densities are as follows:

\begin{tabular}{|c|c|c|}
\hline Inert Explosive & Density $\left(\mathrm{g} / \mathrm{cm}^{3}\right)$ & Value (in HSV color space) \\
\hline Semtex 10 Assembly & 1.43 & 0.9998 \\
\hline Nitro Dynamite & 1.6 & 0.9993 \\
\hline C4 Block & 1.58 & 0.8519 \\
\hline PETN & 1.77 & 0.6298 \\
\hline TNT Cast & 1.654 & 0.4214 \\
\hline Smokeless Black Powder & 0.95 & 0.3239 \\
\hline
\end{tabular}

Table 3.4 - The values obtained from the average of HSV pixel segment of each inert explosive. 
The next process is to evaluate relevant relative brightness values. If the density vs brightness value differs greatly from the distance of other density vs brightness value materials, we consider it an outlier and eliminate it from the results. As PETN and smokeless black powder are both forms of powder, pockets of air around the powder may have been captured and included in the segmentation process. These outliers are taken into account and removed from the final analysis.

We then compare the inert explosives' brightness to baseline brightness and normalize against the brightness values of the baseline. The lookup table is employed to calculate a relative density of an unknown mass, using the same parameters and scanner, with a brightness value obtained from the segment of pixels of the slice of the target.

Based on our model, an example evaluation would be a relative brightness value of 0.9 corresponding to an approximate relative density of $1.1162 \mathrm{~g} / \mathrm{cm}^{3}$ which falls under the material number most associated with densities that are associated with detergent or corn syrup, but not in the range of an inert TNT cast or nitro dynamite which should yield a brightness value of 0.9993 or 0.9998 . Table 3.4 is considered a proof of concept results table for use in determining a possible range of explosive. This table can be extended to include other inert explosives, and with the inclusion of multiple inert explosives scanned using this exact method, it may allow threat detection personnel to possibly identify the explosive, but this work is beyond the scope of this thesis.

We can map known densities to a photometric brightness value to a precision of $1 / 1000$, which suggests that as long as the scanner is accurate, we can map a large number of known substances to brightness values. 
Many techniques and approaches have been implemented in the medical field that use CT scanning to evaluate unknown masses in the human body. However, within threat-detection related fields, to our knowledge, no such methodology has been defined to determining relative densities of unknown substances in IEDs. By using inert explosives, the methodology we have presented is a theoretical baseline approach towards aiding EDU and other threat-detection personnel. This methodology may be quite useful if wide-scale adoption of portable CT scanners becomes more commonplace in the threat-detection fields.

We assert our claim that we are able to provide information regarding a range of the type of explosive(s) employed in the device and ascertain to a degree of certainty the nature of the unknown materials in a concealed container by leveraging the notion of evaluating brightness intensities to determine the relative density of an unknown material.

This section concludes the proposed methodology to calibrate a CT scanner and obtain a model to calculate relative densities as a form of additional information for threat-detection personnel. The algorithm however, does not necessarily depend on the use of a CT scanner, a 3D scanner such as an MRI scanner may follow the same protocol and map brightness values to densities.

In the next section we briefly discuss the creation of virtual models, cleanup and segmentation as this material is already published and discussed in our previous work [29]. 


\subsection{Serious Game Engine Model and Mesh Configuration}

\subsubsection{Virtual Model and Mesh Creation}

To create a 3D model from the DICOM data, we used a $3^{\text {rd }}$ party open-source software called "3D Slicer". 3D Slicer is used for the analysis (including registration and interactive segmentation) and visualization (including volume rendering) of medical images [76]. The software allows the user to visually select signal intensities to create and select label maps using

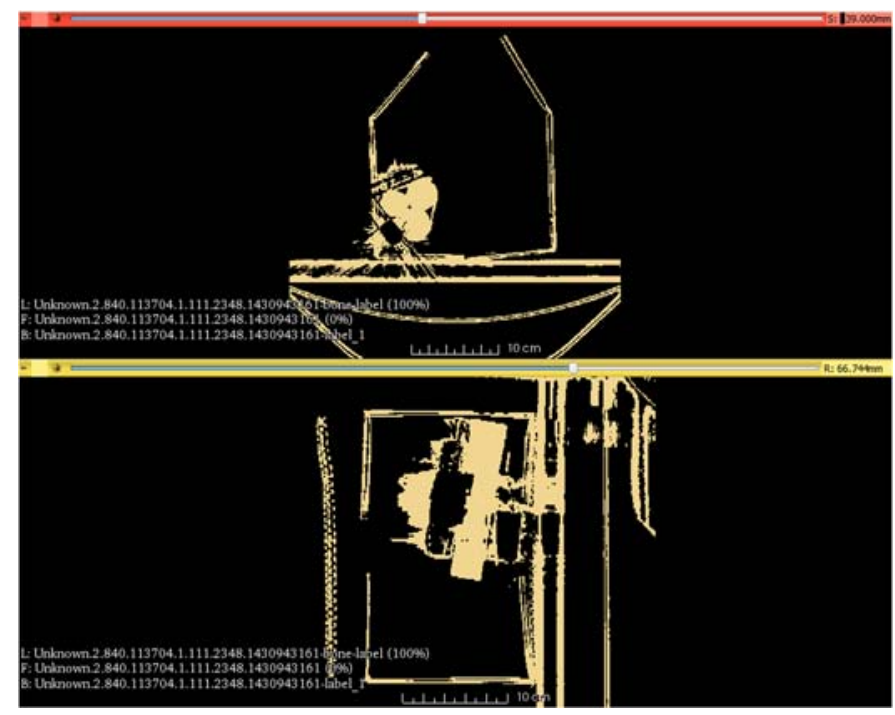

Figure 3.13 - A representation of a mock IED in a cross sectional views shown in 3D Slicer, these mock IEDs are previously discussed and labelled in [21], essentially the circles in the upper image are cross sections of wooden blocks.

threshold ranging. Essentially, the user selects a range of signal intensities, (or densities) that should be included in the 3D model. For our purposes, it is important to retain the exterior of the container in the 3D model. Since the exterior of the container is less dense than interior objects, it is difficult for the program to discern which parts of the image are relevant to the user (relatively low densities mean airspace), therefore the threshold feature in the editor allows the user to choose what entities should be merged into a model. 
However, the models created in 3D Slicer often include artifacts which are introduced by a variety of factors that may be related to a number of anomalies concerning the scanning like inadvertent motion, the presence of metallic components or foreign bodies being introduced [17]. Artifacts, as shown in Figure 3.14 and Figure 3.15, can be identified as dark streaks, thin bright and dark streaks that appear along the direction of greatest attenuation, or ring artifact which can be caused by low photon count (Poisson noise), beam hardening and scatter (dark streaks between two high attenuation objects (such as metal or bone) or suboptimal reconstruction algorithm [112].
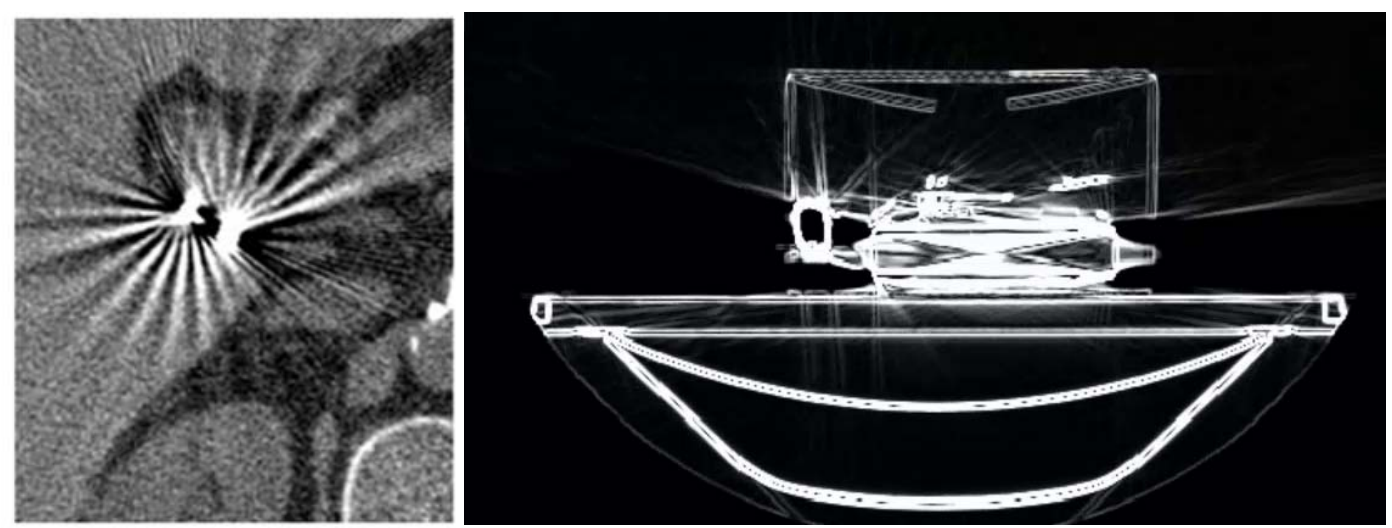

Figure 3.14 - Dark streaks or thin bright and dark streaks due to metal artifact that commonly ( $21 \%$ chance of occurrence in scans) appear in CT scanning due to the metal itself, or beam hardening, scatter effects and Poisson noise [17]. The scans are of a metal tube.

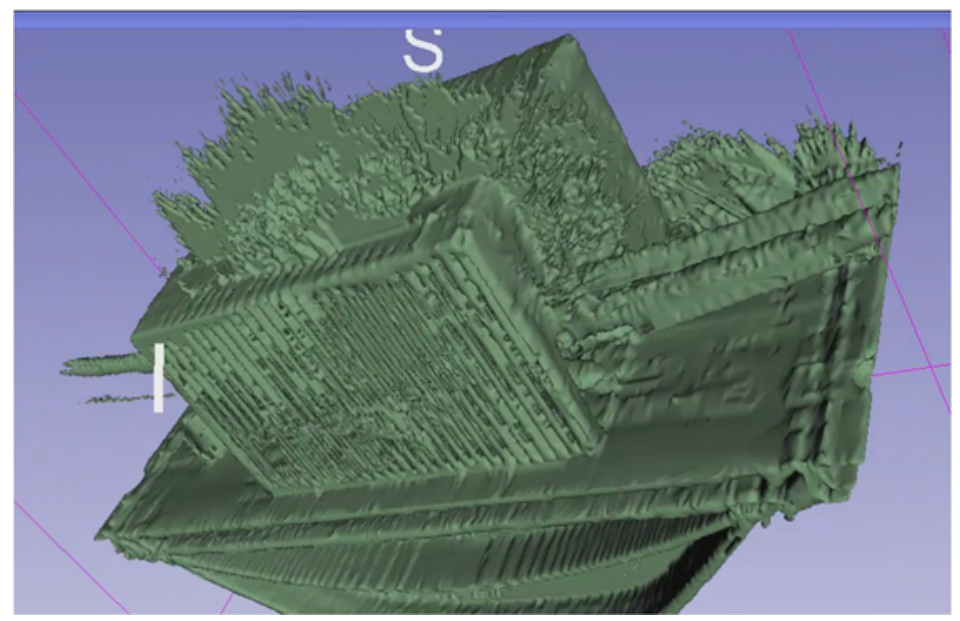

Figure 3.15 - Artifacts (streaks) that appear on 3D slicer after creating 3D virtual models from DICOM data. 
Closely observing the DICOM images, we notice that materials such as metal appear relatively closer to white than other materials such as wood, which appears relatively less close to white. This difference in brightness intensity represents the relative densities of materials in the image. It is important to note this fact because high density objects such as metal can easily create 'artifacts' in the DICOM image (a CT artifact refers to overflow marks or streaks that are not physically representative of the object - the use of MRI scanners commonly results in more artifacts [113]).

These artifacts are caused by the process by which CT reconstructs the object onto an image. This process involves a reconstruction algorithm employed in CT called the filtered backprojection (FBP) [114]. This algorithm suffers from disadvantages because projection data is measured by a finite number of detector channels using finite beam-width over a finite rotation interval. An x-ray tube source emits a continuous spectrum resulting in a phenomenon called beam hardening, and last, the measurements are often noisy and involve scattered radiation. The reconstruction of highly-attenuated objects such as metal causes streaking and star-shaped artifacts, which tend to obscure valuable details and reduce image quality [114]. Many approaches have been developed to reduce metal artifacts such as iterative methods and projection compensation methods. Compensation methods generate metal-free projection data and then reconstruct a metal-removed $\mathrm{CT}$ image, and iterative approaches base their approach on strict assumptions of the target scan. Iterative approaches are limited and become invalid once the assumptions are not met, and compensation methods may introduce new artifacts. Both are met with some limitations and disadvantages. However, further discussion of reduction of artifacts are beyond the scope of this thesis and will not be discussed. 
It can be concluded that high density materials such as metal will cause minor discrepancies in the image, which can possibly affect the appearance of the virtual model. The next section 3.3.2 will describe cleanup processes for these artifacts.

In additional to virtual models of IEDs, we also needed virtual models of EOD related objects such as a bomb suit, a disrupter cannon, and an EOD robot. Rather than using CT scanning for the models where we are only concerned about the structure and texture, we used a propriety software called Skanect 3D Scanning Software By Occipital [74] that uses a Red, Green Blue and Depth (RGB-D) structure-sensor.

An RGB-D camera has three sensors: one for capturing image data and the other two sensors are for capturing depth data. Depth data is collected using an IR projected-light stereo system and it is captured along with the image data to form a full colour 3D model of an object, a person or a room.

We use this technology to scan models such as the bomb suit, paintball gun, disrupter cannon, a EOD robot and its control box as well as any real-world environment up to a $10 \mathrm{~m}$ by $10 \mathrm{~m}$ by $10 \mathrm{~m}$ (length, width height) environment. The maximum size of the real-world environment is limited because of the structure sensor. The sensor uses infrared rays (IR) to project onto surfaces of the environment and triangulate distances from the surface to the sensor. These artificial IR rays are subject to infrared rays from sunlight, which means the structure sensor must be operated in an indoor environment or in outdoor environments at appropriate times of the day in which infrared rays from the sun are at minimal intensities (such as dawn or dusk, noting that scanning at night will not capture color/texture data). 
Any other $3 \mathrm{D}$ reconstruction software or structure sensor can be used as well to create virtual objects (and virtual environments) as long as the virtual objects are scaled, and programmed according to their intended real-world function, and tested and validated to a relatively high degree of fidelity. We chose to use this $3 \mathrm{D}$ reconstruction software as we only require a relatively accurate size and appearance of these objects for in-game user recognition in our serious game, so scanning from a CT scanner is deemed unnecessary for this purpose.

The process to scan these objects are relatively simple. Skanect is capable of using any RGB-D structure-sensor to scan any object in proper lighting, as long as the user follows the instructions to orbit the object being scanned. When Skanect captures data, it uses a stitching algorithm similar to StaticFusion [80], which reconstructs background environments using dense RGB-D Simultaneous Localization and Mapping (SLAM) [80] in dynamic environments. Skanect also provides algorithms to perform automated watertight mesh repairs, geometry simplification, precise model editing, and to export polygons to OBJ, PLY, STL and VRML file formats (all of which are commonly used file formats in 3D virtual modelling).

We use this technology to create $3 \mathrm{D}$ models of the simulation environment, explosive disposal robots, explosive disposal tools, and suspicious packages. Figure 3.16 shows models created from Skanect and used in our serious game simulation. 


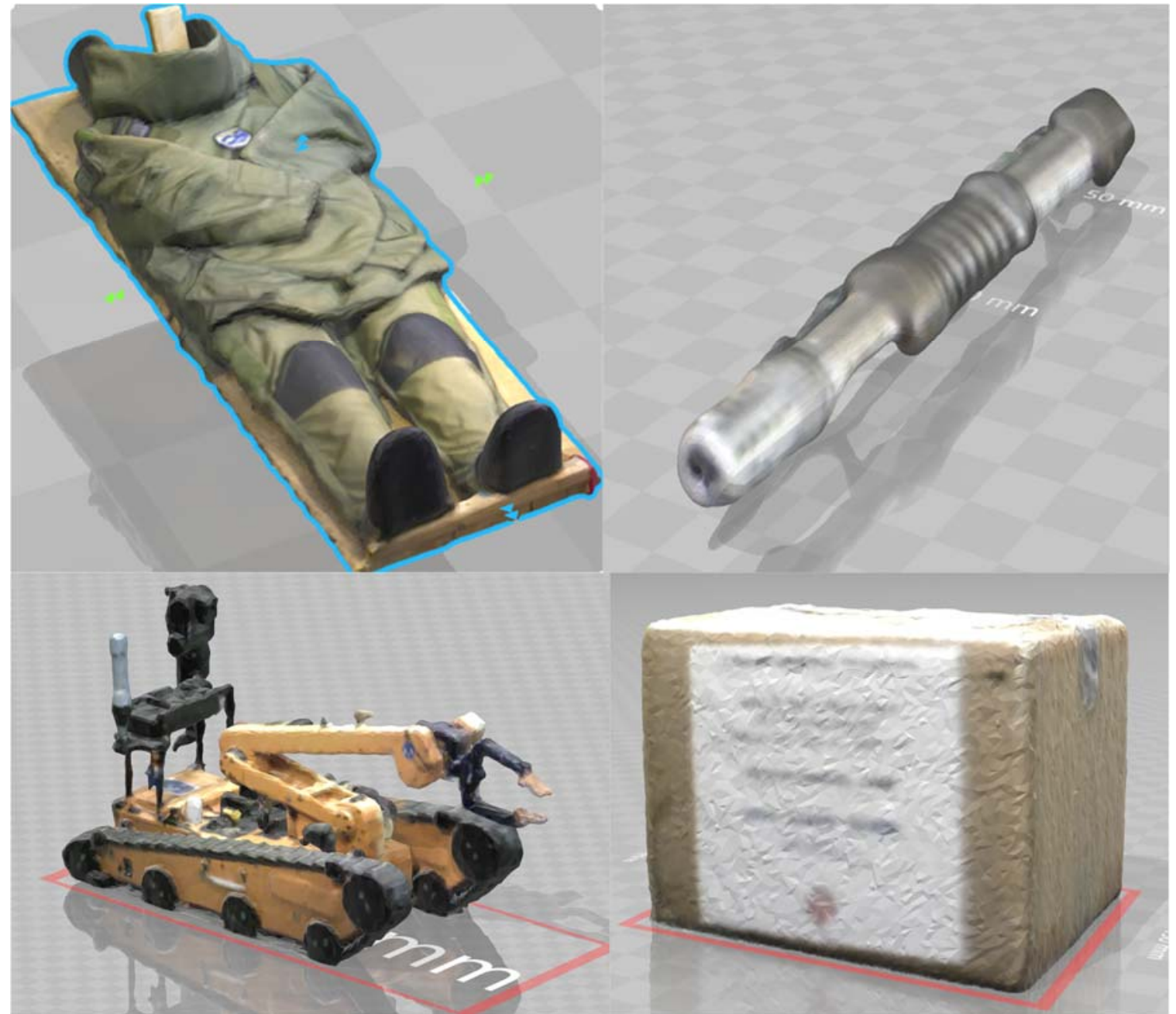

Figure 3.16 - 3D virtual models of EOD objects - top left: EDU bomb suit - top right: disrupter cannon - bottom left: EOD robot with camera and arm - bottom right: a suspicious package potentially containing an IED. 


\subsubsection{Model and Mesh Cleanup}

To cleanup artifacts and extraneous entities such as the support surface, we use Blender [88] to visually select and delete artifacts surrounding the mesh. Blender is an open source 3D graphics and animation software that includes a visualization algorithm containing a $\mathrm{C}++$ class library and numerous algorithms for scalar, vector, tensor and volumetric methods. It contains built-in advanced modeling techniques for implicit modeling, polygon reduction, mesh smoothing/cleaning, cutting and contouring. In our process, Blender is useful for visualizing the top, side and front view of the scene (often orthographic views) and this allows for visualizing protruding artifacts (dark and light streaks) that surround the containers.

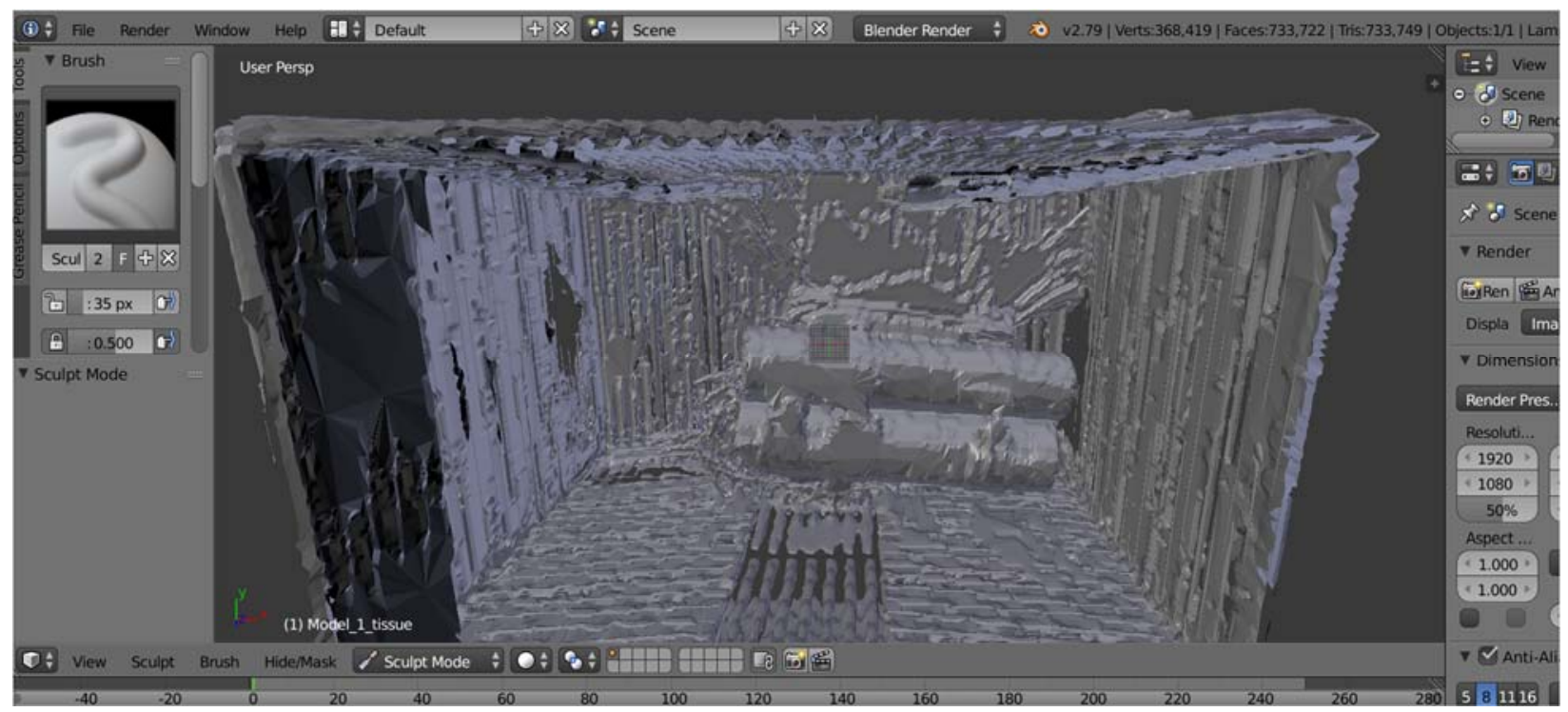

Figure 3.17 - A screenshot of blender viewing the interior of a container housing an IED, the front two cylindrical objects are virtual representations of wooden sticks simulating explosives.

We then use a hole filling algorithm [115] to generate a new surface mesh that follows the same shape as the input, but with more regular topology. The output of this algorithm is blocky re- 
meshes that are not too small or irregular. These meshes are useful for separating into components in Aperio [116], a 3D mesh occlusion viewer capable of segmenting meshes into parts.

The most important part of this process is to remove non-manifolds geometry. Nonmanifold geometry is essentially geometry that cannot exist in the real world [117]. Such geometries are described as 3 or more planes intersecting on an edge, a constraint that must be included when models are printed using 3D printers (printing of a described object cannot occur if it contains non-manifold geometry).
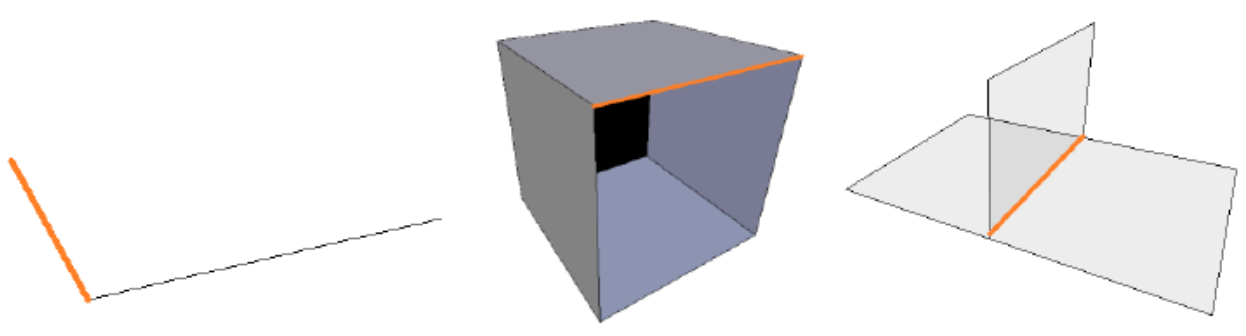

Figure 3.18 - These images describe non-manifold geometries which, in mesh topology, manifold objects are usually 2-manifold, and these geometries have edges that are connected by 0,1 or 3 faces.

This process removes small disconnected pieces and fills holes in the mesh and the resulting mesh appears much like Figure 3.19. 


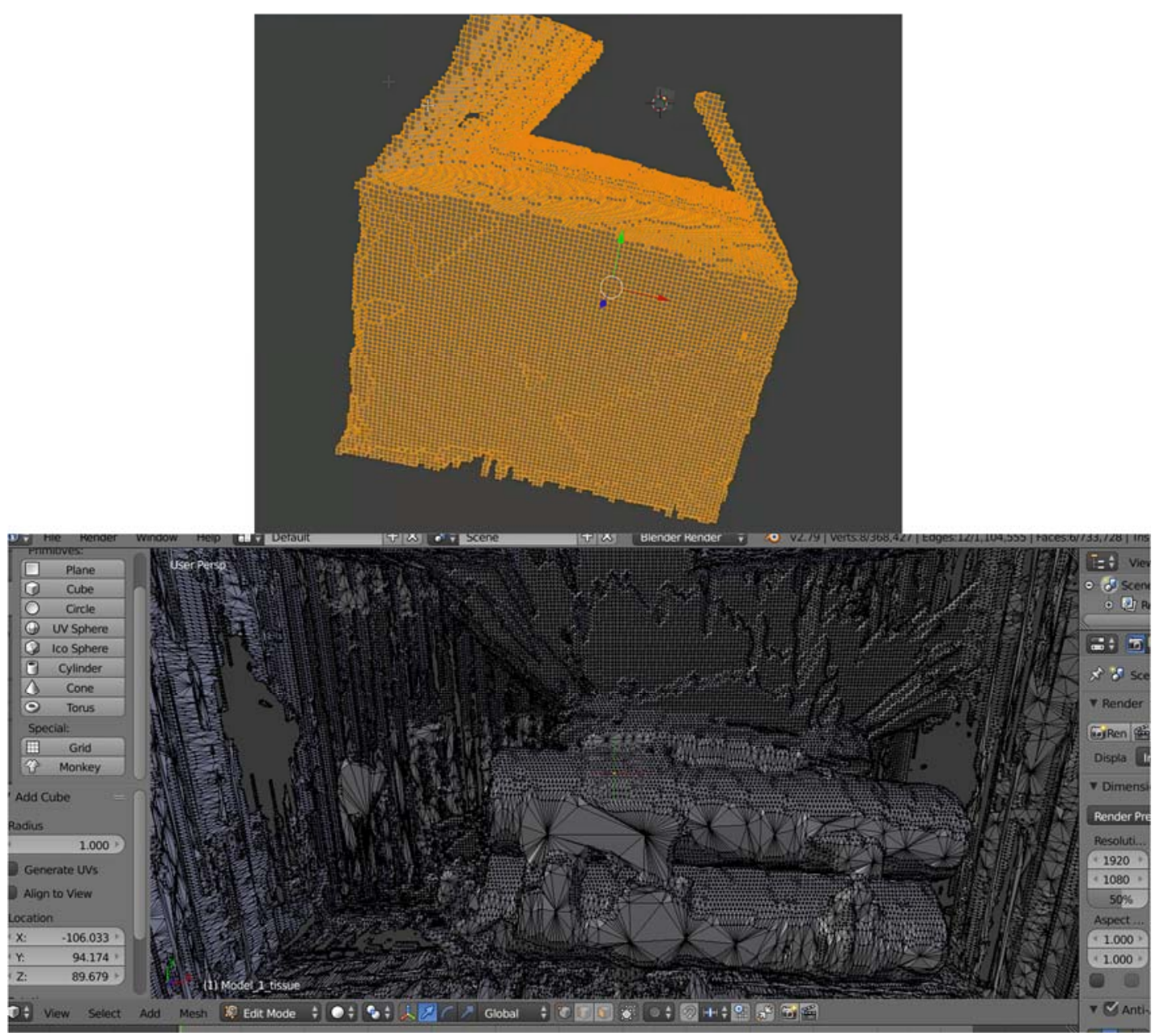

Figure 3.19 - The resulting mesh after the hole filling algorithm is applied - all non-manifold geometry are excluded.

We export the mesh as an object file which is compatible for import into Aperio [116]. Aperio is a novel interaction model for managing 3D scene occlusion and for visualizing and understanding spatial relationships among parts of a multi-part mesh-based system. 


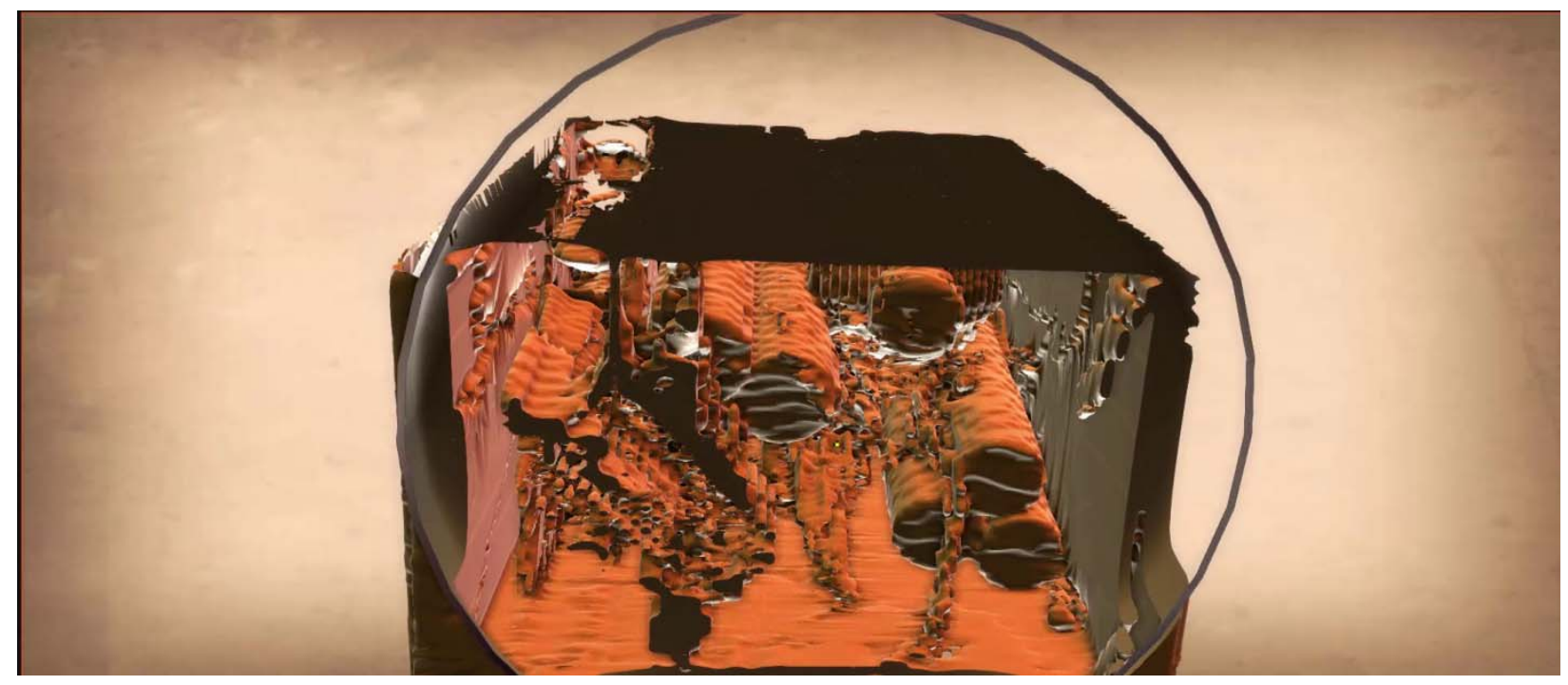

Figure 3.20 - A screenshot of Aperio. The container is modified using a mechanical tool to reveal the interior and to separate critical IED components of interest.

\subsubsection{Component Recognition and Segmentation}

Aperio [116], a 3D interactive visualization tool developed for medical image analysis which allows for the use of segmentation tools similar to real-world mechanical tools. These virtual tools allow a user to view relationships between structures that are often layered and occlude one another (a crucial element in the EDU process for examining suspect IEDs). The program manipulates the mesh with 4 tools; a cutter, ring, rod and knife tool.

The ring and rod tools are used for translation and rotation of meshes. The ring tool allows meshes to slide along its circular path similar to how beads move on a wire, and the rod tool allows meshes to slide along its linear path. These meshes can also be spread apart along the ring or rod to create exploded views of mesh parts, and they can also be spun around the rod's axis or fanned apart. The ring and rod tools are presented as an alternative interaction technique for creating 
exploded views of mesh data along a constrained path, (rather than freely exploding meshes in every possible direction like traditional radial explosions). These segmented parts can also be reversed (restored) by simply moving a GUI slider (along with the connected meshes) back to their initial rest positions.

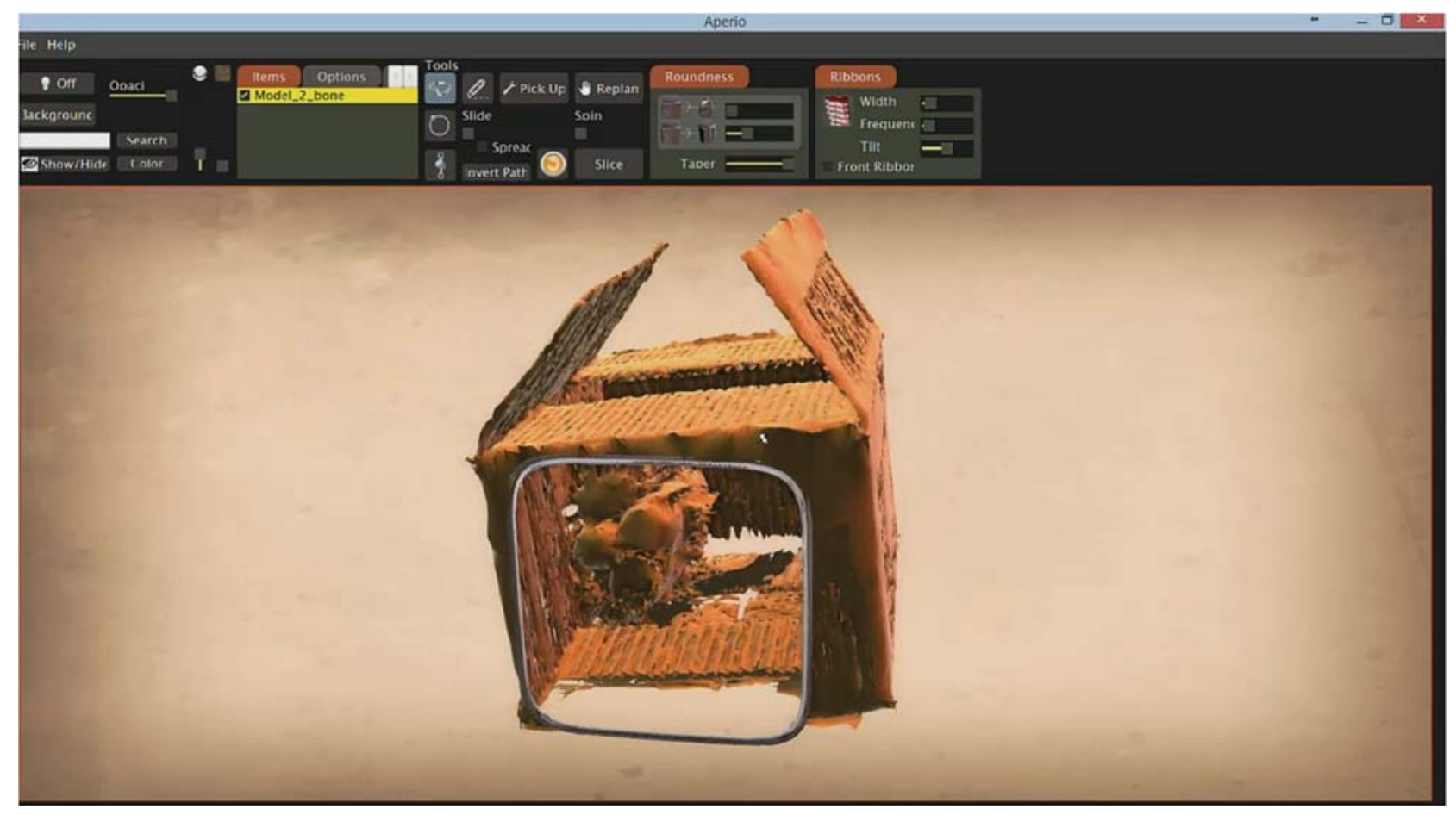

Figure 3.21 - The mesh shown in Aperio after using a cutter to view objects that was occluded by the container.

At this point in the process, EDU specialists [118], [119] identify which parts of the mesh visually represent critical IED components and separate them using these tools. The output is an object and material file compatible for importing into a game engine. 


\subsubsection{Component Bounding Box Association}

After importing the assemblage of components into the game engine, Unity [90], we associate bounding boxes to these components and assign unique scoring criteria to each box. The bounding box regions indicate the 4 core components of an IED (power source, initiator, explosive, and switch) which are verified by an EDU specialist [102], and a weighted score is assigned to each region.

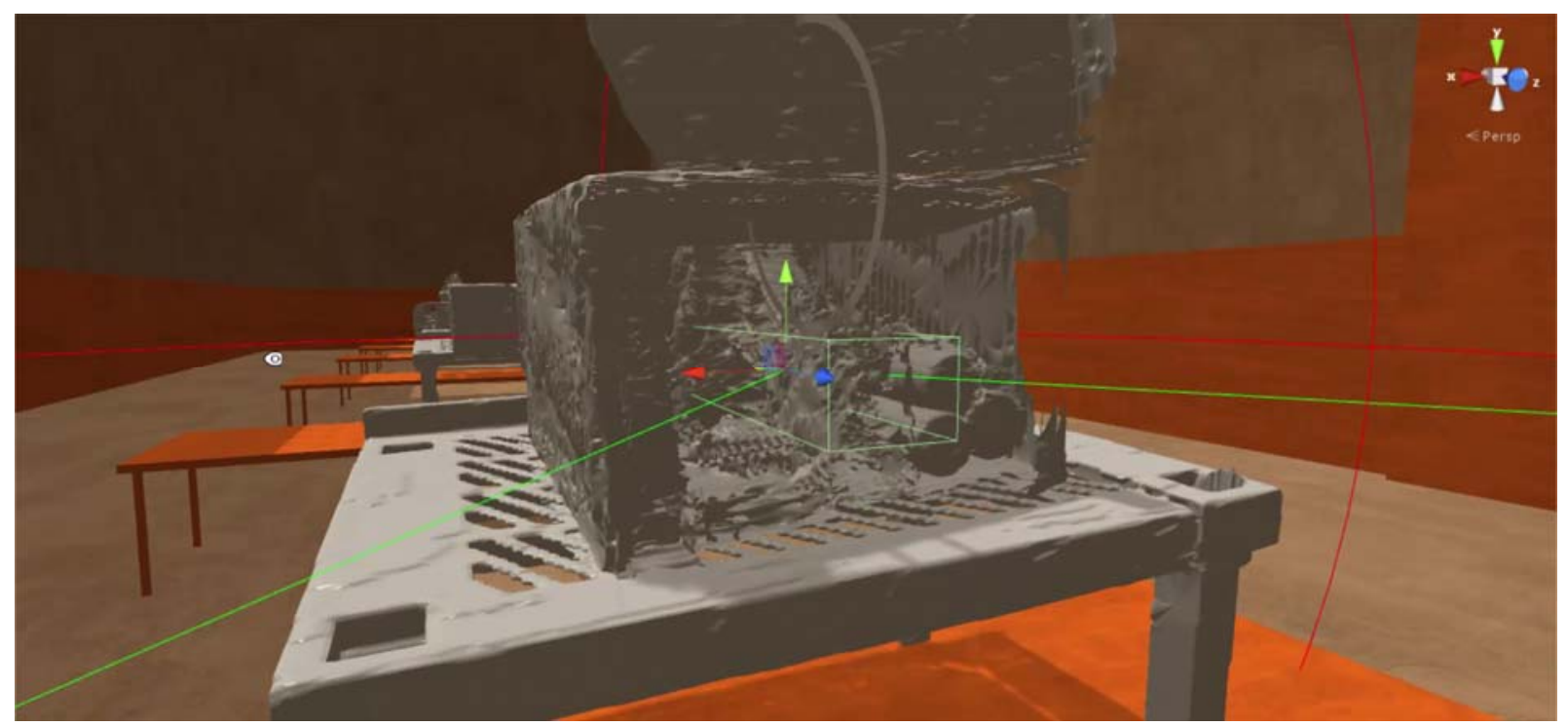

Figure 3.22 - A bounding box assigned to a critical component in the simulation.

The algorithm for the bounding box has three scripts ${ }^{2}$ attached - a damage receiver script, a bullet penetration script and a crosshair script.

The damage receiver script, attached to the bounding boxes, function as a trigger that indicates the damage applied to the component and the value of the damage received from the projectile fired from the player's weapon-in our case, the disrupter. This allows the script to

${ }^{2}$ A script is a program written in $\mathrm{CH}$. 
calculate the current status of the bomb; neutralized, not neutralized yet, or exploded (signifying the game ending).

Information on the scripts are explained in our previous work [29] and can be found in the appendices.

\subsection{Physics Engine Model Configuration}

\subsubsection{Molecular Dynamics Implementation}

This section discuss the methodology we developed that disintegrates 3D models into particles, with each particle programmed with characteristics of its real-world analog and is subject to 3-dimensional physics when external forces are applied.

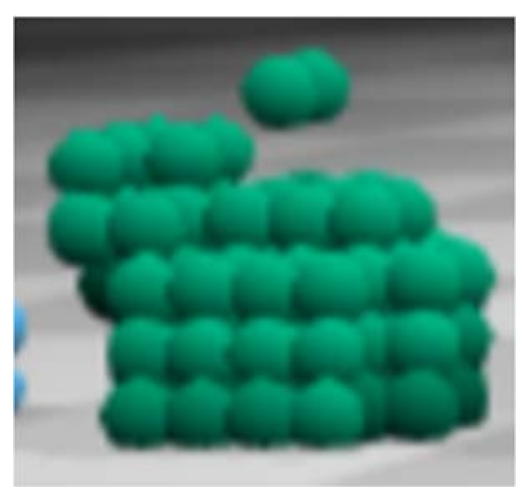

Figure 3.23 - A representation of object disintegration into finite elements [18].

We disintegrate each component of an IED model by creating mesh chunks out of each component. Each chunk is considered as a particle and is represented as a game object.

The input parameters to the mesh chunk function are user input width, length and splits, where length and width are the length and width of the entire mesh and splits represents the number 
of times the mesh is divided. The width and length vector information are then added to vector lists containing the vertices and triangles of the particles.

The script is shown in Appendix B. The algorithm takes 3 inputs as parameters - width, length, and splits, and the algorithm is as follow:

1. Create a new mesh with dimensions given by the split value.

2. Create new mesh chunk, and lists for vector and UV mapping data.

3. Iterate through each chunk length and chunk width, divide the original mesh's vertices and UV mapping according to the parameter width.

4. Add the new vertices and UV mapping to the vector lists

5. For each length, divide the chunk width according to parameter length, and divide the original mesh's vertices and UV mapping according to the parameter length.

6. Combine the lists and recalculate bounds and normal vectors.

7. Return the mesh containing chunks.

The value for splits decides the number of particles, the higher the split value, the more accurate and computationally expensive the simulation is. The arbitrary number 6 shown in the script is due to the current game object limitation of Unity. One IED model is split to approximately 4000 particles.

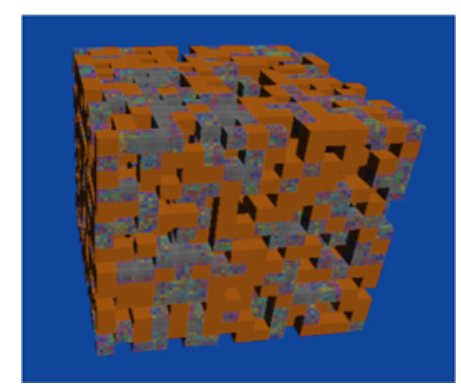

Figure 3.24 - A magnified view from a Unity scene of multiple game objects after the mesh chunk algorithm is applied. 
We then remodel exterior particles to appear as it's a seamless mesh. The exterior of the model is compared to the original mesh. Each exterior particle is reshaped to match the original mesh's exterior, and their vertices and triangle information are modified accordingly in the vector lists. The remodeling algorithm is as follows:

1. Superimpose the original mesh onto the particle mesh.

2. Maintain only the surface mesh information of the original mesh.

3. Apply the material, meta and png data onto the superimposed mesh.

4. Remove gravity constraints to all the game components of this mesh and particles.

5. Remove material mesh (so particles can move freely) and reenable gravity constraint upon projectile impact.

Particles are considered strictly non-spherical, and most of the exterior particles have no uniform shape, so that the overall model retains a relatively seamless structure rather than a blocklike structure.

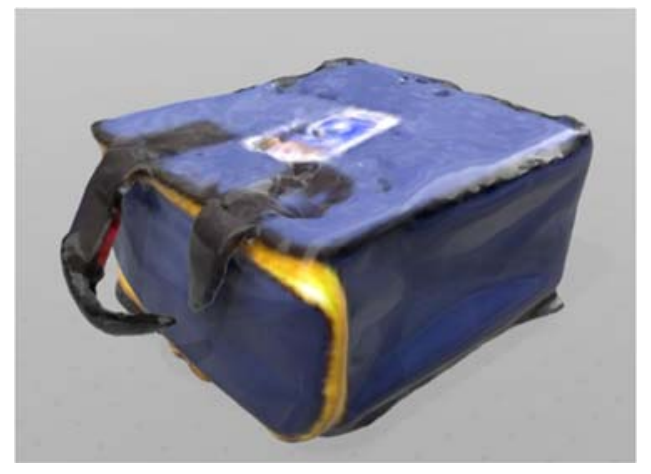

Figure 3.25 - A reintegrated form of a 3D scanned model bag containing inert explosives.

In this reintegrated form, the particles remain in persistent contact, the texture map is reapplied only for visual effect. These particles are programmed to stay as a rigid object until an external force is applied from a projectile. 


\subsubsection{Real-world Physical Attribute Implementation}

For each particle of a disintegrated mesh of an object, we associate the calculated relative density value from our data analysis of our CT image, and its estimated volume (which is volumetrically determined through $3 \mathrm{D}$ virtual model information).

We utilize the relative density measurement obtained in our data acquisition and the volume of the target object. Since, we have the equation for calculating mass:

$$
m=d V
$$

where $\mathrm{m}$ is mass $(\mathrm{g}), \mathrm{d}$ is density $\left(\mathrm{g} / \mathrm{cm}^{3}\right)$ and $\mathrm{V}$ is volume $\left(\mathrm{cm}^{3}\right)$. With the calculated mass, we can apply Newton's second law of motion in the cartesian coordinates (x,y and z), when it is subject to an acceleration to obtain force in cartesian coordinates:

$$
f=m a
$$

In the update function, it's physical movement is computed as the sum of its interactions from external contact forces, which result from any number of particle's velocity, mass, and acceleration.

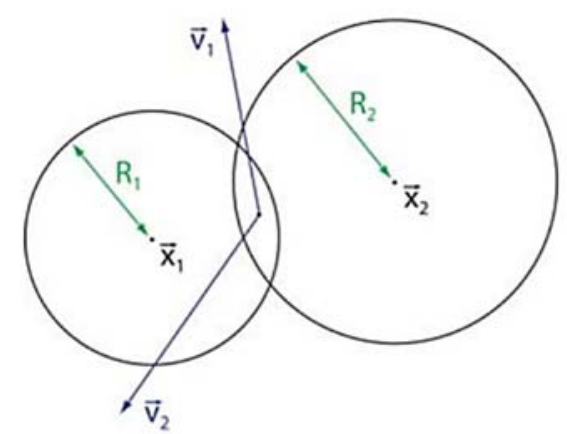

Figure 3.26 - This diagram represents the Newtonian collision interaction between two particles and the elastic deformation represented by the small overlap between particles. 
As an external force from a particle is applied, the motion of the particle and the collision with other particles is modelled at each update. The forces described here are applied to the centre of the particle. Equal and opposite forces are also applied to other particles during collisions.

These particles are also subject to gravity and an energy loss function. More factors and variables may be implemented in the future and the algorithm allows for any additional forces as long as the resulting forces can be added or subtracted to current forces (having properly adjusted vector directions).

\subsubsection{Energy Loss Function}

As particles collide with each other, they are subject to particle stiffness and particle dissipation [120], also known as the viscous damping coefficient and elastic restoration coefficient [121]. These coefficients are multiplied along with the applied directional forces to simulate force dampening as a result of energy loss.

These coefficients are not known and must be chosen to match experimental data, or manually adjusted to match a desired response. According to [14], the model that best agrees with experimental results is particle stiffness at $1 / 2$ and particle dissipation at $3 / 2$ at collision.

\subsubsection{Shear Forces}

We also added shear forces to each particle, which are computed as a displacement when particles make initial contact. This shear force has a friction coefficient and normal force that will appear only as a slowing effect in the tangent direction. 


\subsubsection{Projectile Implementation}

For each projectile instantiated, we associated fixed density, mass and volume. These attributes are known values, and are dependent on the type of projectile used in the disrupter. These values should differ from the relative density values for the suspect IED made of unknown materials. This is understandably problematic. We approach the problem somewhat unrealistically by obtaining relative densities via CT scanner technology. Each shot fired is simulated through a customized program that incorporates acceleration, mass, velocity and computed forces. The projectile is assumed to be spherical. In addition, more than one projectile can be shot at the same time.

To account for gravity, terminal velocity, air resistance, and mass, we utilize the equation of motion of the projectile:

$$
m\left(\frac{d v}{d t}\right)=m g-c v
$$

where

$$
v=(v x, v y, v z)
$$

is the projectile velocity, $g$ is the acceleration due to gravity and $c$ is a positive constant, which is part of the terminal velocity, so $c v$ can be considered the dampening force.

At each update from the point where the projectile moves from the model cannon barrel to the target, the muzzle velocity is subject to deceleration with a dampening function. The dampening function is calculated by

$$
R=\frac{v_{o} v_{t} \cos \theta}{g}
$$


In equation $5, r$ is the horizontal range, which is a function of velocity at time 0 , to velocity at time $\mathrm{t}$ multiplied by the angle above the horizontal plane. Angle 0 would mean a shot is horizontal to the floor, and has no resistance (dampening). These equations are follow concepts from projectile motion with air resistance [122].

The projectile script also includes the implementation of a scoring function, where the users score increases whenever they have effectively shot a projectile (simulates shooting an EDU disrupter) onto a correct component (simulates hitting a critical component, for example power source, trigger etc...). The score decreases whenever the user misses a correct component, but also misses a lethal component (which will end the game).

The bullet penetration script outputs projectile information to the user per shot fired. If the bomb is not triggered to explode and the component has been effectively hit, the program will output the distance the user's weapon is to the target and 3 directional angles which are calculated from the 3 orthogonal planes (XY, YZ, and ZX plane) at the point of the barrel upon firing. This information is obtained from reasoning within the simulation and can be useful for EDU disrupter positioning processes.

The crosshair script allows the user to see whether the crosshair is in the range of a component, correct or lethal. It is meant as an aid for the user to recognize which parts of the IED are associated with a bounding box (key component of interest). The crosshairs are reality-based in that they allow aiming with the game in a similar way as aiming would occur in reality. 
Aiming the disrupter in the game provides additional information about the contents of the IED package which would not be readily available at a real incident. It is reasoned that this additional functionality can be used to assist in the neutralization process, and as players of the game get better at aiming at IED components, less information would be revealed by the crosshair tool. However, this is a matter of speculation and the use of supplemental crosshair data is beyond the scope of this thesis.

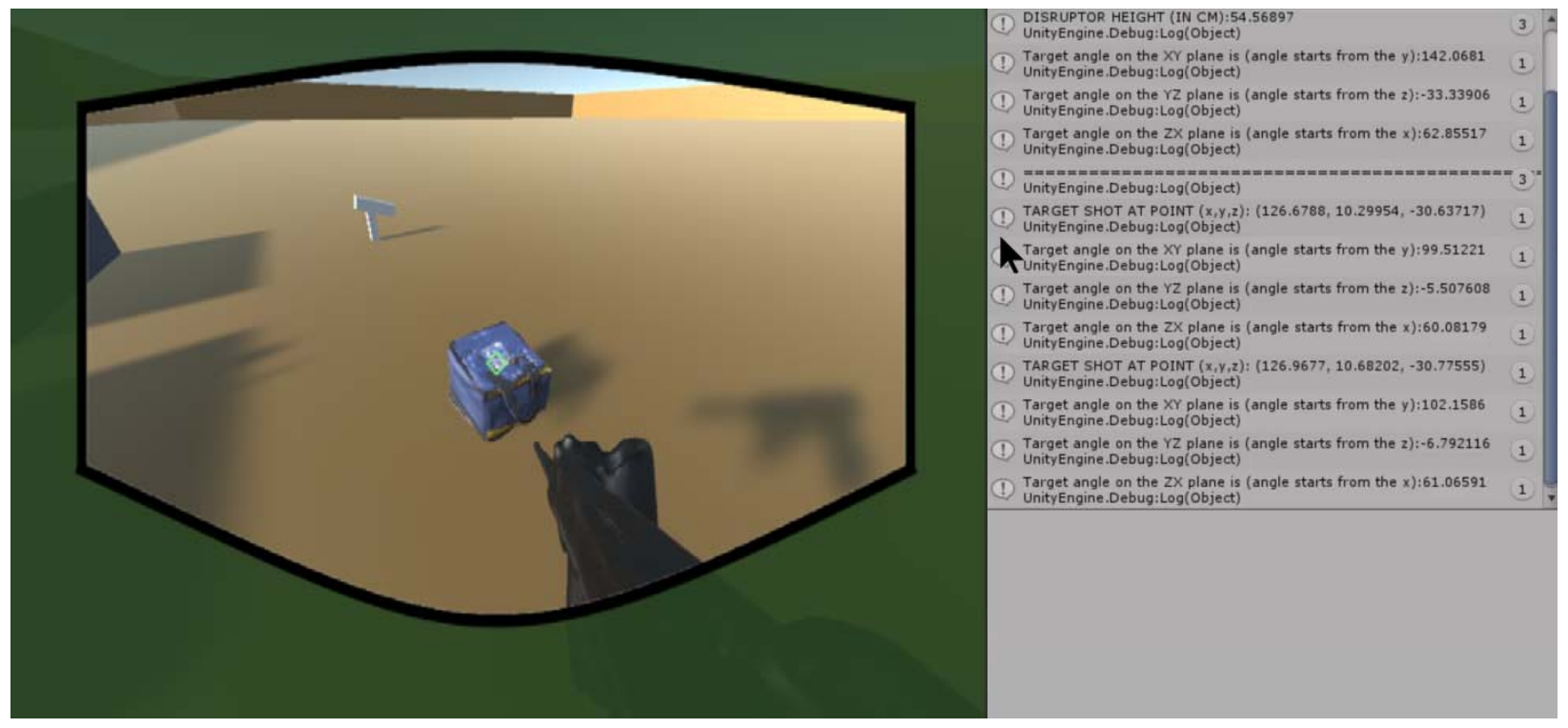

Figure 3.27 - A scene in the simulation after firing the disrupter at components.

\subsubsection{Dispersion Model Implementation}

In our simulation, particles are represented as game objects, equipped with mesh tight bounding boxes. We assume that if any of the game objects collides with the $3 \mathrm{D}$ terrain, then it is considered a significant point of impact. The particles moves in the simulation according to userdefined density, and volume, and in-game calculation of force and directional values and are configured to detect collisions with the mesh of the 3D terrain. We assert that the point of collision with the 3D terrain suggest a likely point of impact in a real-world environment. 
After a projectile impacts an IED, a $10 \mathrm{~cm} \times 10 \mathrm{~cm}$ bounding box is provided at the target as a potential visual indication of particle impact. Caution should be used in the interpretation of the marked bounding box, as it may not necessarily encompass all particle surface impacts in the real-world. We verify potential impact points with actual real-world evaluation in the next chapter.
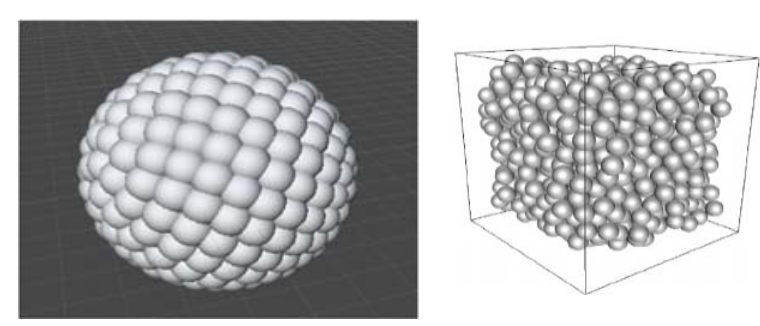

Figure 3.28 - Multiple spherical unity game objects that are grouped together representing shrapnel.

Game objects are grouped together representing an IED. Figure 3.28 shows multiple game objects grouped together representing shrapnel. This group of game objects are placed within an IED matching its real-world placement of shrapnel.

No actual model is in place to simulate an explosion, but any projectile's primary impact point is an explosive within the IED. In this case, the simulation will indicate to the user that the neutralization attempt is unsuccessful and the IED has most likely exploded and the game will end. The entirety of these game objects are placed together as an IED (with accurate representations of its configuration) and move in the simulation as a unit.

Within the IED, there are also empty pockets of space. These spaces are the reason why molecular dynamics is useful. The simulation is capable of modelling a projectile's impact onto particles and subsequent accurate particle displacement and its passage through pockets of air and collision with additional particles. This phenomena is difficult to model in continuous mechanics 
and is known to be better represented using molecular dynamics [14]. However, it should be noted that all factors that are present in a real-world situation are not modelled in this serious game simulation. The implementation of all possible factors in a virtual simulation as an open system is not yet computationally feasible and is out of the scope of this thesis (for example, as of now, it already takes 1 second to update each frame for our current simulation upon projectile impact on an IED). This simulation however, does provide a proof of concept for our algorithm of an IED neutralization serious game.

\subsubsection{Game Actor Implementation}

Inside the game, the player has a choice of picking up different models of disrupters. Each disrupter has a specific projectile that has a unique velocity and volume. The higher the velocity, the greater the penetration power and perhaps the farther the materials will disperse, subject to firing angles and in-game physics. The assumption of the simulation is that the neutralization happens indoors, and no other external environmental factors affect the particle dispersion physics.

The objective of the game is to aim and shoot a projectile to neutralize the IED. As briefly described in the previous section, as the projectile hits the object and its bounding box, an effectiveness score appears indicating whether it has successfully hit a key component or not. Additional information such as splatter impact points from a resultant projectile is also marked on the scene and spatial coordinates are output on the console display.

The player must look for visual cues shown within the bomb model. As the player hovers the crosshair near key components with the disrupter, the crosshair changes color, and as the projectile hits the bounding box, the distance and the angle to the target with respect to the normal plane of the disrupter are shown. 
If the neutralization shot is successful, then the virtual IED will disperse according to the force of the projectile upon impact, and the user's score will increase. If the neutralization shot misses the critical components but does not hit an explosive component, then the user can attempt to neutralize the virtual IED again, and the user's score will decrease. If the neutralization shot hits the explosive component, then the user has essentially detonated the virtual IED, and the simulation ends, and no explosion is shown.

There may be cases where runtime errors may occur, but as of now, the simulation does not encounter any fatal error that will force the simulation to end abruptly. When any such error occurs, Unity has a built in automatic descriptive error message that outputs in the error tab of the console. The dispersion process uses customized impact scripts that calculate the affected particle's trajectory and move these particles in a simulated direction. These particle's velocity are subject to energy loss functions and shear forces with user-defined coefficients. 


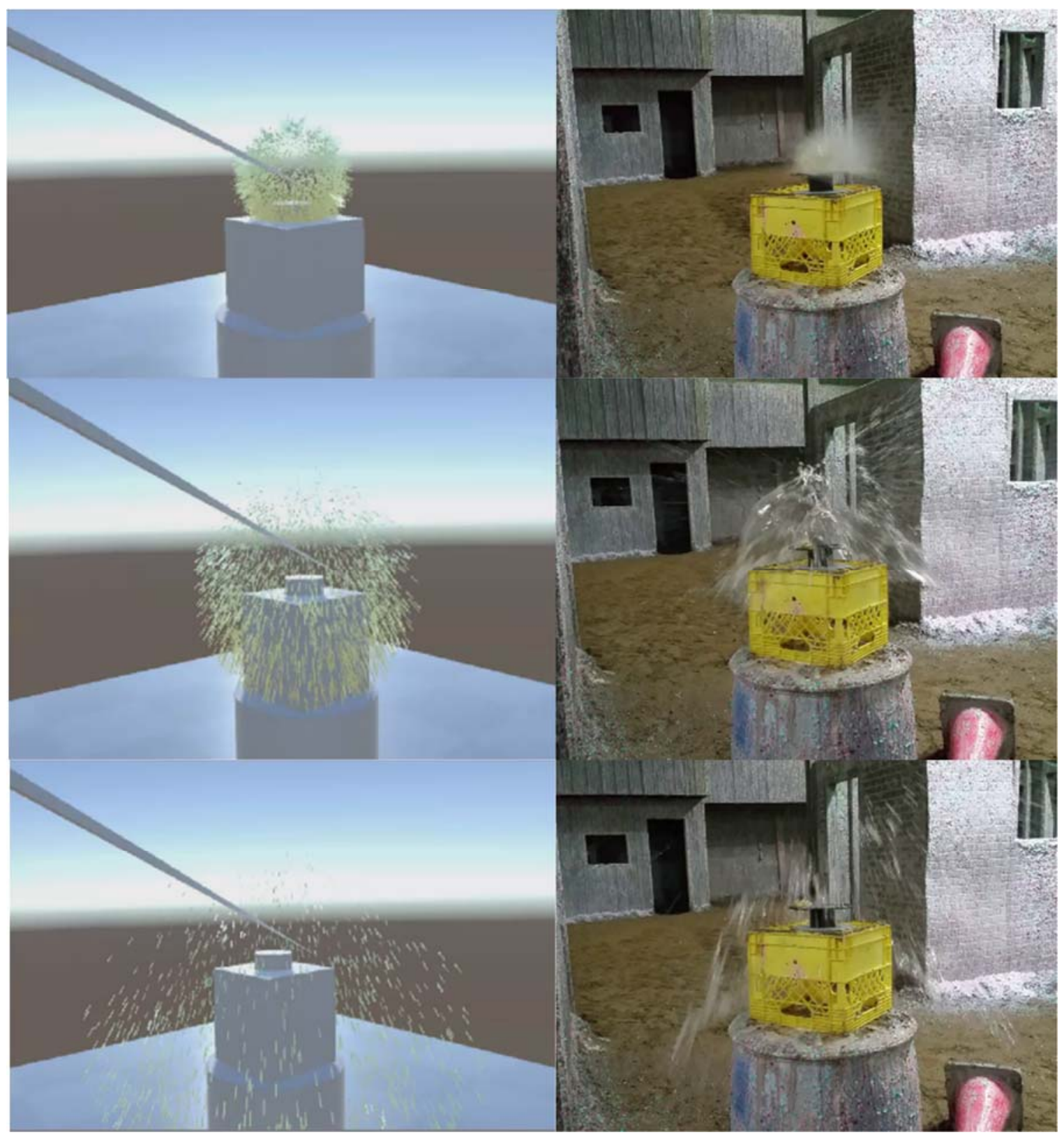

Figure 3.29 - Left: Simulation of a particle dispersion in action. Right: Real-world experiment of projectile impact resulting in dispersion.

To measure the effectiveness of the disrupter's target location with a projectile blast in the game, we take advantage of known heuristics. For example, if the disrupter fails to hit the desired target the score will be low, while hitting the target leads to a high score. Of course, hitting the 
main charge causes the game to notify the actor that an explosive has been hit (neutralization is unsuccessful) and the game will end.

The simulation provides feedback to a player through scoring points as to the effectiveness of their targeting. We suggest that this interactive part of the simulation can be used to safely train response personnel by providing visual cues and feedback for important and specific regions of interest of IEDs.

\subsubsection{Pre-neutralization Investigation Mode}

The game actor is configured to be able to pick up and wear a bomb suit, control a EDU robot, and place a disrupter aimed at any point in the virtual environment. Real-time geospatial information such as the game actor's pose, and the disrupter's pose in cartesian coordinates that are mappable to the real-world is provided on a console display for the user to accurately place the disrupter at a specific point in the virtual environment. The user can activate the disrupter and upon successful neutralization, the subsequent dispersal of particles which will automatically compute and simulate their trajectories and possible collision points with the mesh of the $3 \mathrm{D}$ environment. 


\subsubsection{Post-neutralization Exploration Mode}

After a successful IED neutralization occurs, users are able to move the game actor anywhere in the virtual environment to analyze and view the impact points more closely. All the impact points are also recorded as a list of coordinates.

The simulation also indicates the spatial grid that have the most impact points. The dispersion splatter is measured with a 5 by 5 sensor grid pad with each pad measuring $81 / 2 \times 11$ inches which we will use to validate splatter impact points in the real-world in Chapter 4 experiments. Depending on the forces applied to the particles, the simulation computes the particle's destination and outputs onto a console the percentages that each grid has been affected.

For example, the simulation may indicate that grid section A5 and B4 have most impact points.

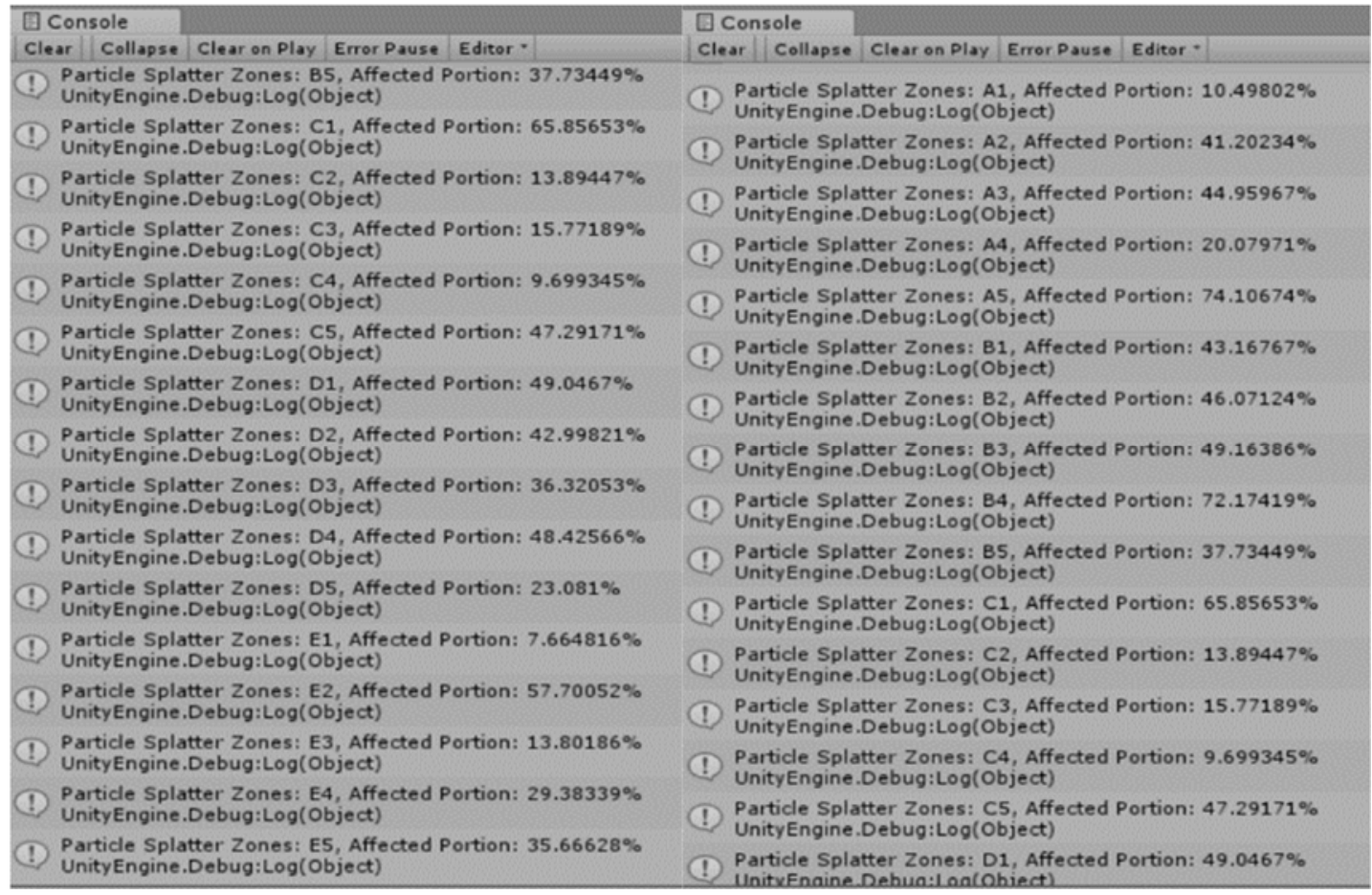

Figure 3.30 - Simulation console indicating the percentage of a sensor grid affected by particles. 


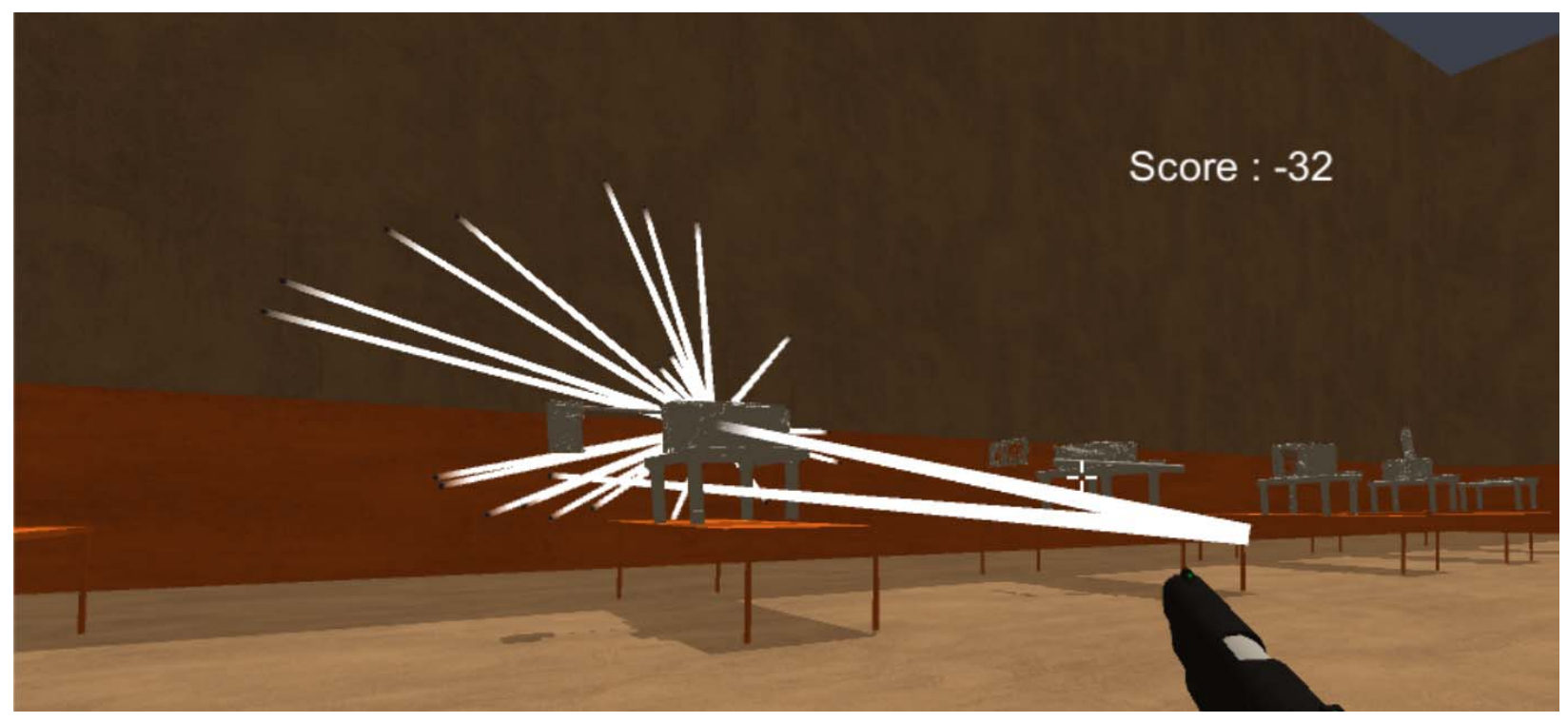

Figure 3.31 - Exploration of the possible dispersion area and points of impact of particles after a successful neutralization of an IED occurs.

Our focus is on identifying the exact location of critical components within a concealed suspicious package and ensuring the assemblage of these components are accurately represented within a game simulation.

The focus of the evaluation which will be discussed in chapter 4 is to compare the 3D model inside the simulation to the real-world and determine the degree of fidelity. It will also describe the remainder of the algorithm which includes evaluation of the density measurements of unknown objects, and testing the accuracy of the simulation's splatter impact points. 


\section{Chapter 4 Experimental Results}

\subsection{Introduction}

The main objective of our research is to devise an algorithm that can be used to create an IED neutralization serious game simulation for the purposes of augmenting EDU processes.

Towards this main objective, our algorithm addresses several sub-objectives:

1. Provide a physically accurate representation of an IED that maintains all spatial relationships between its components. (supports contribution 1,2 and 4)

2. Provide information regarding a range of the type of explosive(s) employed in the device and ascertain to a degree of certainty the nature of the unknown materials in a concealed container. (supports contribution 3)

3. Provide splatter impact points and primary and secondary effects on an IED's surroundings as a result of an effective neutralization shot. (supports contribution 6)

4. Provide potentially actionable intelligence obtained from 1,2 and 3 to EDU - information such as objective scoring of planned actions, neutralization cannon pose and direction, reasoned density estimate of unknown occluded target objects, and splatter impact points on the surrounding environment. (supports contribution 5)

Thus, the basis for the argument that our thesis research has been successful is the evidence that our algorithm can provide accurate 3D models of IEDs, provide relevant information regarding the possible type of explosive employed in the device, and provide accurate splatter impact points as a result of a successful neutralization shot - all within a game simulation that, 
in turn, supports the ability of EDU to make intelligent decisions concerning how they should safely handle real explosive devices based on accurate interactions with simulated surrogates.

This chapter discusses the results obtained from our validation methods, which we claim, while not a comprehensive validation of all functionality within our system, are necessary in verifying our approach and providing a viable justification that our algorithm can create a relevant serious game. This portion of the algorithm validating the model and splatter and test the serious game is shown in Figure 4.1.

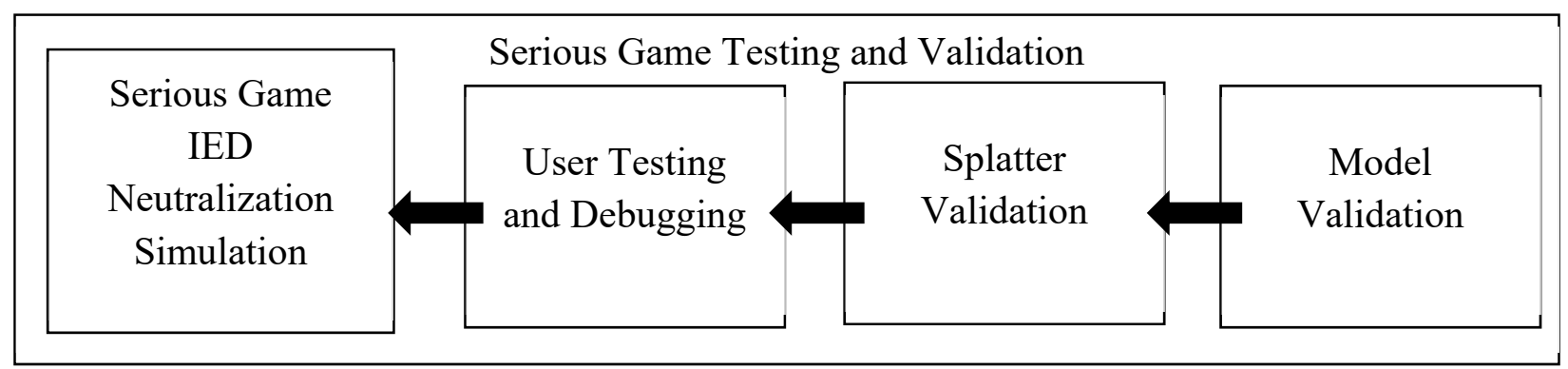

Figure 4.1 - The algorithm's last phase: validate the model and functionality and also to test the serious game.

These tests are intended to assess the validity of our algorithm, the system resulting from it, and provide weight of evidence that the system can be used to support real-world EDU decisionmaking processes based on the virtual objects that can be represented and interacted with.

Because of the dangerous and unpredictable nature of IED-related events, experiments are only performed in simulation or at purpose-built, bomb disposal ranges or designated areas. We make the assumption that our claim of a valid simulation for analysis in neutralization processes in general based on the practices of EDU professionals in North America. We are not making the claim that this system can be used in all circumstances. Nor do we claim that our method of sensing can be easily applied. However, our analysis seeks to support the assertion that this algorithm and 
consequent systems can be plausibly applied to some real-world situations where single IEDs of appropriate construction are involved and await the arrival of the necessary portability and functionality of appropriate sensing technology.

\subsection{Model Validation}

In model validation, we want to prove that the virtual IEDs are representative of real-world IEDs.

We validate our system by performing the following tests;

1. Virtual Model Fidelity Test - determine the degree of exactness with which the objects in the simulation are reproduced. Note that IED model validation is already performed in our previous work [29], thus we focus our validation efforts on utilizing this method on new datasets (inert explosives and common substances).

2. Relative Density Calibration Test - evaluate the reproducibility of our relative density calibration methodology with other CT scanners. Since results obtained from scanner to scanner vary significantly (even with the same brand and model of scanner), it is important to test our calibration methodology and evaluate the consistency of the range of photometric brightness values with multiple CT scanners. Photometric brightness values should differ between CT scanners, but when following our calibration methodology (ensuring the spread of photometric brightness values (PBV) are evenly distributed and that water is calibrated to a value near or at 0.5 ), we should be able to see approximately accurate relative densities to the actual density value. 
3. Pose and Direction Accuracy Test - evaluate the accuracy of the projectile's pose and direction information obtained from the simulation - this validation was completed in [29], and receives no mention in this chapter.

For virtual model fidelity testing, we focus our validation efforts on testing the degree of 'fidelity' of the virtual model with respect to the real-world model. We compare measurements of the virtual models to real-world models and evaluate the difference between the two measurements. EDU specialist [102] suggests that an 'accurate' and acceptable virtual IED model must have similarly proportionate dimensions (length, width and height) with no more than $1 \mathrm{~cm}$ difference in magnitude, to ensure that the blast radius of the projectile from the disrupter is relatively accurate when fired at the estimated target location. We base our acceptable difference threshold from this information - and we further validate non-IED models such as inert explosives, common substances, and EDU equipment (bomb suit, EDU robot and suspicious packages).

For relative density calibration testing, we utilize 3 CT scanners with the same brand and model (Manufacturer Philips, Model: Brilliance Big Bore, Software Version: 3.6.6.17504) on the same host machine and perform the calibration process using our common substances - We evaluate the consistency of the relative PBV obtained for an inert nitro dynamite explosive with other scanners. We claim that this test is sufficient to suggest that the calibration method is a viable method to obtain relative densities of other unknown objects, using the exact same calibration method and as long as the same scanner, method, and parameters are used throughout each subsequent scan.

The validation for pose and direction accuracy test has been done in [29] in which we demonstrated that our game can support viable reasoning derived from interactions with the models inside the simulation. This process calculates the projectile pose and direction from 3D 
point coordinates taken from the instant the projectile is fired from the weapon and is displayed as output on the user's screen - and results of the projectile's pose and direction in simulation are compared to a real-world targeting system that is designed to visually indicate a projectile's start and end point.

\subsubsection{Input Datasets}

The input data sets used for our IEDs are 2 sets of DICOM images. IED datasets are previously validated in [29], and we focus our validation efforts on new datasets of inert explosives.

Dataset \#1; Substances with distinct densities (control group) consisting of honey, corn syrup, detergent, water, vegetable oil, lamp oil and rubbing alcohol - housed in $330 \mathrm{ml}$ clear water bottles.

Dataset \#2; Inert explosives consisting of semtex 10 assembly, nitro dynamite, M112 block (C-4) assembly, PETN, TNT cast booster, and smokeless black powder - housed in cardboard boxes measuring (10-12in x 5-9in x 4-6in).

Details of these explosives are described below:

Inert Semtex 10 Assembly - a general-purpose plastic explosive used in commercial blasting, demolition and military applications. Semtex was first manufactured and developed in Czechslovakia in 1964. 
Nitro dynamite - an explosive made of nitroglycerin, powdered shells or clay and stabilizers. It was patented in 1867 and widely used in mining, quarrying, construction and demolition industries.

C4 Assembly - explosives contain a highly energetic organic compound called RDX (its chemical formula is $\left.\left(\mathrm{O}_{2} \mathrm{NNCH}_{2}\right)_{3}\right)$. C4 was widely used in World War II and often used in mixtures with plasticizers or phlegmatizers.

PETN - Pentaerythritol tetranitrate $\left(\mathrm{C}_{5} \mathrm{H}_{8} \mathrm{~N}_{4} \mathrm{O}_{12}\right)$ is an explosive that when mixed with a plasticizer forms a powerful explosive.

TNT Cast Booster - this explosive booster acts as a bridge between a conventional detonator and an explosive, such as TNT. By itself, the detonator would not deliver sufficient energy to set off the charge.

Smokeless black powder - this compound consists of 55\% potassium carbonate, potassium sulfate, and potassium sulfide. It is not completely free of smoke and is used in modern semi and fully automatic firearms. 


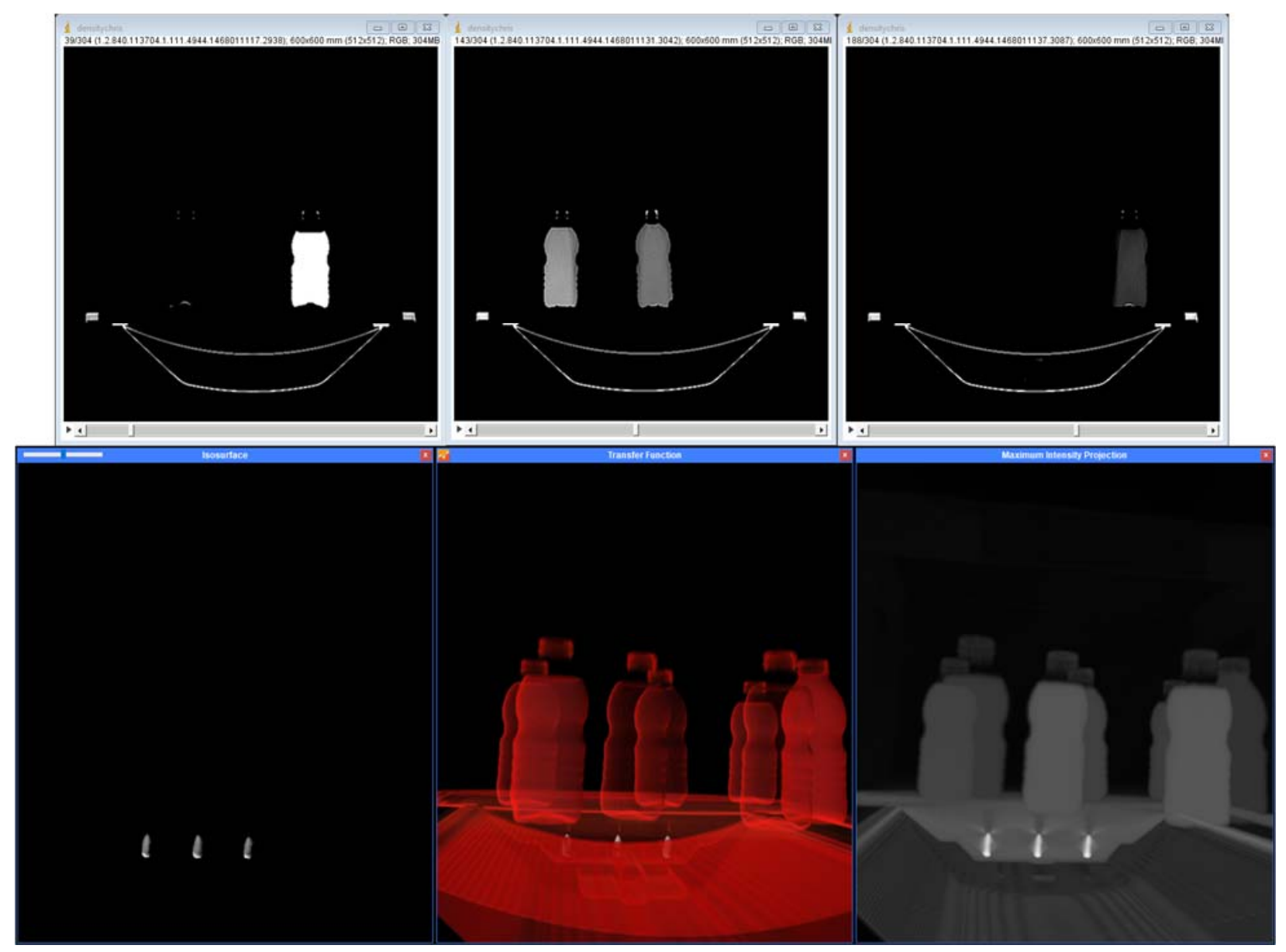

Figure 4.2 - This dataset is shown in 3 coronal views (top) and 3D reconstructed view (bottom) of substances with distinct densities. 


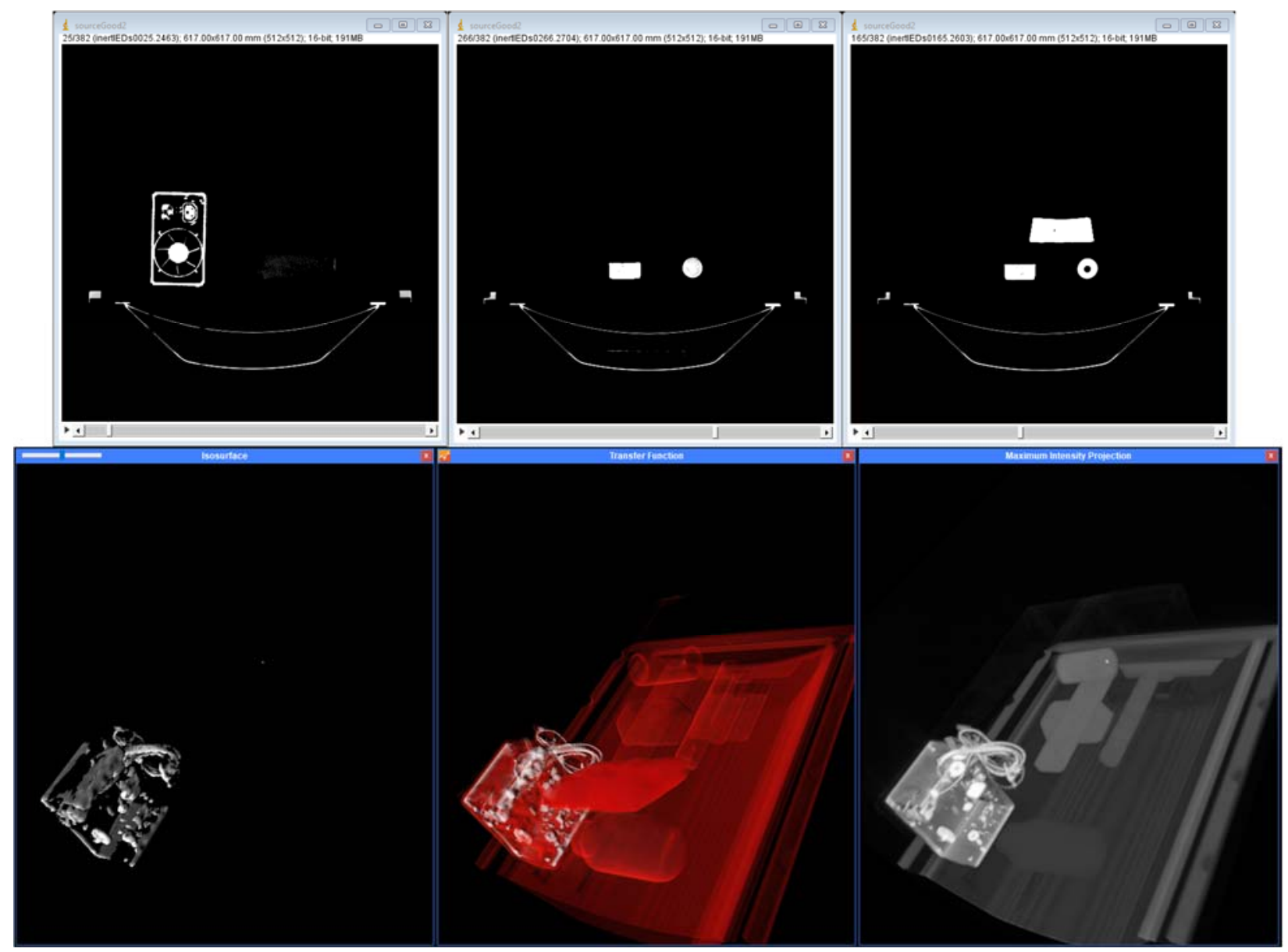

Figure 4.3 - The fourth dataset shown in 3 coronal views (top) and 3D reconstructed view (bottom) of inert explosives consisting of semtex 10 assembly, nitro dynamite, M112 block (C-4) assembly, PETN, TNT cast booster, and smokeless black powder - housed in cardboard boxes.

The dimensions of the images are 512x512. The first dataset, after final sensor calibration, contains 304 DICOM images, and the second dataset contains 382 DICOM images. Although the number of images captured can be predefined, this parameter was left automated by the CT machine which estimates how much the objects cover the surface area of the CT scanning table. These parameters were determined by a clinical medical physicist [111] as an acceptable setting for the range of materials used. 
X-rays from CT scans, however, do not use powerful magnetic fields - which allows scanning of metal implants/objects (metal nearly completely absorbs x-rays, but causes image artifacts, such as star-shaped bursts surrounding the metal object) and do not employ radio waves that may trigger an explosive initiator and cause the IED to explode.

\subsubsection{Virtual Model Fidelity Test}

The degree to which the virtual object's dimensions align to the real-world object's dimensions are provided in centimeters. We use a consistent scaling parameter to ensure that the virtual models imported into the simulation are scaled the same as all other models. Inside the simulation, we use a distance measuring tool [123] to measure game objects in the scene.

This tool allows the user to define how much one pixel equals to one unit in the real-world and automatically snaps to the vertices of game objects. The pixel-per-unit was set to $9.5714 \mathrm{px}=1$ $\mathrm{cm}$, which means a pixel measurement of 100px would equal $10.4477 \mathrm{~cm}$. We measure the number of pixels from one corner of the virtual model to another corner.

For each virtual model, we measured each side 3 times and averaged the result for each side to the ten thousandths place. The results of our measurements are presented in Table 4.1 to Table 4.3. For comparison sake, we included the measurement comparison Table 4.1 for virtual IEDs that utilized MRI scanning technology [29] so that we can evaluate the difference between the measurement comparison tables for inert IEDs using CT scanning technology. 


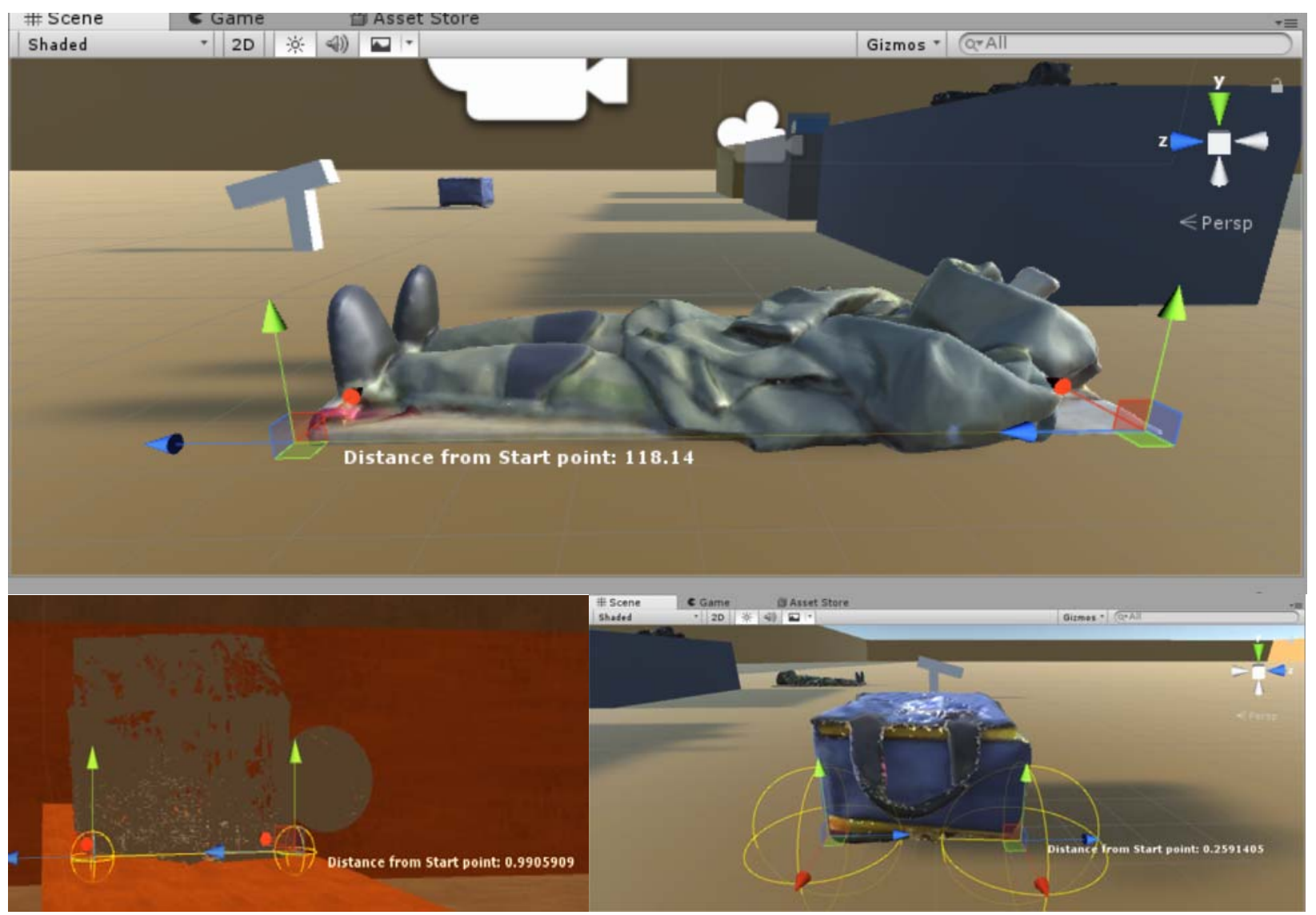

Figure 4.4-Distance tool from the Unity asset store to measure game objects in the scene.

\begin{tabular}{|c|c|c|c|c|}
\hline \multirow{2}{*}{\multicolumn{2}{|c|}{$\begin{array}{l}\text { Sim vs. Real- } \\
\text { world }\end{array}$}} & \multicolumn{2}{|c|}{ Container Dimensions } & \multirow{3}{*}{$\begin{array}{l}\text { Difference } \\
0.0397 \mathrm{~cm}\end{array}$} \\
\hline & & \multirow{2}{*}{$\begin{array}{c}\text { Model in Simulation } \\
431.0929 \mathrm{px}=45.0397 \mathrm{~cm}\end{array}$} & \multirow{2}{*}{$\frac{\text { Model in Real-world }}{45 \mathrm{~cm}}$} & \\
\hline IED & Height & & & \\
\hline Model & Width & $306.9662 \mathrm{px}=32.0712 \mathrm{~cm}$ & $32 \mathrm{~cm}$ & $0.0712 \mathrm{~cm}$ \\
\hline & Length & $143.8428 \mathrm{px}=15.0284 \mathrm{~cm}$ & $15 \mathrm{~cm}$ & $0.0284 \mathrm{~cm}$ \\
\hline \multirow{3}{*}{$\begin{array}{c}\text { IED } \\
\text { Model } \\
2\end{array}$} & Height & $192.3535 \mathrm{px}=20.0967 \mathrm{~cm}$ & $20 \mathrm{~cm}$ & $0.0967 \mathrm{~cm}$ \\
\hline & Width & $155.5352 \mathrm{px}=16.2500 \mathrm{~cm}$ & $16 \mathrm{~cm}$ & $0.2500 \mathrm{~cm}$ \\
\hline & Length & $246.6741 \mathrm{px}=25.7720 \mathrm{~cm}$ & $25 \mathrm{~cm}$ & $0.7720 \mathrm{~cm}$ \\
\hline \multirow{3}{*}{$\begin{array}{c}\text { IED } \\
\text { Model } \\
3\end{array}$} & Height & $246.6166 \mathrm{px}=25.7660 \mathrm{~cm}$ & $25 \mathrm{~cm}$ & $0.7660 \mathrm{~cm}$ \\
\hline & Width & $197.4388 \mathrm{px}=20.6280 \mathrm{~cm}$ & $20 \mathrm{~cm}$ & $0.6280 \mathrm{~cm}$ \\
\hline & Length & $141.9141 \mathrm{px}=14.8269 \mathrm{~cm}$ & $14 \mathrm{~cm}$ & $0.8269 \mathrm{~cm}$ \\
\hline & Height & $212.2668 \mathrm{px}=22.1772 \mathrm{~cm}$ & $22 \mathrm{~cm}$ & $0.1772 \mathrm{~cm}$ \\
\hline
\end{tabular}




\begin{tabular}{|c|c|c|c|c|}
\hline \multirow{2}{*}{\multicolumn{2}{|c|}{$\begin{array}{l}\text { Sim vs. Real- } \\
\text { world }\end{array}$}} & \multicolumn{2}{|c|}{ Container Dimensions } & \multirow{3}{*}{$\begin{array}{l}\text { Difference } \\
0.7157 \mathrm{~cm}\end{array}$} \\
\hline & & \multirow{2}{*}{$\begin{array}{c}\text { Model in Simulation } \\
92.9928 \mathrm{px}=9.7157 \mathrm{~cm} \\
\end{array}$} & \multirow{2}{*}{$\begin{array}{c}\text { Model in Real-world } \\
9 \mathrm{~cm} \\
\end{array}$} & \\
\hline IED & Width & & & \\
\hline 4 & Length & $222.0689 \mathrm{px}=23.2013 \mathrm{~cm}$ & $23 \mathrm{~cm}$ & $0.2013 \mathrm{~cm}$ \\
\hline \multirow{3}{*}{$\begin{array}{c}\text { IED } \\
\text { Model } \\
5\end{array}$} & Height & $224.5373 \mathrm{px}=23.4592 \mathrm{~cm}$ & $23 \mathrm{~cm}$ & $0.4592 \mathrm{~cm}$ \\
\hline & Width & $143.4705 \mathrm{px}=14.9895 \mathrm{~cm}$ & $14 \mathrm{~cm}$ & $0.9895 \mathrm{~cm}$ \\
\hline & Length & $176.6344 \mathrm{px}=18.4544 \mathrm{~cm}$ & $18 \mathrm{~cm}$ & $0.4544 \mathrm{~cm}$ \\
\hline \multirow{3}{*}{$\begin{array}{c}\text { IED } \\
\text { Model } \\
6 \\
\end{array}$} & Height & $280.5846 \mathrm{px}=29.3149 \mathrm{~cm}$ & $29 \mathrm{~cm}$ & $0.3149 \mathrm{~cm}$ \\
\hline & Width & $131.8154 \mathrm{px}=13.7718 \mathrm{~cm}$ & $13 \mathrm{~cm}$ & $0.7718 \mathrm{~cm}$ \\
\hline & Length & $194.7349 \mathrm{px}=20.3455 \mathrm{~cm}$ & $20 \mathrm{~cm}$ & $0.3455 \mathrm{~cm}$ \\
\hline
\end{tabular}

Table 4.1 - Comparison of exterior dimensions of IED containers.

\begin{tabular}{|c|c|c|c|c|}
\hline \multirow{2}{*}{\multicolumn{2}{|c|}{ Sim vs. Real-world }} & \multicolumn{2}{|c|}{ 350ml Water Bottle Dimensions } & \multirow{3}{*}{$\begin{array}{l}\text { Difference } \\
0.0239 \mathrm{~cm}\end{array}$} \\
\hline & & \multirow{2}{*}{$\begin{array}{c}\text { Model in Simulation } \\
145.7142 \mathrm{px}=15.2239 \mathrm{~cm}\end{array}$} & \multirow{2}{*}{$\begin{array}{c}\text { Model in Real-world } \\
15.2 \mathrm{~cm} \\
\end{array}$} & \\
\hline \multirow{3}{*}{$\begin{array}{c}\text { Honey } \\
\text { Model } 1\end{array}$} & Height & & & \\
\hline & Width & $64.2548 \mathrm{px}=6.7132 \mathrm{~cm}$ & $6.7 \mathrm{~cm}$ & $0.0132 \mathrm{~cm}$ \\
\hline & Length & $64.6452 \mathrm{px}=6.7540 \mathrm{~cm}$ & $6.7 \mathrm{~cm}$ & $0.0540 \mathrm{~cm}$ \\
\hline \multirow{3}{*}{$\begin{array}{c}\text { Corn } \\
\text { Syrup } \\
\text { Model } 2\end{array}$} & Height & $146.0635 \mathrm{px}=15.2604 \mathrm{~cm}$ & $15.2 \mathrm{~cm}$ & $0.0604 \mathrm{~cm}$ \\
\hline & Width & $64.7583 \mathrm{px}=6.7658 \mathrm{~cm}$ & $6.7 \mathrm{~cm}$ & $0.0658 \mathrm{~cm}$ \\
\hline & Length & $64.2886 \mathrm{px}=6.7167 \mathrm{~cm}$ & $6.7 \mathrm{~cm}$ & $0.0167 \mathrm{~cm}$ \\
\hline \multirow{3}{*}{$\begin{array}{c}\text { Detergent } \\
\text { Model } 3\end{array}$} & Height & $146.4717 \mathrm{px}=15.3031 \mathrm{~cm}$ & $15.2 \mathrm{~cm}$ & $0.1031 \mathrm{~cm}$ \\
\hline & Width & $64.6892 \mathrm{px}=6.7586 \mathrm{~cm}$ & $6.7 \mathrm{~cm}$ & $0.0586 \mathrm{~cm}$ \\
\hline & Length & $64.3925 \mathrm{px}=6.7276 \mathrm{~cm}$ & $6.7 \mathrm{~cm}$ & $0.0276 \mathrm{~cm}$ \\
\hline \multirow{3}{*}{$\begin{array}{c}\text { Water } \\
\text { Model } 4\end{array}$} & Height & $146.4389 \mathrm{px}=15.2996 \mathrm{~cm}$ & $15.2 \mathrm{~cm}$ & $0.0996 \mathrm{~cm}$ \\
\hline & Width & $64.5311 \mathrm{px}=6.7421 \mathrm{~cm}$ & $6.7 \mathrm{~cm}$ & $0.0421 \mathrm{~cm}$ \\
\hline & Length & $64.8584 \mathrm{px}=6.7763 \mathrm{~cm}$ & $6.7 \mathrm{~cm}$ & $0.0763 \mathrm{~cm}$ \\
\hline \multirow{3}{*}{$\begin{array}{c}\text { Vegetable } \\
\text { Oil } \\
\text { Model } 5\end{array}$} & Height & $146.1450 \mathrm{px}=15.2689 \mathrm{~cm}$ & $15.2 \mathrm{~cm}$ & $0.0689 \mathrm{~cm}$ \\
\hline & Width & $64.9778 \mathrm{px}=6.7887 \mathrm{~cm}$ & $6.7 \mathrm{~cm}$ & $0.0887 \mathrm{~cm}$ \\
\hline & Length & $64.3769 \mathrm{px}=6.7260 \mathrm{~cm}$ & $6.7 \mathrm{~cm}$ & $0.0260 \mathrm{~cm}$ \\
\hline \multirow{3}{*}{$\begin{array}{c}\text { Lamp Oil } \\
\text { Model } 6\end{array}$} & Height & $146.4237 \mathrm{px}=15.2980 \mathrm{~cm}$ & $15.2 \mathrm{~cm}$ & $0.0980 \mathrm{~cm}$ \\
\hline & Width & $64.4265 \mathrm{px}=6.7311 \mathrm{~cm}$ & $6.7 \mathrm{~cm}$ & $0.0311 \mathrm{~cm}$ \\
\hline & Length & $64.1508 \mathrm{px}=6.7023 \mathrm{~cm}$ & $6.7 \mathrm{~cm}$ & $0.0023 \mathrm{~cm}$ \\
\hline
\end{tabular}




\begin{tabular}{|l|c|c|c|c|}
\hline \multirow{2}{*}{ Sim vs. Real-world } & \multicolumn{2}{|c|}{ 350ml Water Bottle Dimensions } & \multirow{2}{*}{ Difference } \\
\cline { 2 - 5 } & \multicolumn{5}{|c|}{ Model in Simulation } & Model in Real-world & \\
\hline \multirow{2}{*}{$\begin{array}{l}\text { Rubbing } \\
\begin{array}{l}\text { Alcohol } \\
\text { Model 7 }\end{array}\end{array}$} & Height & $145.9057 \mathrm{px}=15.2439 \mathrm{~cm}$ & $15.2 \mathrm{~cm}$ & $0.0439 \mathrm{~cm}$ \\
\cline { 2 - 5 } & Width & $64.1584 \mathrm{px}=6.7031 \mathrm{~cm}$ & $6.7 \mathrm{~cm}$ & $0.0031 \mathrm{~cm}$ \\
\cline { 2 - 5 } & Length & $64.6283 \mathrm{px}=6.7522 \mathrm{~cm}$ & $6.7 \mathrm{~cm}$ & $0.0522 \mathrm{~cm}$ \\
\hline
\end{tabular}

Table 4.2 - Comparison of exterior dimensions of $350 \mathrm{ml}$ water bottle containers with different substances.

\begin{tabular}{|c|c|c|c|c|}
\hline \multirow{2}{*}{\multicolumn{2}{|c|}{ Sim vs. Real-world }} & \multicolumn{2}{|c|}{ Inert Explosive Dimensions } & \multirow{3}{*}{$\begin{array}{l}\text { Difference } \\
0.0994 \mathrm{~cm}\end{array}$} \\
\hline & & \multirow{2}{*}{$\begin{array}{c}\text { Model in Simulation } \\
44.0229 \mathrm{px}=4.5994 \mathrm{~cm}\end{array}$} & \multirow{2}{*}{$\begin{array}{c}\text { Model in Real-world } \\
4.5 \mathrm{~cm}\end{array}$} & \\
\hline \multirow{3}{*}{$\begin{array}{l}\text { Semtex 10 } \\
\text { Assembly }\end{array}$} & Height & & & \\
\hline & Width & $29.3719 \mathrm{px}=3.0687 \mathrm{~cm}$ & $3 \mathrm{~cm}$ & $0.0687 \mathrm{~cm}$ \\
\hline & Length & $72.4512 \mathrm{px}=7.5696 \mathrm{~cm}$ & $7.5 \mathrm{~cm}$ & $0.0696 \mathrm{~cm}$ \\
\hline \multirow{3}{*}{$\begin{array}{c}\text { Nitro } \\
\text { Dynamite }\end{array}$} & Height & $24.3385 \mathrm{px}=2.5428 \mathrm{~cm}$ & $2.5 \mathrm{~cm}$ & $0.0428 \mathrm{~cm}$ \\
\hline & Width & $23.9501 \mathrm{px}=2.5023 \mathrm{~cm}$ & $2.5 \mathrm{~cm}$ & $0.0023 \mathrm{~cm}$ \\
\hline & Length & $306.8190 \mathrm{px}=32.0558 \mathrm{~cm}$ & $32 \mathrm{~cm}$ & $0.0558 \mathrm{~cm}$ \\
\hline \multirow{3}{*}{$\begin{array}{c}\text { M112 } \\
\text { Block } \\
\text { (C4) } \\
\text { Assembly } \\
\end{array}$} & Height & $29.3164 \mathrm{px}=3.0629 \mathrm{~cm}$ & $3 \mathrm{~cm}$ & $0.0629 \mathrm{~cm}$ \\
\hline & Width & $43.1991 \mathrm{px}=4.5134 \mathrm{~cm}$ & $4.5 \mathrm{~cm}$ & $0.0134 \mathrm{~cm}$ \\
\hline & Length & $134.6071 \mathrm{px}=14.0635 \mathrm{~cm}$ & $14 \mathrm{~cm}$ & $0.0635 \mathrm{~cm}$ \\
\hline \multirow{3}{*}{ PETN } & Height & $34.4823 \mathrm{px}=3.6026 \mathrm{~cm}$ & $3.5 \mathrm{~cm}$ & $0.1026 \mathrm{~cm}$ \\
\hline & Width & $57.6448 \mathrm{px}=6.0226 \mathrm{~cm}$ & $6 \mathrm{~cm}$ & $0.0226 \mathrm{~cm}$ \\
\hline & Length & $129.9293 \mathrm{px}=13.5747 \mathrm{~cm}$ & $13.5 \mathrm{~cm}$ & $0.0747 \mathrm{~cm}$ \\
\hline \multirow{3}{*}{$\begin{array}{l}\text { TNT Cast } \\
\text { Booster }\end{array}$} & Height & $29.2857 \mathrm{px}=3.0597 \mathrm{~cm}$ & $3 \mathrm{~cm}$ & $0.0597 \mathrm{~cm}$ \\
\hline & Width & $29.0603 \mathrm{px}=3.0362 \mathrm{~cm}$ & $3 \mathrm{~cm}$ & $0.0362 \mathrm{~cm}$ \\
\hline & Length & $96.6516 \mathrm{px}=10.0980 \mathrm{~cm}$ & $10 \mathrm{~cm}$ & $0.0980 \mathrm{~cm}$ \\
\hline \multirow{3}{*}{$\begin{array}{c}\text { Smokeless } \\
\text { Black } \\
\text { Powder }\end{array}$} & Height & $182.4469 \mathrm{px}=19.0617 \mathrm{~cm}$ & $19 \mathrm{~cm}$ & $0.0617 \mathrm{~cm}$ \\
\hline & Width & $53.4496 \mathrm{px}=5.5843 \mathrm{~cm}$ & $5.5 \mathrm{~cm}$ & $0.0843 \mathrm{~cm}$ \\
\hline & Length & $53.3563 \mathrm{px}=5.5746 \mathrm{~cm}$ & $5.5 \mathrm{~cm}$ & $0.0746 \mathrm{~cm}$ \\
\hline
\end{tabular}

Table 4.3 - Comparison of exterior dimensions of inert explosives. 
We notice that the dimensions of the models in the simulation are consistently larger than the dimensions of the models in reality, with an average difference of $0.4393 \mathrm{~cm}$ for the inert IEDs, $0.0536 \mathrm{~cm}$ for water bottle containers, and $0.0607 \mathrm{~cm}$ for the inert explosives.

In our previous study, we employed the use of a Magnetic Resonance Image scanner that tends to capture more artifacts (streaks and lines) - which may suggest the increase in average difference $0.4393 \mathrm{~cm}$ compared to that of our current study which uses a CT scanner that yielded results of $0.0536 \mathrm{~cm}$ and $0.0607 \mathrm{~cm}$ in differences. The reason for this may be that metal objects in the MRI scanner tend to cause more distortion in the images after rendering due to the finite signal mapping of magnetic pulses. However, using the distance tool to measure the farthest pixel of each virtual model would generally result in a larger measurement.

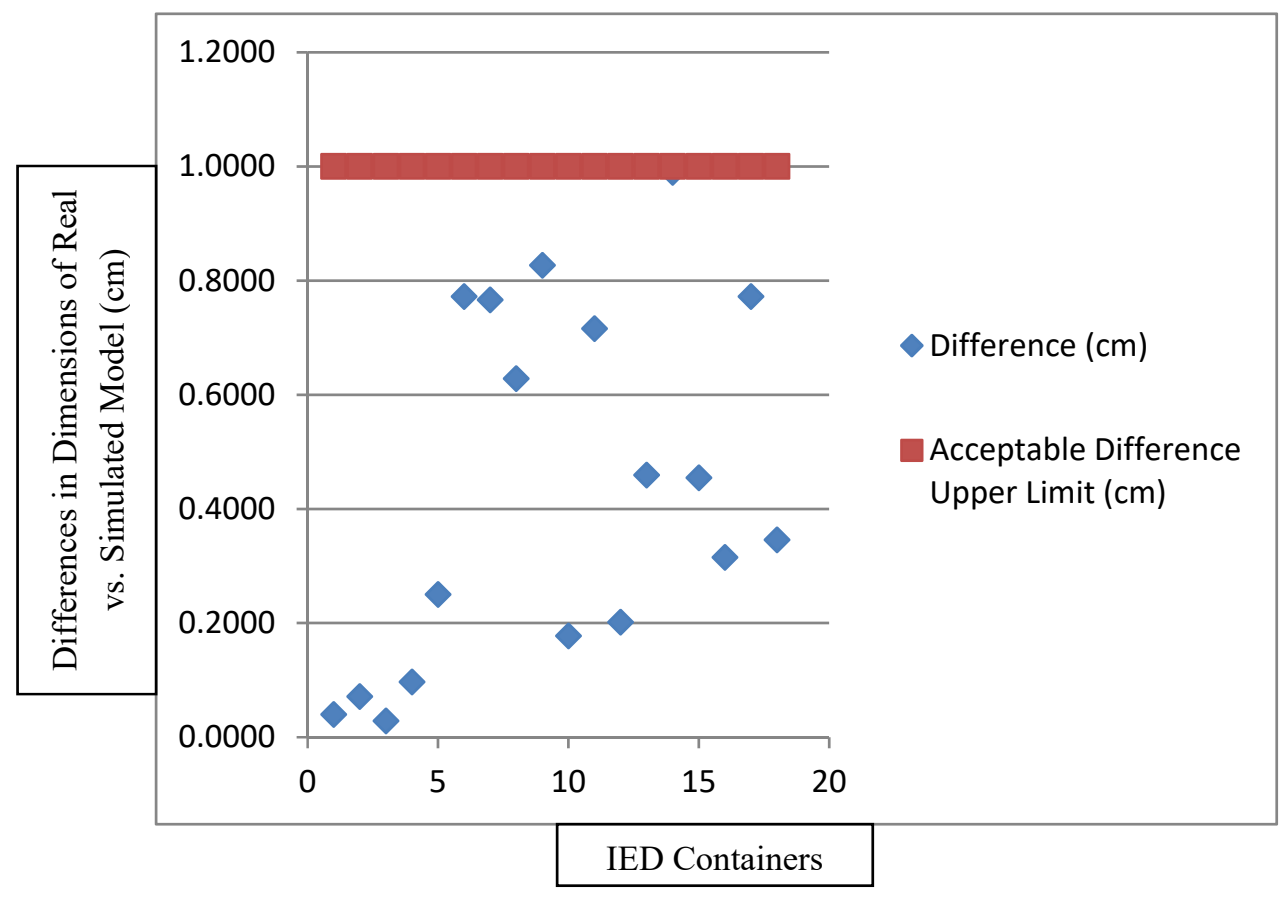

Figure 4.5 - Differences in dimensions of the exterior of IED containers, upper limit determined by EDU specialists [102].

We study the dimensions of the virtual models and compare them to its respective realworld dimensions because we want to determine the accuracy of our simulation. The degree of 
which we can accurately model real-world objects and provide relevant information to EDU professionals are important parts of an accurate simulation as it is expected to produce accurate physical interactions with its virtual models.

We decided that by measuring and comparing the dimensions of these virtual models and showing that the differences in dimensions that are within the acceptable $1 \mathrm{~cm}$ range, we can prove 3 elements that affect EDU neutralization processes; these elements are; 1. in-game physical interactions that are calculated with these virtual models are possible, 2. differences in form, size, shape and even density do not affect the outcome of the virtual model, and 3. occluded virtual models are also accurate. With accurate dimensions, we can prove these 3 elements are true and the simulation is capable of providing accurate $3 \mathrm{D}$ real-world physical interaction representations.

EDU professionals testified that the acceptable difference between a real-world model and a simulated model should be no more than $1 \mathrm{~cm}$. The reason behind this threshold is that the use of a neutralization cannon will have a wide enough blast radius that will negate results that have differences in measurements by $1 \mathrm{~cm}$.

Figure 4.5 effectively shows that the difference of simulated models and the real-world models are well below the $1 \mathrm{~cm}$ acceptable difference, it emphasizes that the simulation is capable of producing relatively accurate simulated models captured from real-world models. These substances are chosen because they are common household products and cover an ample range of high and low densities. 


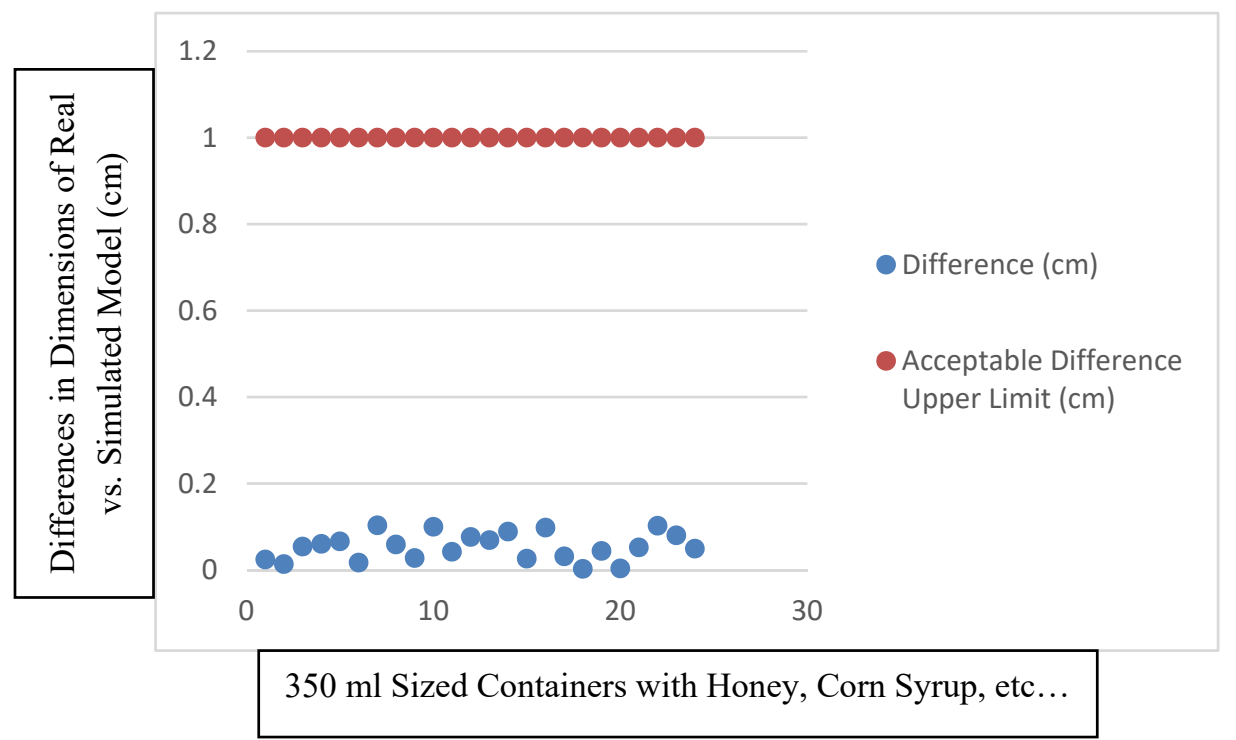

Figure 4.6 - Differences in dimensions of exterior dimensions of $350 \mathrm{ml}$ water bottle containers with honey, corn syrup, detergent, water, vegetable oil, and lamp oil. Upper limit determined by EDU specialists [102].

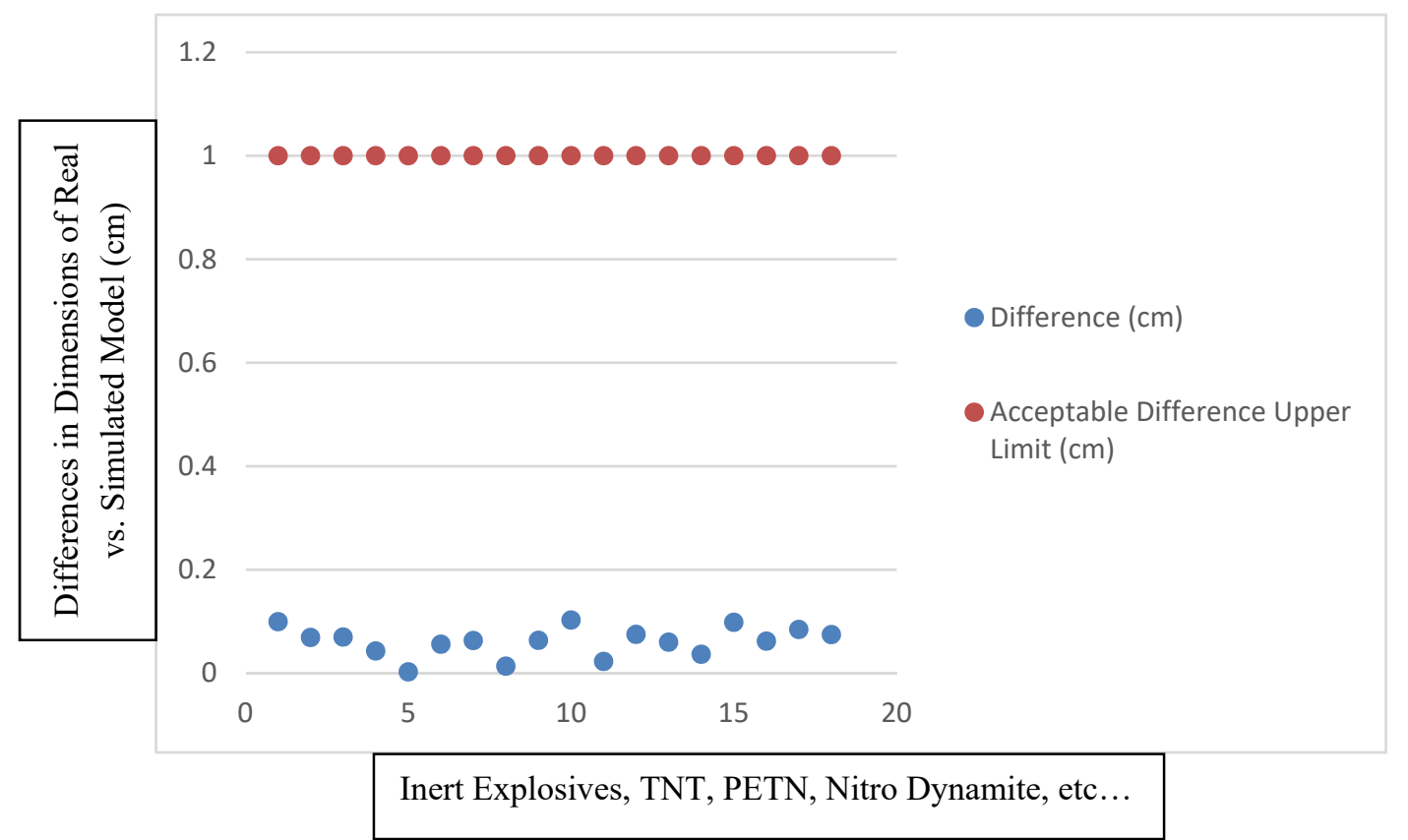

Figure 4.7 - Differences in dimensions of exterior dimensions of inert explosives. Upper limit determined by EDU specialists [102]. 
Figure 4.6 and figure 4.7 shows that the difference of simulated models and the real-world models of varying substances and inert explosives are still well below the $1 \mathrm{~cm}$ acceptable difference, it emphasizes that the simulation is capable of producing relatively accurate simulated models captured from real-world models despite differences in size, shape, form and density. These inert explosives are chosen because they are commonly seen and used in EDU training exercises.

Figure 4.6 and figure 4.7 show that the differences in magnitude are well within the EDUdefined acceptable virtual IED model limit of no more than $1 \mathrm{~cm}$. Although this result is within the range of an 'accurate' virtual IED model by an EDU specialist, it is sufficient to provide weight of evidence that relatively accurate models can be achieved with these exact CT scanning parameters, surrounding conditions and simulation scaling. However, this result does provide weight of evidence that virtual IEDs, despite being comprised of different materials, can still have similarly proportionate dimensions (length, width and height) with no more than $1 \mathrm{~cm}$ difference in magnitude. Which means that the method of CT scanning is feasible to capture different compositions of IEDs without comprising its dimensional proportions due to discrepancies during finite sampling.

We perform another analysis on the dimensions of each IED components and observe whether differences exist when capturing data from occluded objects. This process is important to evaluate because scanning occluded objects are core to the contributions of this thesis. Scanned occluded models need to be accurate so that calculations performed in game can be relevant and as close as possible to real-world interactions for EDU to make a reasoned decision. 


\begin{tabular}{|c|c|c|c|c|c|}
\hline $\begin{array}{l}\text { Sim vs. } \\
\text { Reality }\end{array}$ & Components & Type of Material & $\begin{array}{c}\text { Virtual Components' } \\
\text { Dimensions (LxWxH) } \\
\text { in } \mathrm{cm}\end{array}$ & $\begin{array}{c}\text { Real-world } \\
\text { Components' } \\
\text { Dimensions (LxWxH) } \\
\text { in cm }\end{array}$ & $\begin{array}{l}\text { Average Difference } \\
(\mathrm{cm})\end{array}$ \\
\hline $\begin{array}{c}\text { Model } \\
1 \\
\end{array}$ & $\begin{array}{l}\text { Cellular Device } \\
\text { Charger }\end{array}$ & $\begin{array}{l}\text { Metal/Plastic } \\
\text { Metal/Plastic }\end{array}$ & $\begin{array}{c}10.6 \times 4.2 \times 1.8 \\
6.5 \times 4.6 \times 2.7 \\
\end{array}$ & $\begin{array}{c}10.0 \times 4.0 \times 1.0 \\
6.0 \times 4.0 \times 2.0\end{array}$ & $\begin{array}{l}0.5326 \\
0.5999\end{array}$ \\
\hline $\begin{array}{l}\text { Model } \\
2\end{array}$ & $\begin{array}{l}\text { Cellular Device } \\
\text { Power Source } \\
\text { Explosive }\end{array}$ & $\begin{array}{l}\text { Metal/Plastic } \\
\text { Metal/Plastic } \\
\text { Wood }\end{array}$ & $\begin{array}{c}11.5 \times 5.3 \times 2.6 \\
5.0 \times 3.3 \times 1.7 \\
15.9 \times 6.3 \times 6.0\end{array}$ & $\begin{array}{c}11.0 \times 4.5 \times 2.0 \\
4.5 \times 2.5 \times 1.5 \\
15.0 \times 6.0 \times 6.0\end{array}$ & $\begin{array}{l}0.6547 \\
0.4970 \\
0.4013\end{array}$ \\
\hline $\begin{array}{l}\text { Model } \\
3\end{array}$ & $\begin{array}{l}\text { Cellular Device } \\
\text { Power Source } \\
\text { Explosive }\end{array}$ & $\begin{array}{l}\text { Metal/Plastic } \\
\text { Metal/Plastic } \\
\text { Wood } \\
\text { Glass/Liquid }\end{array}$ & $\begin{array}{c}8.7 \times 5.9 \times 1.5 \\
4.5 \times 2.9 \times 1.6 \\
20.2 \times 5.8 \times 5.7 \\
14.8 \times 7.8 \times 12.2\end{array}$ & $\begin{array}{c}8.5 \times 5.0 \times 1.5 \\
4.5 \times 2.5 \times 1.5 \\
20.0 \times 5.5 \times 5.5 \\
14.0 \times 7.0 \times 12.0\end{array}$ & $\begin{array}{l}0.3654 \\
0.1752 \\
0.2590 \\
0.5678\end{array}$ \\
\hline $\begin{array}{l}\text { Model } \\
4\end{array}$ & $\begin{array}{l}\text { Cellular Device } \\
\text { Power Source } \\
\text { Explosive }\end{array}$ & $\begin{array}{l}\text { Metal/Plastic } \\
\text { Metal/Plastic } \\
\text { Wood } \\
\text { Plastic/Liquid }\end{array}$ & $\begin{array}{c}11.0 \times 5.3 \times 1.2 \\
4.5 \times 2.6 \times 1.6 \\
20.9 \times 6.5 \times 6.9 \\
15.3 \times 6.5 \times 6.2\end{array}$ & $\begin{array}{c}10.5 \times 4.5 \times 1.0 \\
4.5 \times 2.5 \times 1.5 \\
20.0 \times 6.0 \times 6.0 \\
15.0 \times 6.0 \times 6.0\end{array}$ & $\begin{array}{l}0.4841 \\
0.0518 \\
0.7685 \\
0.3539\end{array}$ \\
\hline $\begin{array}{l}\text { Model } \\
5\end{array}$ & $\begin{array}{l}\text { Cellular Device } \\
\text { Power Source } \\
\text { Explosive }\end{array}$ & $\begin{array}{l}\text { Metal/Plastic } \\
\text { Metal/Plastic } \\
\text { Metal }\end{array}$ & $\begin{array}{c}10.1 \times 5.0 \times 1.7 \\
4.8 \times 2.6 \times 1.8 \\
16.0 \times 7.2 \times 3.8\end{array}$ & $\begin{array}{c}10.0 \times 4.0 \times 1.5 \\
4.5 \times 2.5 \times 1.5 \\
15.0 \times 7.0 \times 3.5\end{array}$ & $\begin{array}{l}0.4301 \\
0.2203 \\
0.4946\end{array}$ \\
\hline $\begin{array}{l}\text { Model } \\
6\end{array}$ & $\begin{array}{l}\text { Cellular Device } \\
\text { Power Source } \\
\text { Explosive }\end{array}$ & $\begin{array}{l}\text { Metal/Plastic } \\
\text { Metal/Plastic } \\
\text { Wood } \\
\text { Plastic/Wood } \\
\text { Metal }\end{array}$ & $\begin{array}{c}7.5 \times 5.2 \times 3.5 \\
5.1 \times 3.3 \times 2.0 \\
20.3 \times 6.0 \times 6.3 \\
15.8 \times 4.2 \times 4.2 \\
20.0 \times 8.4 \times 8.3\end{array}$ & $\begin{array}{c}7.0 \times 5.0 \times 2.5 \\
4.5 \times 2.5 \times 1.5 \\
19.5 \times 6.0 \times 6.0 \\
15.0 \times 3.5 \times 3.5 \\
19.0 \times 7.5 \times 7.5\end{array}$ & $\begin{array}{l}0.5529 \\
0.6249 \\
0.3967 \\
0.7098 \\
0.8893\end{array}$ \\
\hline
\end{tabular}

Table 4.4 - Comparison of dimensions of IED components.

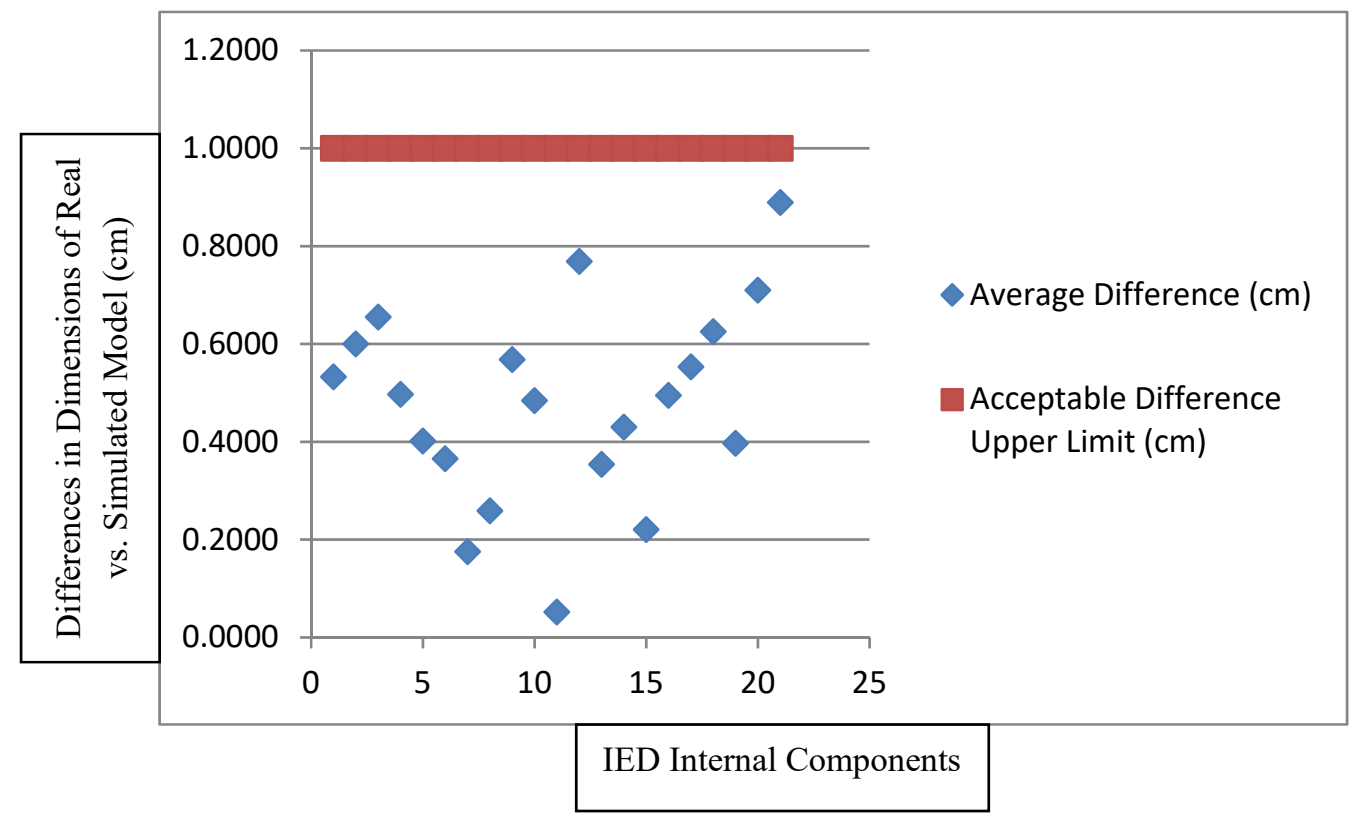

Figure 4.8 - Differences in dimensions of IED components. 
Since having accurate IED components are a crucial element in aiding EDU to determine points of interests and possible points of neutralization, we focus on determining whether scanning occluded objects within a container significantly affects the accuracy of its dimensions. Many of the contributions of this thesis rely on reasoning directly from the simulation's virtual model's and its interior components, therefore the IED components are evaluated for its accuracy.

The average difference in dimensions of IED components is $0.4383 \mathrm{~cm}$, and this result shows no noticeable change in the quality of the scan due to the components being hidden inside the container. There is also no significant increase or decrease when compared to the difference in dimensions of the exterior, implying that occluded objects can be captured just as well as objects that are not occluded. Measurements imply that CT scans are still relatively 'accurate' (within 1 cm difference in magnitude) even when scanning objects that are occluded and gives support to the assertion that $\mathrm{CT}$ scanning can possibly be adapted to support EDU processes which rely heavily on identifying occluded objects.

However, it is important to note that the system relies on human interaction to visually remove major artifacts such as streaks and overflow marks. It is possible that the uncanny nature of IEDs may cause the important protrusions or elements to be misinterpreted as an artifact, thus resulting in misrepresented real-world IED based on an erroneous assumption that the sensing method introduced artificial artifacts. This problem is beyond the scope of this thesis but may lead to serious consequences. 


\subsubsection{Relative Density Calibration Test}

In this section, we evaluate the reproducibility of our relative density calibration methodology using other CT scanners. In our experiments, we had access to $3 \mathrm{CT}$ scanners. For each scanner we applied our relative density calibration methodology:

1. Scan the range of common substances with variance of density values using the head and neck protocol (see section 3.2.2 for specific parameters of this protocol) - for this part we selected the following common substances: honey, corn syrup, detergent, water, vegetable oil, lamp oil and rubbing alcohol.

2. Adjust the technical parameters mentioned in section 3.2.2 (which are tube current-time product, slice thickness, resolution, field of measurement) and rescan common substances until the range of photometric brightness values (PBV) are distributed from 0 to 1 , with water at a value near or at 0.5 , plus or minus a difference of $0.1(0.5$ is a heuristic value reasoned from the fact that water is our selected baseline)

3. Only until step 2 is satisfied and the sensor is calibrated sufficiently, can we evaluate the consistency of the relative PBV obtained for an inert nitro dynamite explosive (an inert explosive designed with chemical properties analogous to its explosive counterpart).

If the inert nitro dynamite explosive outputs a PBV that suggests that it is within the range of this explosive, then we claim that this test is adequate to suggest that the calibration method is a viable method to obtain relative densities of other unknown objects as long as the same scanner, method, and parameters are used throughout each subsequent scan.

We show the resulting PBV measurements in Table 4.5 to 4.8 . 
CT Scanner 1 - Photometric Brightness Values for Common Substances

\begin{tabular}{|c|c|c|c|c|}
\hline Substance & $\begin{array}{l}\text { Density } \\
\left(\mathrm{g} / \mathrm{cm}^{3}\right)\end{array}$ & Initial Scan & $\begin{array}{c}\text { After } 1^{\text {st }} \\
\text { Calibration }\end{array}$ & $\begin{array}{c}\text { After } 2^{\text {nd }} \\
\text { Calibration }\end{array}$ \\
\hline Honey & 1.42 & 0.9998 & 0.9581 & 0.9129 \\
\hline Corn Syrup & 1.33 & 0.9993 & 0.9536 & 0.9196 \\
\hline Detergent & 1.06 & 0.8519 & 0.8195 & 0.7419 \\
\hline Water & 1.00 & 0.6298 & 0.5809 & 0.5398 \\
\hline Vegetable Oil & 0.92 & 0.4214 & 0.3892 & 0.2903 \\
\hline Lamp Oil & 0.81 & 0.3239 & 0.3090 & 0.2670 \\
\hline Rubbing Alcohol & 0.79 & 0.0062 & 0.0092 & 0.0494 \\
\hline
\end{tabular}

Table 4.5 - Photometric brightness values obtained using CT scanner 1 for common substances shown in 3 different scans with slightly modified parameters.

\section{CT Scanner 2 - Photometric Brightness Values for Common Substances}

\begin{tabular}{|c|c|c|c|c|}
\hline Substance & Density $\left.\mathbf{( g / \mathbf { c m } ^ { \mathbf { 3 } }}\right)$ & Initial Scan & After $\mathbf{1}^{\text {st }}$ Calibration & After $2^{\text {nd }}$ Calibration \\
\hline Honey & 1.42 & 0.9999 & 0.9756 & 0.9199 \\
\hline Corn Syrup & 1.33 & 0.9994 & 0.9448 & 0.8906 \\
\hline Detergent & 1.06 & 0.8527 & 0.8417 & 0.7669 \\
\hline Water & 1.00 & 0.6360 & 0.6032 & 0.5309 \\
\hline Vegetable Oil & 0.92 & 0.4304 & 0.4209 & 0.3533 \\
\hline Lamp Oil & 0.81 & 0.3314 & 0.3137 & 0.2581 \\
\hline Rubbing Alcohol & 0.79 & 0.0092 & 0.0399 & 0.0131 \\
\hline
\end{tabular}

Table 4.6 - Photometric brightness values obtained using CT scanner 2 for common substances shown in 3 different scans with slightly modified parameters.

\section{CT Scanner 3 - Photometric Brightness Values for Common Substances}

\begin{tabular}{|c|c|c|c|c|}
\hline Substance & Density $\mathbf{( g / \mathbf { c m } ^ { \mathbf { 3 } } )}$ & Initial Scan & After $\mathbf{1}^{\text {st }}$ Calibration & After 2 $^{\text {nd }}$ Calibration \\
\hline Honey & 1.42 & 0.9999 & 0.9978 & 0.9649 \\
\hline Corn Syrup & 1.33 & 0.9997 & 0.9723 & 0.8953 \\
\hline Detergent & 1.06 & 0.8590 & 0.8079 & 0.7603 \\
\hline Water & 1.00 & 0.6324 & 0.5853 & 0.5430 \\
\hline Vegetable Oil & 0.92 & 0.4247 & 0.3784 & 0.3125 \\
\hline Lamp Oil & 0.81 & 0.3298 & 0.2969 & 0.2470 \\
\hline $\begin{array}{c}\text { Rubbing } \\
\text { Alcohol }\end{array}$ & 0.79 & 0.0083 & 0.0478 & 0.0226 \\
\hline
\end{tabular}

Table 4.7 - Photometric brightness values obtained using CT scanner 3 for common substances shown in 3 different scans with slightly modified parameters. 
After following the procedure for developing a lookup table to analyze unknown substances shown in section 3.2.3 and 3.2.4, we scanned an inert nitro dynamite with each calibrated CT scanner to see if the results are consistent. The relative PBV results of the inert nitro dynamite are shown in Table 4.8:

\begin{tabular}{|c|c|c|}
\hline Nitro Dynamite Inert Explosive & Density $\left(\mathrm{g} / \mathrm{cm}^{3}\right)$ & Value (in HSV colour space) \\
\hline CT Scanner 1 & 1.6001 & 0.9993 \\
\hline CT Scanner 2 & 1.6667 & 0.9994 \\
\hline CT Scanner 3 & 1.6680 & 0.9995 \\
\hline
\end{tabular}

Table 4.8 - This table shows the validation results of scanning an inert nitro dynamite on 3 different CT scanners, the PBV obtained from each scanner is shown in the rightmost column, and the density that each PBV is associated with is shown in the middle column.

Table 4.8 shows that the scanned PBV for inert nitro dynamite correlates with a density value that is at $1.6 \mathrm{~g} / \mathrm{cm}^{3}$. The real-world density value of nitro dynamite is $1.6 \mathrm{~g} / \mathrm{cm}^{3}$, according to DSA detection [105] and this suggests that our relative density measurements are accurate for scanning inert nitro dynamite and we claim that it is also accurate for evaluating unknown objects to a high degree of certainty for its density value.

We argue that these validation results support the claim of our objectives; namely; providing a physically accurate representation of an IED that maintains all spatial relationships between its components and providing information regarding a range of the type of explosive(s) employed in the device and ascertain to a degree of certainty the nature of the unknown materials in a concealed container.

We also claim that our validation suggests the possibility of scanning and creating 3D virtual models of any EDU object (not only IED models as suggested in our previous work [29]), given that the target object is size appropriate for placement in the scanner. 
We also suggest that employing our structured scanning procedure can theoretically be applied to any scanner, and may not require an expert technician to calibrate sensor parameters). 


\subsection{Splatter Impact Point Validation}

In this section, we demonstrate that our game-based simulation is capable of predicting accurate splatter impact points as a result of an effective IED neutralization shot.

We performed two test methods to validate our splatter impact model in our simulation.

- Test method 1 consists of testing the dispersion of a single projectile onto a tight liquid mass - essentially one paintball pellet fired from a paintball gun into a water dyed water balloon. We analyzed the dispersion of the liquid by using a sensor grid pad, evaluated the percentage of the affected area of each grid, and evaluated the accuracy of our simulated model grid output to the real-world grid output.

- Test method 2 consist of testing the dispersion of multiple projectiles onto multiple masses of varying densities - essentially multiple water projectiles from a disrupter cannon into a target consisting of multiple components. We also analyzed the dispersion of the liquid by using a sensor grid pad, evaluated the percentage of the affected area of each grid, and evaluated the accuracy of our simulated model grid output to the real-world grid output.

\subsubsection{Single Projectile to One Mass}

To complete this evaluation, we validated our simulation results with real-life experiments of a high velocity water projectile impacting on containers of liquid. The goal of this experiment is to test the base case of the simulation being able to model accurately to a degree of certainty a single projectile hitting one mass, if this base case is successful, then we may confidently proceed 
to model multiple projectiles (a disruptor shot) to multiple masses (multiple objects). For our initial experiments, we demonstrate our results with the following test equipment shown in Table 4.9.

\begin{tabular}{|c|c|c|}
\hline Form & Purpose & Characteristics \\
\hline $\begin{array}{l}\text { Automatic feed marking pellet gun } \\
\text { (TIPPMAN A-5 Paintball Marker) }\end{array}$ & Weapon & $\begin{array}{l}\text { High Pressure Air aka. compressed air with (180-850 } \\
\text { controlled constant psi, consistent velocity) } \\
\text { Adjustable muzzle velocity }\end{array}$ \\
\hline 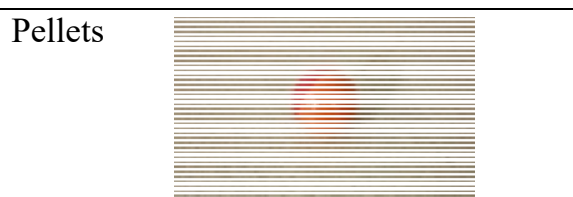 & $\begin{array}{l}\text { Projectile for } \\
\text { Weapon }\end{array}$ & $\begin{array}{l}\text { Coloured } \\
0.68 \text { caliber }\end{array}$ \\
\hline 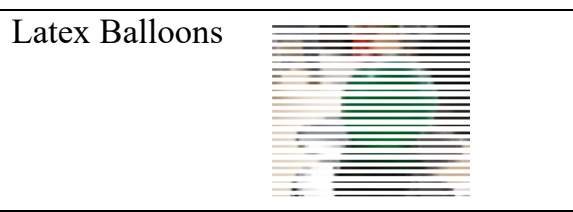 & Target & $\begin{array}{l}3 \mathrm{~cm} \text { radius, filled with tap water } \\
0.0063 \text { ounce / piece } \\
\text { Natural latex EN71 Certification }\end{array}$ \\
\hline Weapon Tripod & $\begin{array}{l}\text { Control } \\
\text { Harness }\end{array}$ & Height adjustable stand for the weapon \\
\hline Bubble level & $\begin{array}{l}\text { Horizontal } \\
\text { Plane Setup }\end{array}$ & Ensure gun and target are at level height \\
\hline $\begin{array}{l}\text { Pellet Chronographer (Radarchron - } \\
\text { Doppler radar velocity sensor) }\end{array}$ & $\begin{array}{l}\text { Control } \\
\text { Speed }\end{array}$ & Measure the speed of a projectile \\
\hline $\begin{array}{l}\text { High Definition Video Camera (GoPro } \\
\text { Hero 6) }\end{array}$ & $\begin{array}{l}\text { Control } \\
\text { Impact Point }\end{array}$ & $\begin{array}{l}\text { Various GoPro Hero } 6 \text { shooting a 1080p video @ } 240 \\
\text { frames per second at different angles. }\end{array}$ \\
\hline
\end{tabular}

Table 4.9 - Our test equipment shown here includes a paintball gun, a pellet chronographer and HD video cameras. 
We measured the dispersion splatter with a 5 by 5 sensor grid pad with each pad measuring 8 1/2 x 11 inches. Splatter impact is visually estimated by the percentage of disrupted surface area.

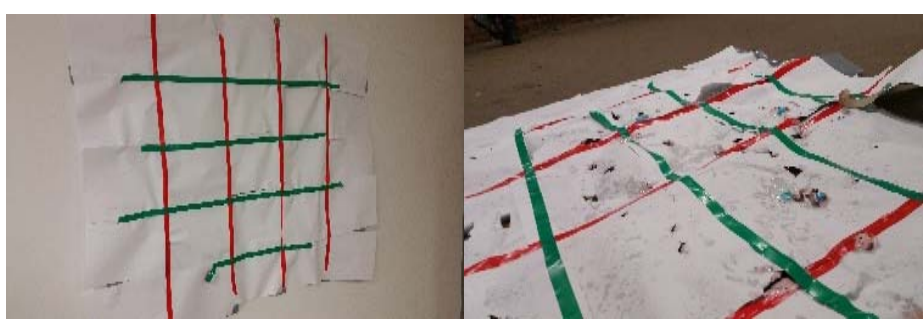

Figure 4.9 - Sensor grid pad labeled starting from top left as grid A1 to E5 bottom right.

Our test method is as follows:

1. Setup weapon apparatus, HD camera, sensor grid and target water balloon (filled with food coloured tap water to a spherical shape of approximately $3 \mathrm{~cm}$ radius) at $2 \mathrm{~m}$ from the target water balloon to the weapon apparatus, and the sensor grid at $20 \mathrm{~cm}$ behind the target water balloon.

2. Load paintball gun with coloured 0.68 caliber pellets.

3. Measure the speed of the pellet from the paintball gun with chronographer.

4. Calibrate the speed of the paintball pellet shot (approximately the rated speed of 89 $\mathrm{m} / \mathrm{s}$ ) and ensure subsequent shots are consistent with this speed.

5. Aim paintball gun at the centre of the water balloon, and verify with some trial shots.

6. Shoot one paintball pellet, and record the shot in 240 frames per second.

7. Visually inspect the splatter impact points (splatter from the dispersion of the water balloon after the pellet has hit the target) and record the percentage of each grid that is affected by the splatter. Average results.

8. Repeat step 6-7 for 10 times, replacing the sensor grid each time. 
Since the projectile's initial point of contact with the target surface significantly modifies the dispersion of the particles, we ensure consistent impact points by freeze framing the impact from of a 240 frames per second camera attached on the weapon's aim and visually adjusting our simulation's point of impact to match the target impact point.

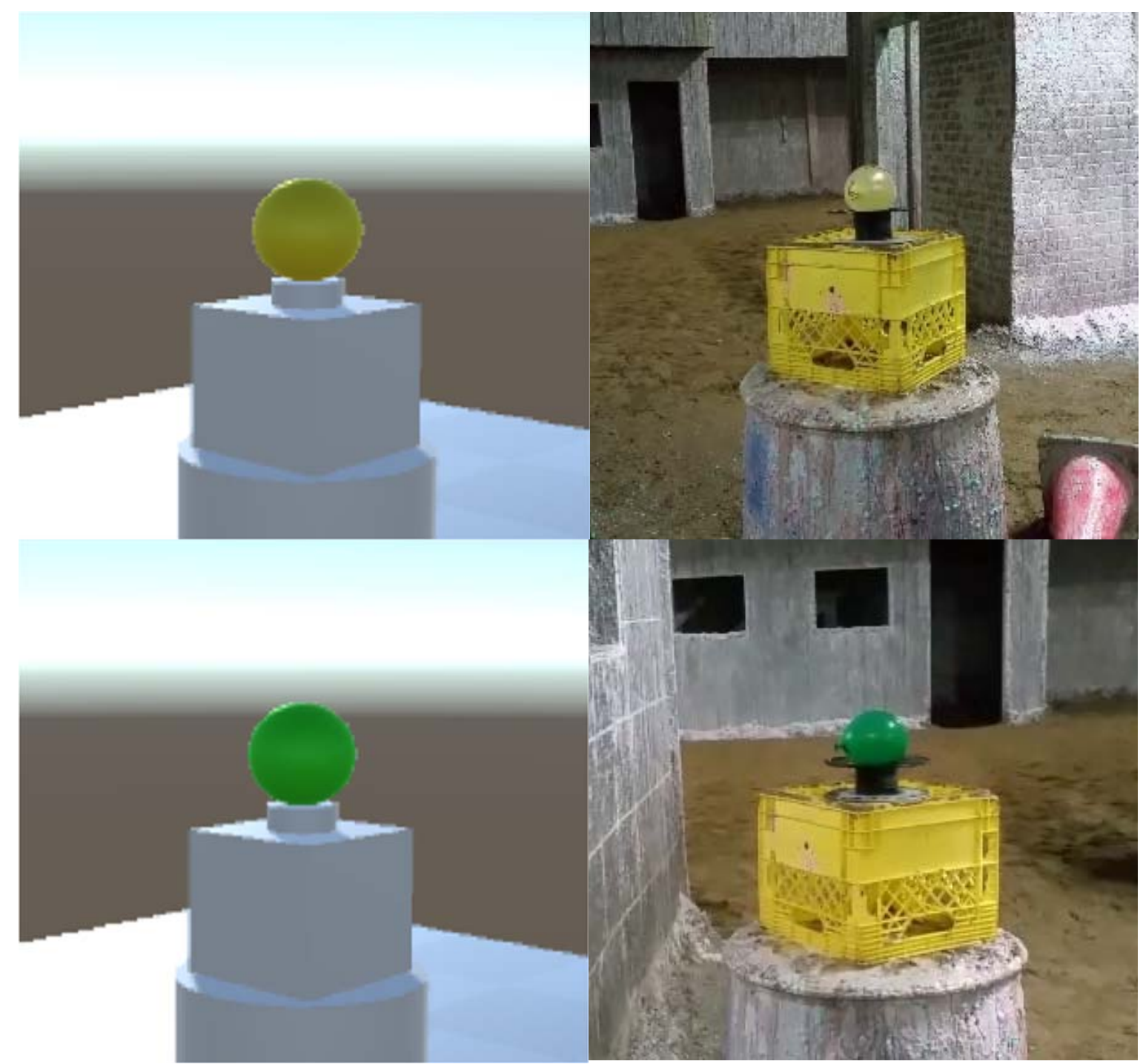

Figure 4.10 - Left: The target object as a customized Unity particle model. Right: Real-world target object. 
The simulation also is provided with a digital surface grid that is configured to match the real-world sensor grid pad. Depending on the forces applied to the particles, the simulation computes the particle's destination and outputs onto console the percentages that each grid has been affected.

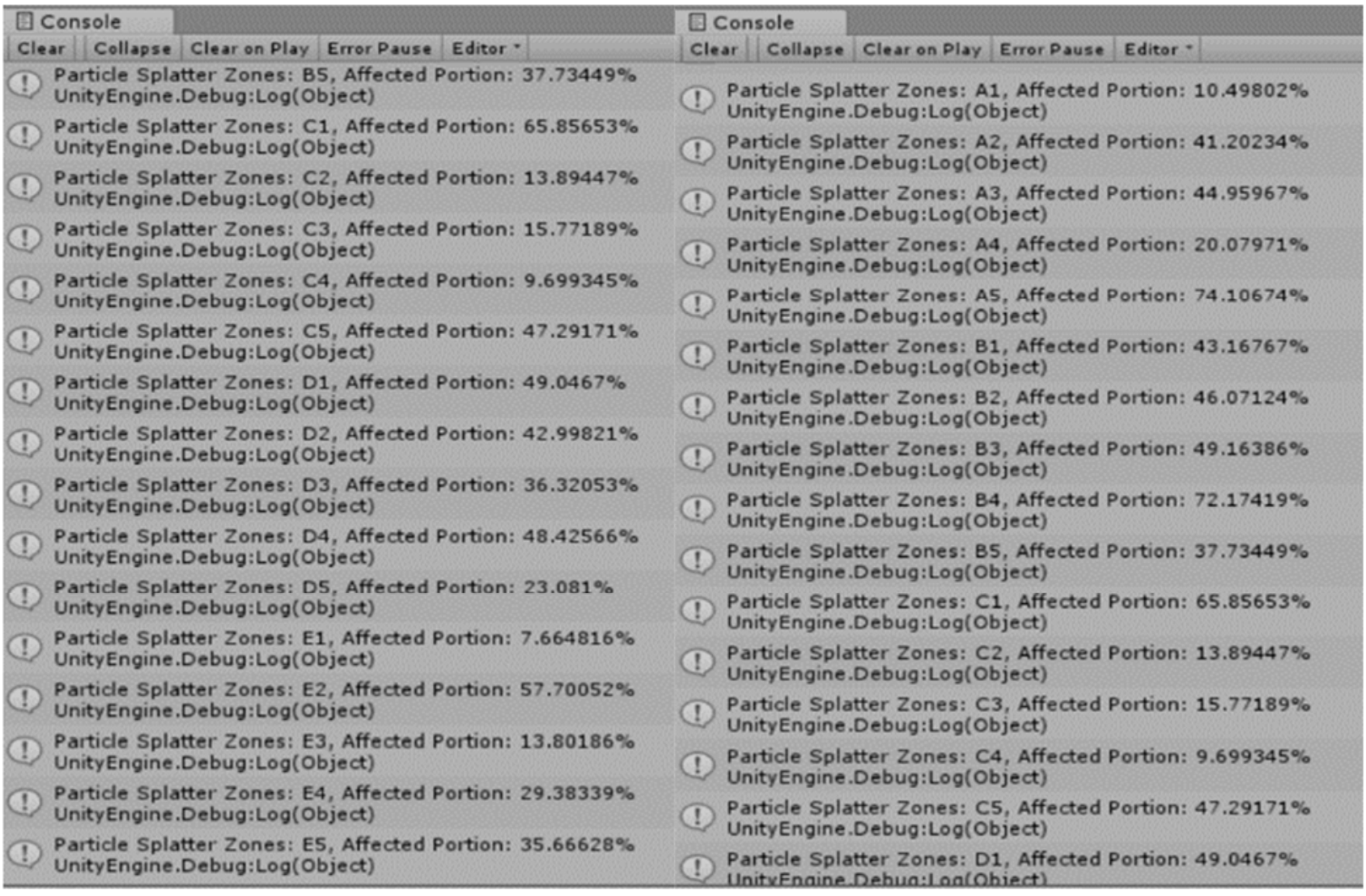

Figure 4.11 - Simulation console indicating the percentage of a sensor grid affected by particles.

Before each real shot, the pellet gun's projectile muzzle velocity is measured by the pellet chronographer. This doppler radar velocity sensor provides a highly-accurate measurement of the 
target's velocity. We vary the projectile velocity and the impact point of the target in our experiments. 


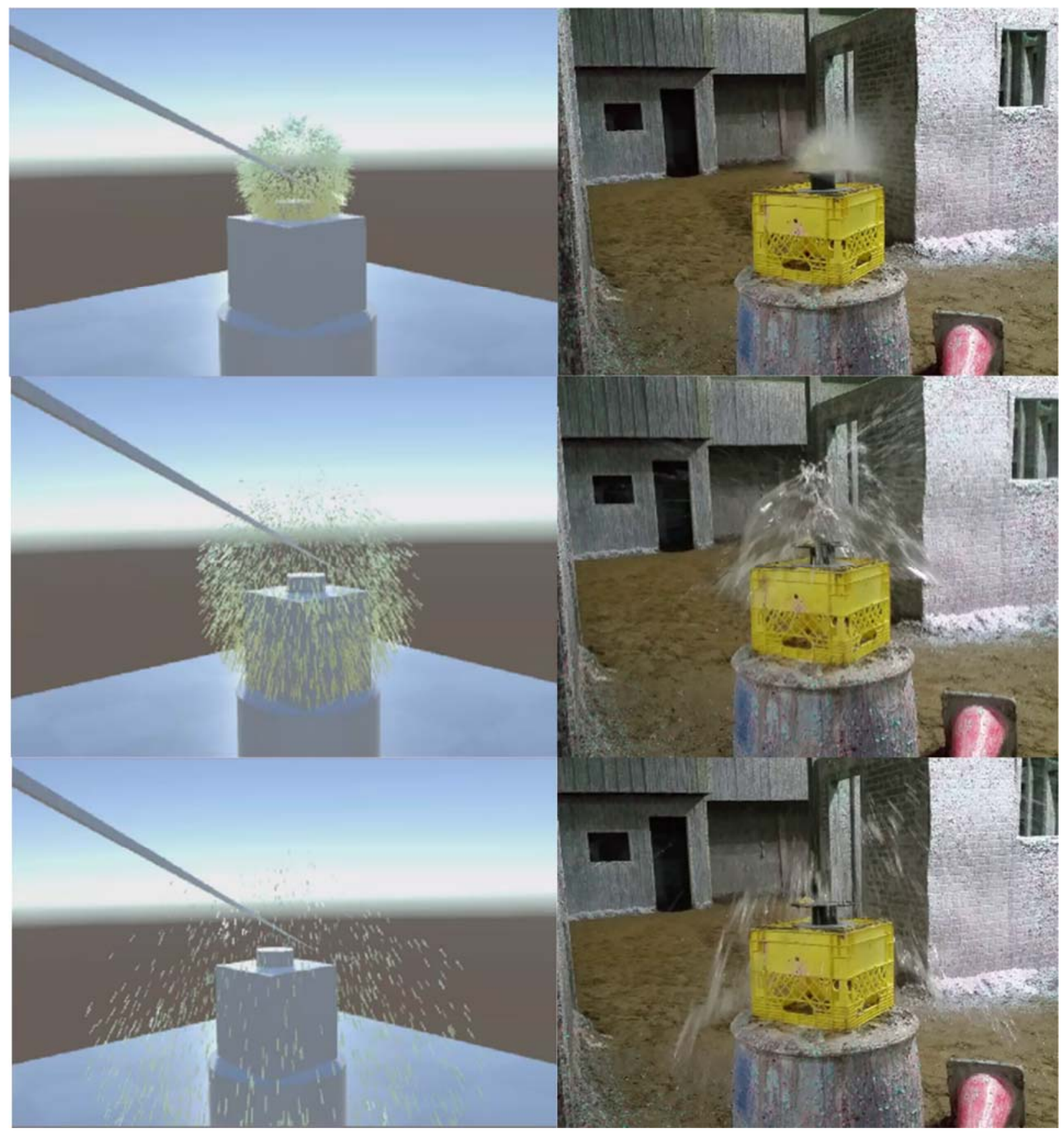

Figure 4.12 - Left: Simulation of a particle dispersion in action. Right: Real-world experiment of projectile impact resulting in dispersion.

The simulation correctly models a visual cue commonly associated with high-speed projectile impact with containers of liquid and causes a phenomenon called the 'bowl effect'. This 
visual effect is a combination of a shock wave propagating forwards into the liquid, and cavitation and possible air entrainment in the base/wake region of the projectile [92], [95].

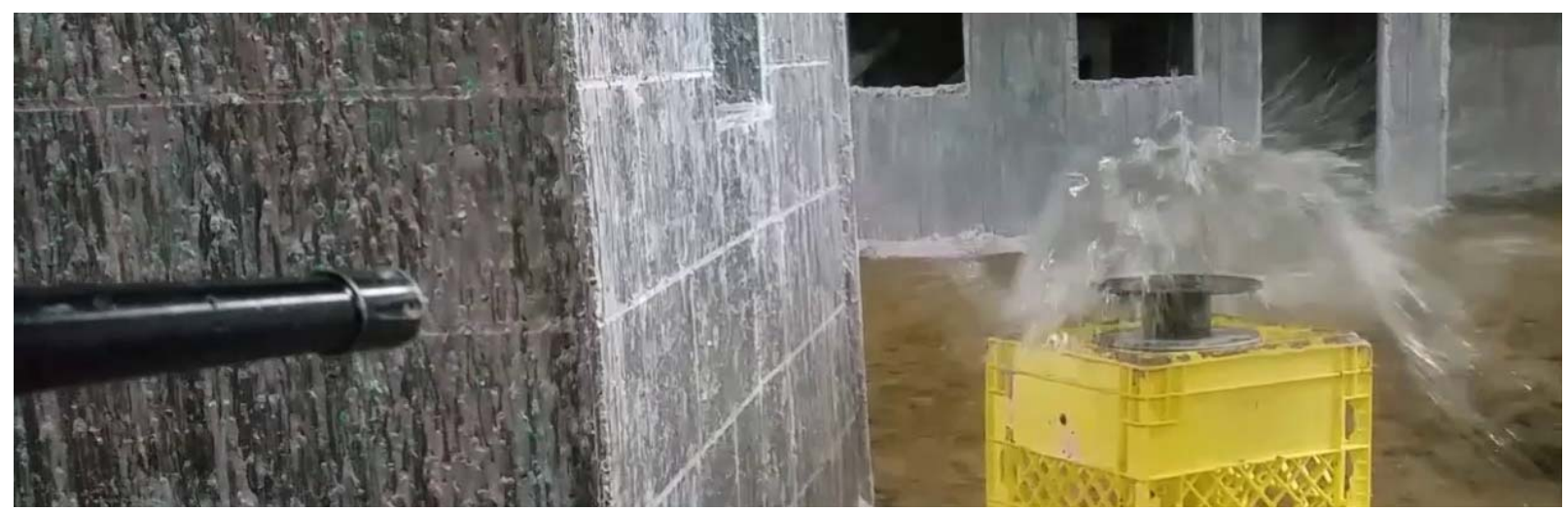

Figure 4.13 - The 'bowl effect' visual cue from a projectile impact.

Results are evaluated by comparing the percentages that the real-world sensor pad's grid is affected by splatter to the simulation's console output, and assuming that all factors are modelled correctly.

\begin{tabular}{|l|l|l|l|}
\hline Grid & Portion of Grid Affected (\%) & Console Output (\%) & Absolute Difference \\
\hline A1 & 15.78 & 37.49 & 21.70 \\
\hline A2 & 53.85 & 2.83 & 51.01 \\
\hline A3 & 2.58 & 48.25 & 45.67 \\
\hline A4 & 24.85 & 28.08 & 3.22 \\
\hline A5 & 63.23 & 3.48 & 59.74 \\
\hline B1 & 40.42 & 12.40 & 28.02 \\
\hline B2 & 66.15 & 11.21 & 54.94 \\
\hline B3 & 72.92 & 13.79 & 59.13 \\
\hline B4 & 61.52 & 4.52 & 57.00 \\
\hline B5 & 36.84 & 22.96 & 13.88 \\
\hline C1 & 57.35 & 0.65 & 56.71 \\
\hline
\end{tabular}




\begin{tabular}{|l|l|l|l|}
\hline C2 & 61.57 & 8.99 & 52.58 \\
\hline C3 & 33.94 & 25.76 & 8.18 \\
\hline C4 & 16.06 & 37.13 & 21.07 \\
\hline C5 & 53.97 & 0.09 & 53.87 \\
\hline
\end{tabular}

Table 4.10 - A view of the results comparing the percentages from the actual experiment and the virtual model.

The lower the percentage in the difference column, the more the results suggest that the simulation may accurately model a similar real-world system. On average, there is a $34.34 \%$ difference from 150 grid comparisons. We suggest that less than $50 \%$ difference is a sufficiently promising first step toward the possibility of producing accurate, discrete particle simulations of high-velocity projectile impacts on modeled IED objects of varying densities, masses and configurations.

We also note that the simulation uses perfectly spherical 3D virtual water balloons (computer generated models) - we did not utilize our CT scanner to scan water balloons so the exact dimensions differ from the actual dimensions of the water balloon - this factor may, in turn, affect the accuracy of our dispersion results. In the next section, we will discuss real-world testing of a disrupter shot at multiple objects that are scanned and have their 3D virtual model equivalents imported into our serious game simulation for analysis.

\subsubsection{Multiple Projectiles to Multiple Masses}

The next step is to test the simulation results using a real disrupter shot (multiple projectiles) travelling at $465 \mathrm{~m} / \mathrm{s}$ at multiple objects with varying densities and varying placements of target objects. When EDU prepare a disrupter cannon for firing the process is a bit of an art form as different power loads can be used with differing amounts of gun powder in them. EDU must select different loads to achieve different penetrations and dispersal - using the maximum 
propellant load may result in low dispersion/high accuracy, using less propellant may result in high dispersion/low accuracy and possibly partial penetration.

Our previous single projectile shot to one mass was simulated with a computer generated water balloon model that was perfectly spherical. In this section we test our simulation with actual CT scanned 3D virtual models, and use the exact real-world models in our experiments. With the assistance of EDU specialists [102], we were able to test our experiments with a real-world disrupter cannon and we compare the real-world splatter with its simulated analog generated with our dispersion model.

For our experiments, EDU specialists used a 29mm Neutrex waterjet disrupter cannon from Proparms Ltd and a consistent propellant load (75 rated gun powder).

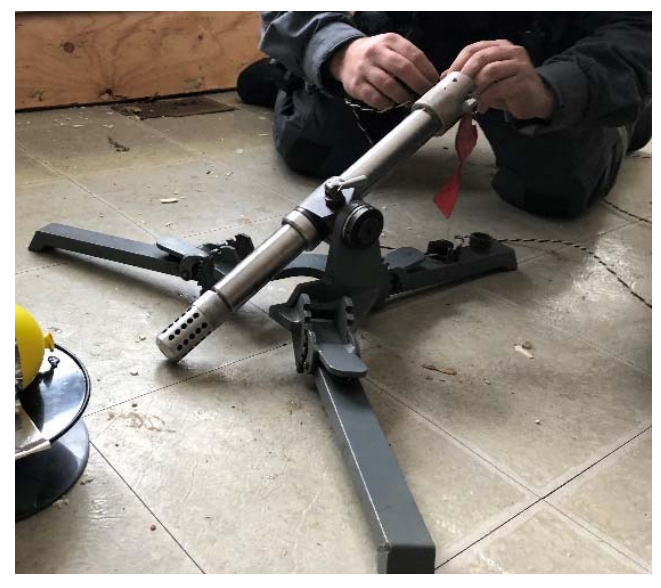

Figure 4.14 - A 29mm Neutrex waterjet disrupter cannon used for our real-world experiments.

The technical information of the $29 \mathrm{~mm}$ Neutrex waterjet disrupter cannon that we used is shown below: 
Total weight:

Peak liquid projectile velocity at muzzle:

Kinetic energy delivered on target by liquid projectile

Maximum recoil impulse:

\author{
$4.4 \mathrm{~kg}(9.7 \mathrm{lbs}$. \\ $465 \mathrm{~m} / \mathrm{s}(1,525 \mathrm{ft} / \mathrm{s})$ \\ $15.2 \mathrm{~kJ}$ \\ 88.4 N.s
}

The only information that is used in our simulation is the projectile muzzle velocity and mass of the projectile. Future work in our simulation may include factors such as kinetic energy as stated by its technical information, but is beyond the scope of this thesis, as well as the fact that different disrupters exist with significantly different properties. In theory, all these factors can be and should be implemented in the dispersion modelling process of an open system.

However, we assert that the methodology in 3.4 is adequate for a relatively accurate dispersion of particles as a result of a high powered projectile impact.

Our test method is as follows:

1. Obtain reasoning regarding pose and disrupter pose and direction from the serious game simulation regarding the disruption and dispersion of target models.

2. Find a suitable environment (indoor environment to reduce external environmental factors). EDU specialists will select an appropriate environment for conducting these experiments).

3. Setup HD cameras in multiple positions behind and on the side of the experiment, or wherever possible, but it is best to have at least one HD camera behind the firing apparatus - and configure cameras to record at 240 frames per second.

4. Setup disrupter apparatus, load disrupter with $200 \mathrm{ml}$ of water as the projectile (our test was done in winter, so we used windshield water fluid to withstand freezing temperatures, details of the disrupter cannon is in section 2.2.3). 
5. Arm disrupter with propellant charge (EDU specialists will perform this part).

6. Setup target objects and target according to intelligence obtained (pose of objects) from the game simulation - verify with EDU specialists that the target component is a key component of interest.

7. Setup disrupter and disrupter apparatus according to the simulation's specified pose and direction.

8. Initiate HD cameras and ensure all prior target objects pose and densities are recorded - at this stage the 3D virtual model should have been created already and tested in the game simulation.

9. Follow instructions from EDU specialists for the triggering and firing procedure of the disrupter - which is to clear the area and cover your ears during the firing process.

10. Follow instructions from EDU specialists for safe entry into the hot zone (area where it is dangerous for people to be during a disrupter shot).

11. Inspect sensor grid and remove it for evaluation.

12. Repeat steps 4-11, and replace the sensor grid for each experiment.

13. Visually inspect the sensor grid and record the percentage of affected grid area and compare results with in-game results.

The sensor grid pad we used to measure the dispersion splatter was an 11 by 18 sensor grid pad with each pad measuring $2 \times 2$ inches. For the sake of consistency and simplicity, we overlaid the $8 \times 11 \frac{1}{2}$ sensor grid pad used in our first test method and separated the 11 by 18 grid to match a 5 by 5 grid - doing this ensured that the no additional modifications were needed to be made in 
our game simulation. Splatter impact is visually estimated by the percentage of disrupted surface area. Results outputted in our simulation show grids A1-A5, B1-B5, ... to E1-E5 (25 in total).

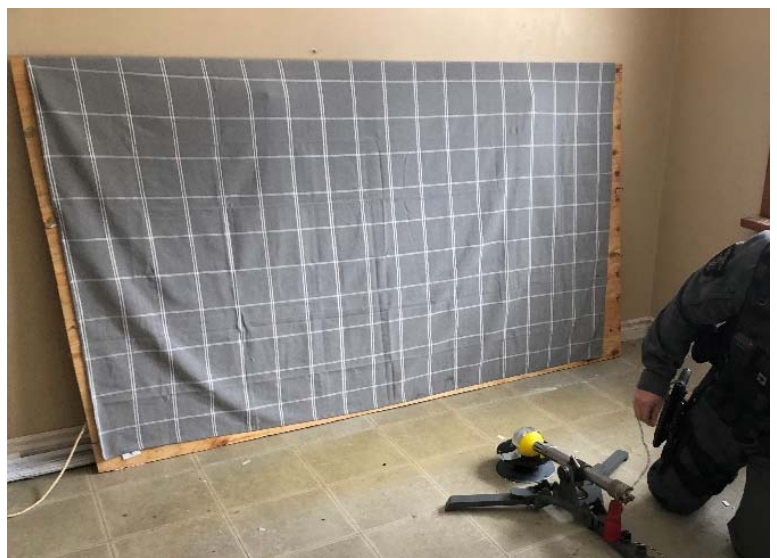

Figure 4.15 - An 11 by 18 sensor grid pad used to capture the percentage of splatter on each grid after a dispersion of components from a target.

We performed our real-world tests on the following targets:

1 water balloon with the disrupter targeting the center of the water balloon.

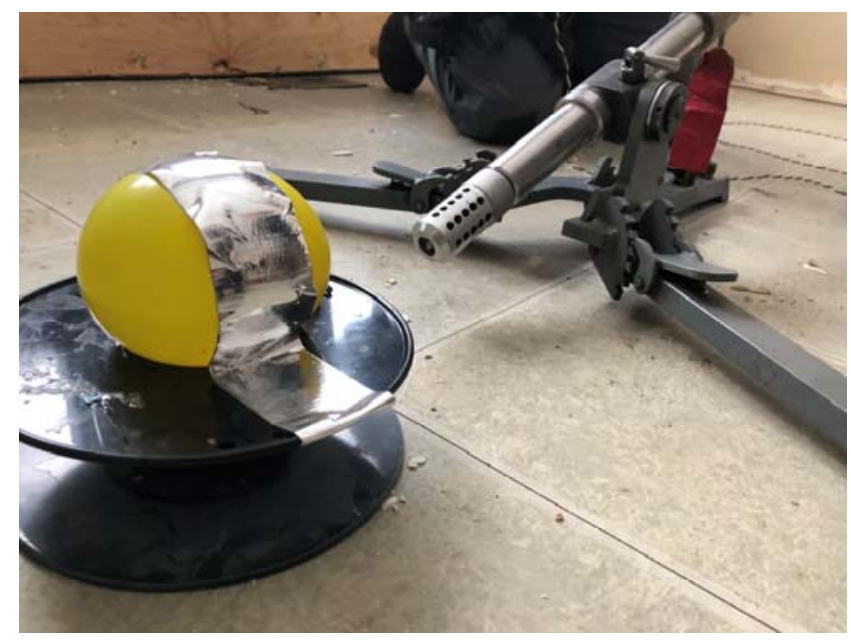

Figure 4.16 - Real-world experiment of a disrupter cannon targeting a water balloon. 
2 water balloons positioned side by side, with the disrupter targeting the centre of the two.

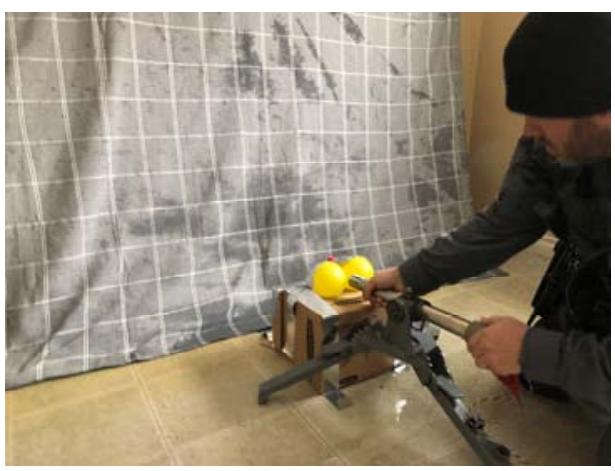

Figure 4.17 - Real-world experiment of a disrupter cannon targeting two water balloons.

A half full windshield water fluid in its container which is placed within a banker box - the disrupter targeting the exterior of the banker box.

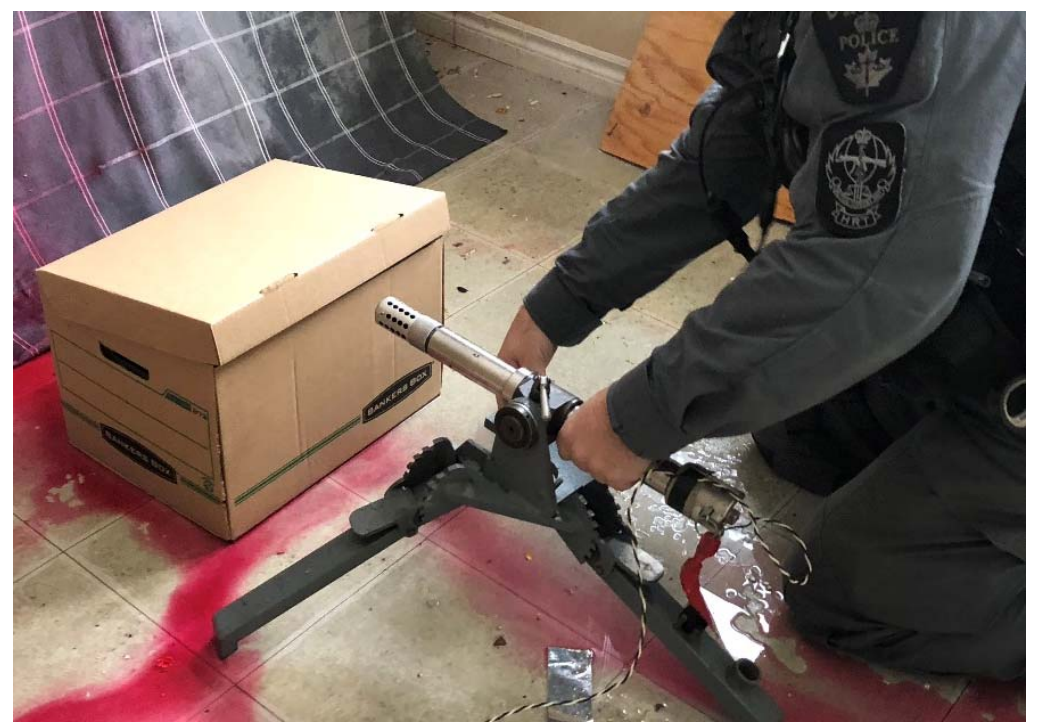

Figure 4.18 - Real-world experiment of a disrupter cannon targeting an occluded half-full windshield water container. 
Seven common substances, a frozen water bottle, a computer power supply, a water balloon and an inert nitro dynamite - the disrupter targeting circuitry attached to the power supply.

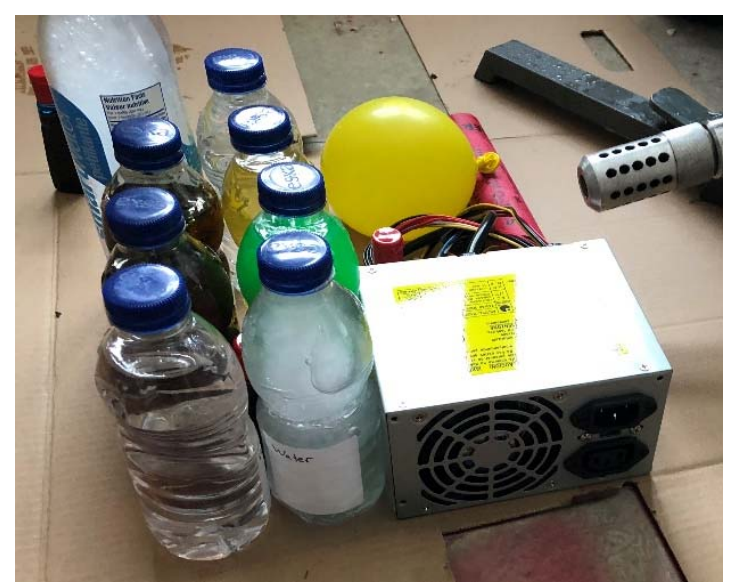

Figure 4.19 - Real-world experiment of a disrupter cannon targeting multiple objects, one of which is an inert nitro dynamite.

The results are shown in Table 4.11, we averaged each the results of every 5 grids and merged it into one grid, the middle column outputs the simulation console output, and the last column outputs the absolute difference.

\begin{tabular}{|l|l|l|l|}
\hline Target: 1 water balloon & Console Output (\%) & Abs Difference \\
\hline Grid Section & Portion of Grid Affected (\%) & 26.43 & 11.57 \\
\hline A & 38 & 37.23 & 16.77 \\
\hline B & 54 & 54.21 & 15.79 \\
\hline C & 70 & 43.03 & 14.97 \\
\hline D & 58 & 3.50 & 11.50 \\
\hline E & 15 & \multicolumn{3}{l|}{} \\
\hline Target: 2 water balloons & 86.67 & 10.33 \\
\hline A & 97 & 35.69 & 12.31 \\
\hline B & 48 & 29.59 & 11.41 \\
\hline C & 41 & 56.78 & 14.22 \\
\hline D & 71 & 17.04 & 17.96 \\
\hline E & 35 & \multicolumn{3}{l|}{} \\
\hline Target: A half full windshield water container & 79.48 & 19.52 \\
\hline A & 99 & 13.88 & 11.88 \\
\hline B & 2 & \multicolumn{2}{|l|}{} \\
\hline
\end{tabular}




\begin{tabular}{|l|l|l|l|} 
C & 59 & 48.56 & 10.44 \\
\hline D & 90 & 72.31 & 17.69 \\
\hline E & 94 & 74.14 & 19.86 \\
\hline $\begin{array}{l}\text { Target: Seven common substances, a frozen water bottle, a computer power supply, a water balloon and an inert } \\
\text { nitro dynamite. }\end{array}$ & 3 & 11.48 & 8.48 \\
\hline A & 46 & 32.03 & 13.97 \\
\hline B & 13 & 6.08 & 6.92 \\
\hline C & 71 & 56.11 & 14.89 \\
\hline D & 16 & 0.70 & 15.30 \\
\hline E & & & \multicolumn{2}{l}{} \\
\hline
\end{tabular}

Table 4.11 - Validation results of the real-world test to the simulation's output. The rightmost column shows the difference between the two results.

From Table 4.11, we observe that the average difference between the real-world experiment and the virtual experiment is $14.41 \%$. This validation result is below our self-proposed acceptable difference of $50 \%$.

We note two distinctions from our single projectile test; first, the virtual models are created using our 3D model creation method as stated in section 3.2 in which we use a CT scanner to acquire data and various pre-processing methods to create a 3D virtual model. Second, we adjusted our real-world experiment to match the simulation's reasoning regarding the target object's pose and the disrupter's pose and direction. This information suggests that the reasoning obtained from the serious game simulation is a step towards providing relatively accurate splatter impact points as long as accurate 3D virtual models (programmed with relevant characteristics) are part of the simulation and are used in calculating dispersion.

We have developed a methodology that suggests an open system game simulation may be useful in planning the disruption of real-world IEDs, in which game actors can position and target objects with a projectile of user-defined velocity, acceleration, mass and density. This approach 
permits faithful reproduction of static and dynamic particle interactivity and may be tunable to emulate real-world effects without danger to the public or first responders.

This work stems from our previous findings that enable us to determine relevant physical characteristics of unknown objects, with results that are obtained and verified from similar explosive analogs [22]. The work was informed by our previous findings that enable us to compute and simulate spatial shrapnel impact points from a directionally focused charge calculated using flow topology algorithms [23].

We argue that this methodology is relevant and can be accurately used to analyze particle dispersion for the purposes of augmenting IED neutralization processes by using serious game techniques.

Our methodology allows for the incorporation of additional factors such as elasticity, dampening, and other forces, allowing for highly-dynamic particle interaction. However, this simulation only models dispersions, which assumes that the game actor has successfully dynamically interacted with critical components of the IED without setting off its explosive charge(s).

\subsubsection{Analysis of Numerical Results}

The targeting of the disrupter cannon crosshair in the simulation significantly affects the console output that shows the affected percentage of the grid. To ensure we positioned the disrupter cannon and its direction appropriately, we matched the real-world exact pose and angle of the cannon to the simulation's exact pose and angle of the cannon. To do that, prior to each experiment, we measured the distance from the disrupter cannon at least 3 times with a distance measuring tool 
(Fnova Laser Distance Measure) and an angle measuring tool (Instruments 836 Angle-Izer in 3 planes). In our simulation, we matched the distance/angles of the cannon to the target to the tenth degree.

The grids that were mostly affected were C, D and E, as these grids capture splatter relatively lower on the sensor pad. The portion of the grid that was affected that had percentages ranging from $1-30 \%$ and $70 \%-99 \%$ matched closely to the simulation's analysis of splatter impact points. There is no visualization from the sensor grid shown in the simulation, as the calculation of the particle dispersion model of a successful IED neutralization takes about 3-4 hours to compute, with an update of 1 frame per second. We relied on the dispersion model's console output, and to decrease lag and computation power, the sensor grid in the simulation is transparent. The portion of the grid that was affected that had percentages ranging from $30 \%-70 \%$ show more variation.

We argue that this analysis is relevant for showing minor and major impact splatter points - and the major points of impact may be significant in EDU neutralization processes. However, further work is yet to be done in visualizing the simulation splatter without significant delay due to computation time, and the consideration of additional factors in an open system neutralization process. 


\section{Chapter 5 Conclusion and Future Work}

\subsection{Summary of Results}

In this thesis, we developed an algorithm with the goal of providing EDU with additional information to aid in the IED neutralization process. The information consist of the possible type of explosive employed in an IED, the potential major points of impact (splatter) as a result of the physical dispersion of the components of an IED, and any and possible actionable intelligence on the pose and direction of a disrupter cannon of a successful IED neutralization.

We have developed a prototype simulation based on this algorithm and evaluated the simulation with an appropriate real-world disrupter and compared the real-world splatter to our simulation's splatter.

We argue systems developed with our algorithm may provide relevant information directly from the simulation and can be accurately used to analyze particle dispersion for the purposes of augmenting EDU IED neutralization processes.

We argue we addressed this challenge by providing a purpose-built serious game simulation that provides a mechanism for EDU members to practice with, identify critical components of interest, interact with virtual IEDs, become aware of range of the type of explosive, obtain objective scores according to their performance in the game and understand potential primary and secondary spatial impact points concerning the IED neutralization task.

Our simulation games provide accurate and familiar analogs to various activities and objects from the real-world, like aiming and manipulating a disrupter and the IED itself, in the form of a game for the purposes of analysis, and prediction. 
An IED presents many challenges such as composition of unknown elements, ranges in form, size and power, and its placement in an innocuous container, whereby the spatial arrangement, density, and mass of the IED components are unknown.

We addressed these challenges with an algorithm that has the following objectives;

1. Provide a physically accurate representation of an IED that maintains all spatial relationships between its components.

2. Provide information regarding a range of the type of explosive(s) employed in the device and ascertain to a degree of certainty the nature of the unknown materials in a concealed container.

3. Provide splatter impact points and its primary and secondary effects on its surroundings as a result of an effective IED neutralization shot

4. Provide potentially actionable intelligence such as objective scoring, neutralization cannon pose and direction, reasoned density estimate of unknown occluded target objects, and splatter impact points on the surrounding environment to EDU.

Our contributions are as follows:

1. An augmented method for scanning inert IED models using a 3D scanner. (Addresses challenge \#1)

2. An augmented method to create 3D virtual IED models. (Addresses challenge \#1)

3. A method to determine the range of the type of explosive employed in an IED that is hidden in a package. (Addresses challenge \#2) 
4. An augmented method to disintegrate 3D virtual IED components into parts (or particles) with specific characteristics of its real-world material. (Addresses challenge $\# 1)$

5. Demonstration of complex model interactions within serious game simulation and reasoning as a result of these complex model interactions. (Addresses challenge \#4)

6. Particle dispersion as a result of a high velocity projectile impact methodology. (Addresses challenge \#3)

The result of our algorithm is our proof of concept system, which is a serious game IED neutralization system. This serious game has a game actor capable of manipulating accurate 3D virtual IED models and neutralizing the IED with a disrupter cannon, and produces possible actionable intelligence relevant for EDU neuralization processes.

In our experimental results, we claim that we proved that our algorithm can provide accurate 3D models of IEDs, provide relevant information regarding the possible type of explosive employed in the device, and provide accurate splatter impact points as a result of a successful neutralization shot through model validation and splatter impact point validation methods.

In model validation, we verified the dimension, mass and density of our virtual 3D models with real-world models and deemed our results to match real-world characteristics with acceptable degrees of discrepancy.

In splatter impact point validation method, we verified our game-based simulation splatter impact points to real-world splatter impact points using a paintball gun and an actual disrupter cannon. 
Armed with the capability to safely analyze real-world bombs, we have provided evidence that supports the assertion that our system can aid EDU operations. The ability to interact with 3D virtual models will potentially provide additional IED awareness and support the decision making process of IED neutralization.

In summary, the system's algorithm is split into 4 phases, they are as follows;

Phase 1 - the creation of mock IEDs, calibrating sensors, acquiring and analyzing data. (Contribution \#1, \#3)

Phase 2 - the creation, cleanup and segmentation of virtual models and meshes, and associating bounding boxes. (Contribution \#2, \#4)

Phase 3 - the implementation of molecular dynamics, real-world physical attributes, projectile, dispersion to models, and implementation of the game actor to its associated real-world traits. (Contribution \#5, \#6)

Phase 4 , the testing and validation of the game. (Validation of our contributions).

Figure 3.1 shows an overview of our algorithm. The system begins with gathering input, which is cross-sectional DICOM data of real-world inert IEDs and inert explosives collected from a CT machine. The cross sectional data are merged to create a 3D model, which is then modified and segmented to separate key IED components of interest, and the assemblage of these components are imported into a game engine, where bounding boxes, scoring criteria, real-world physical attributes are associated with each component, and each component is programmed with dispersion model mechanics that adhere to molecular dynamics. Last, the reasoning from our 
serious game is tested with an actual Proparms disrupter cannon on actual targets, and the serious game is tested by an EDU specialist and a Proparms technician.

\subsection{Key contributions of this thesis}

We developed a method to determine the range of the type of explosive employed in an IED that is hidden in a package. To do this, we have proposed a methodology to calibrate a CT scanner and ascertain to a relative degree of certainty, the relative density of the unknown materials in a concealed container. And we argue that this information is a useful for threat-detection personnel. This methodology can be used as long as subsequent scans use the same predefined parameters, procedure and protocol and scanning machine.

In addition, we also developed a method to disintegrate 3D virtual IED components into parts (or particles) with specific characteristics of its real-world material. Each particle is programmed with characteristics of its real-world analog and subject to 3-dimensional physics when external forces are applied.

Another notable contribution is our dispersion methodology as a result of a high velocity projectile impact methodology. We developed this methodology to compute and simulate dispersion and collision effects of particles as a result of a high velocity projectile impact on a container with varying density particle models enclosed. 


\subsection{Limitations and Future Work}

The system can be considered a proof of concept to aid EDU operations but is not without its drawbacks and limitations. As discussed in our previous work [29], conventional CT scanners are extremely large and near-impossible to relocate if needed. However, portable or mobile CT scanners are becoming more commonplace [125] a solution designed for mobile healthcare environments such as military intensive care units, but may be useful in EDU processes.

CT scanners provide an ability to output multiple tomographic images (useful for 3D model reconstruction and analysis) but their drawbacks are colourless images, misrepresentative artifacts caused by high density materials (like metal), and as discussed briefly in Chapter 3, inaccurate relative densities of very fine particles (aka powder). Powder such as smokeless black powder and PETN include pockets of air during a CT scan. Therefore, the system is unable to accurately determine the range of the type of explosive in the unknown substance, however, future work may involve scanning a large selection of inert and real explosives to such a degree that will allow a very accurate estimation of the possible type of explosion concealed in a container. There is further work to be done in research for an algorithmic way of exactly identifying a property that suggests, or even indicates the presence of an explosive, rather than relying on canines to indicate the presence of explosives.

Despite these drawbacks, CT scanners provide highly accurate surface models of interior and exterior materials and with a high degree of fidelity even when materials of different densities are scanned at the same time. X-ray scanners, on the other hand, provide little to no distinct object occlusion differentiation due to its projected 2D image (it is difficult to discern whether an object is in front of or behind another). 
Our approach is not limited to the use of a CT scanner, as long as the theory behind our algorithm is followed. We suggest that $3 \mathrm{D}$ virtual models can also be created, programmed to have real-world physical characteristics and also manipulated and output reasonably accurate real-world interactions.

Within our approach, human operators are required to determine key critical components of an IED, failure to determine these components correctly will result in incorrect feedback from the simulation. Human error can so occur in deciding CT scanning parameters, model creation using signal thresholds, model cleanup or associating bounding boxes and their score. However, the claim still remains that the algorithm can create a serious game simulation and is a safe environment for virtual IED interaction.

Currently our proof of concept system utilizes a keyboard and mouse as user interfaces these are not representative of the real-world. However, future work may include importing the system into an immersive serious game system which utilize virtual headsets and hand sensors.

The advantage in using our proposed algorithm is that the identification process to identify bomb neutralization targets in virtual IEDs can be easily shared between EDUs throughout the world. A database of 3D virtual IED models and procedural logs from the serious game simulation can be shared with EDU facilities - which can possibly enable a standardized regimen for EDU and allow expert EDU to improve or maintain their knowledge with a database of 3D virtual IED models.

For the moment, the use of medical imaging technology for IED neutralization processes is beyond the capabilities of most EDUs who are only equipped with x-ray equipment as of our 
previous work [29]. However, the process of calibrating the scanner can theoretically be used for other scanning technology to determine relative densities of unknown substances in the target scan. The use of a 3D scanner reveals information that may be affected by occlusions when using a $2 \mathrm{D}$ scanner, however, future work may involve the use of computer vision techniques to utilize multiple 2D images to create a 3D model.

Another future work that may be of interest is to take the human involvement elements out of the algorithm and possibly automate the neutralization simulation process from start to end.

The threat of IEDs is present, and IED related incidents may occur anywhere resulting in potentially massive destruction to property, injury and death. IEDs are becoming more lethal and is commonly used by insurgents, which calls for an increasing demand for counter-IED measurements. In this thesis we suggested augmented scanning technology and additional intelligence from a serious game that may aid in the IED neutralization process, but there are still plenty of challenges that are still unaddressed or need improvement, we hope that this thesis can point towards future research in this area that may affect millions of lives. 


\section{Appendix}
A. Mesh Chunk Splitting Algorithm

public Mesh[] GetMeshChunks(int width, int length, int splits)

\{

int chunkWidth = width / splits;

int chunkLength = length / splits;

Mesh[] meshes = new Mesh[splits * splits];

Mesh chunk = new Mesh();

List $<$ Vector $3>\mathrm{cV}=$ new List $<$ Vector $3>($ )

List $<$ Vector $2>$ cUV $=$ new List $<$ Vector $2>($ );

List $<$ int $>$ cT $=$ new List $<$ int $>($ );

for (int I = $0 ; \mathrm{I}<$ chunkLength; $\mid++$ )

\{

for (int $v=0 ; v<=$ chunkWidth; $v++$ ) \{

int $\mathrm{i}=(\mathrm{I} *$ width $)+\mathrm{v}$;

cV.Add(verts[i]);

cUV.Add(uvs[i]);

\}

if (I >= chunkLength - 1 ) continue;

for (int $\mathrm{t}=0 ; \mathrm{t}<$ chunkWidth *6; $\mathrm{t++}$ ) \{

int $\mathrm{i}=\left(I^{*}(\right.$ width -1$\left.) * 6\right)+\mathrm{t}$;

cT.Add(tris[i]);

\}

\}

chunk.vertices $=c$ V.ToArray () ;

chunk.triangles = cT.ToArray ();

chunk.uv = cUV.ToArray();

chunk.RecalculateBounds();

chunk.RecalculateNormals();

meshes[0] = chunk;

return meshes;

\} 


\section{Bibliography}

[1] T. Collins, "Manchester bombing: don't blame the New York Times for printing leaked information," The Conversation, 2017.

[2] G. M. Muhl Jr, "Defeating Improvised Explosive Devices (IED): Asymmetric Threats and Capability Gaps," ARMY WAR COLL CARLISLE BARRACKS PA, 2011.

[3] S. Hali, "IEDs: bane for humanity," Defence Journal, vol. 16, no. 1/2, p. F1, 2012.

[4] J. Dowle, "Improvised explosive devices," Law and Order, vol. 54, no. 9, pp. 28-31, 2006.

[5] A. Ramasamy, S. E. Harrisson, J. C. Clasper, and M. P. Stewart, "Injuries from roadside improvised explosive devices," Journal of Trauma and Acute Care Surgery, vol. 65, no. 4, pp. 910-914, 2008.

[6] D. C. Goodrich and F. L. Edwards, "Improvised Explosive Devices," Crisis and Emergency Management: Theory and Practice, pp. 178-189, 2014.

[7] N. Youngblood, The development of mine warfare: a most murderous and barbarous conduct. Greenwood Publishing Group, 2006.

[8] G. H. Alexander, J. T. Stroup, J. P. LaBine, and B. G. DeRoos, "Explosives disrupter." Google Patents, Dec-2002.

[9] P. Weiss, "Device for disrupting improvised explosive devices (IEDS)." Google Patents, Jan-2009.

[10] D. Attwood and A. Sakdinawat, X-rays and extreme ultraviolet radiation: principles and applications. Cambridge university press, 2017.

[11] J. Hsieh and others, "Computed tomography: principles, design, artifacts, and recent advances," 2009.

[12] M. Graafland, J. M. Schraagen, and M. P. Schijven, "Systematic review of serious games for medical education and surgical skills training," British journal of surgery, vol. 99, no. 10, pp. 1322-1330, 2012.

[13] D. Varas, J. López-Puente, and R. Zaera, "Numerical analysis of the hydrodynamic ram phenomenon in aircraft fuel tanks," AIAA journal, vol. 50, no. 7, pp. 1621-1630, 2012.

[14] N. Bell, Y. Yu, and P. J. Mucha, "Particle-based simulation of granular materials," in Proceedings of the 2005 ACM SIGGRAPH/Eurographics symposium on Computer animation, 2005, pp. 77-86.

[15] Philips, "Philips - Pinnacle ${ }^{3}$ Auto-Planning." .

[16] J. Webster, Medical instrumentation: application and design. John Wiley \& Sons, 2009.

[17] F. E. Boas and D. Fleischmann, "CT artifacts: causes and reduction techniques," Imaging Med, vol. 4, no. 2, pp. 229-240, 2012.

[18] M. Macklin, M. Müller, N. Chentanez, and T.-Y. Kim, "Unified particle physics for realtime applications," ACM Transactions on Graphics (TOG), vol. 33, no. 4, p. 153, 2014.

[19] A. Beveridge, "Improvised Explosive Devices," 2013.

[20] A. A. Faust, C. De Ruiter, A. Ehlerding, J. E. McFee, E. Svinsås, and A. D. Van Rheenen, "Observations on military exploitation of explosives detection technologies," in Detection and Sensing of Mines, Explosive Objects, and Obscured Targets XVI, 2011, vol. 8017, p. 801716.

[21] C. C. Chan, A. Ferworn, and C. M. Young, "Serious gaming for improvised explosive device neutralization training," in MATEC Web of Conferences, 2016, vol. 68, p. 02006. 
[22] C. C. Chan, A. Ferworn, and L. Chin, "Towards determining relative densities for common unknown explosives in improvised explosive devices," in Humanitarian Technology Conference (IHTC), 2017 IEEE Canada International, 2017, pp. 55-60.

[23] C. C. Chan, A. Ferworn, and D. Tran, "A Rudimentary Approach to Unmanned Aerial Vehicle Guided Improvised Explosive Device Shrapnel Dispersal Simulation," in International Conference on Intelligent Networking and Collaborative Systems, 2017, pp. 108-118.

[24] H. Schubert and A. Kuznetsov, Detection and disposal of improvised explosives. Springer Science \& Business Media, 2006.

[25] P. D. Biddinger, A. Baggish, L. Harrington, P. d Hemecourt, J. Hooley, J. Jones, R. Kue, C. Troyanos, and K. S. Dyer, "Be prepared - the Boston Marathon and mass-casualty events," New England journal of medicine, vol. 368, no. 21, pp. 1958-1960, 2013.

[26] A. Farazmand, Handbook of crisis and emergency management. CRC Press, 2001.

[27] V. Smirnov, "Possibilities of Creating Improvised Explosives and Explosive Devices," in Detection and disposal of improvised explosives, Springer, 2006, pp. 217-221.

[28] D. Hogan, "Physical injuries and fatalities resulting from the Oklahoma City bombing," Jama, vol. 276, pp. 382-387, 1996.

[29] C. Chan, "GAME-BASED THREAT ASSESSMENT TOOL FOR IMPROVISED EXPLOSIVE DEVICE NEUTRALIZATION TRAINING," 2015.

[30] D. F. Eisler, "Counter-IED strategy in modern war," Military Review, vol. 92, no. 1, pp. 9$15,2012$.

[31] L. Weiss, E. Whitaker, E. Briscoe, and E. Trewhitt, "Evaluating counter-IED strategies," Defense \& Security Analysis, vol. 27, no. 2, pp. 135-147, 2011.

[32] J. Allison, "Terrorist Weapons and Technology," Combating Terrorism in Northern Ireland, pp. 102-127, 2009.

[33] C. Wilson, "Improvised explosive devices (IEDs) in Iraq and Afghanistan: effects and countermeasures," 2007.

[34] R. Keeley, "Improvised Explosive Devices (IED): A Humanitarian Mine Action Perspective," Journal of Conventional Weapons Destruction, vol. 21, no. 1, p. 3, 2017.

[35] J. Revill, Improvised explosive devices: the paradigmatic weapon of new wars. Springer, 2016.

[36] C. J. Aylwin, T. C. König, N. W. Brennan, P. J. Shirley, G. Davies, M. S. Walsh, and K. Brohi, "Reduction in critical mortality in urban mass casualty incidents: analysis of triage, surge, and resource use after the London bombings on July 7, 2005," The Lancet, vol. 368, no. 9554, pp. 2219-2225, 2006.

[37] J. P. G. de Ceballos, F. Turégano-Fuentes, D. Pérez-Diaz, M. Sanz-Sanchez, C. MartinLlorente, and J. Guerrero-Sanz, "11 March 2004: The terrorist bomb explosions in Madrid, Spain-an analysis of the logistics, injuries sustained and clinical management of casualties treated at the closest hospital," Critical Care, vol. 9, no. 1, p. 104, 2004.

[38] C. Sloan, Mine warfare on land. Macmillan General Reference, 1986.

[39] B. M. Carnahan, "The law of land mine warfare: Protocol II to the United Nations Convention on Certain Conventional Weapons," Mil. L. Rev., vol. 105, p. 73, 1984.

[40] A. H. Cordesman and A. R. Wagner, "The Lessons of Modern War: Volume II," The IranIraq War, p. 1990, 1994. 
[41] J. Wyatt, "Land mine warfare: Recent lessons and future trends," International Defense Review, vol. 11, pp. 1499-1506, 1989.

[42] S. J. Whitney, J. J. Fidock, N. Ferguson, and others, "Assessing the effectiveness of simulation-based counter-IED training," Journal of battlefield technology, vol. 15, no. 1, p. 57, 2012.

[43] G. Zorpette, “Countering IEDs," IEEE spectrum, vol. 45, no. 9, 2008.

[44] C. Bruschini, "Commercial systems for the direct detection of explosives for explosive ordnance disposal tasks," Subsurface Sensing Technologies and Applications, vol. 2, no. 3, pp. 299-336, 2001.

[45] K. Wells and D. Bradley, "A review of X-ray explosives detection techniques for checked baggage," Applied Radiation and Isotopes, vol. 70, no. 8, pp. 1729-1746, 2012.

[46] A. K. Breed, H. Stoeckle, and D. Tuttle Jr, "Water cannon for neutralizing explosive devices, and replaceable cartridge therefor." Google Patents, Aug-1992.

[47] C. C. Mattern, "Cannon for disarming an explosive device." Google Patents, Apr-1998.

[48] T. E. Moutafis, C. R. Coffin, F. Van Patterson, and M. J. Yoder, "Fluid jet cutting system." Google Patents, Apr-2001.

[49] A. Tyas and J. A. Warren, "Detonation interrupter." Google Patents, Jul-2009.

[50] T. G. Brodie and A. W. Gleason, Bombs and bombings: A handbook to detection, disposal, and investigation for police and fire departments. CC Thomas, 1996.

[51] J. T. Bushberg, The essential physics of medical imaging. Lippincott Williams \& Wilkins, 2002.

[52] J. L. Prince and J. M. Links, Medical imaging signals and systems. Pearson Prentice Hall Upper Saddle River, 2006.

[53] M. Ellenbogen and R. R. Biijani, "System and method for CT scanning of baggage." Google Patents, May-2006.

[54] D. Koningsberger and R. Prins, "X-ray absorption: principles, applications, techniques of EXAFS, SEXAFS, and XANES," 1988.

[55] G. Zentai, "X-ray imaging for homeland security," International Journal of Signal and Imaging Systems Engineering, vol. 3, no. 1, pp. 13-20, 2010.

[56] U. Platt and J. Stutz, "Differential absorption spectroscopy," in Differential Optical Absorption Spectroscopy, Springer, 2008, pp. 135-174.

[57] S. U. Khan and W. Y. Chai, "An Image Enhancement Technique of X An Image Enhancement Technique of X-Ray Carry Ray Carry Ray Carry-on Luggage on Luggage for Detection of $\mathrm{C}$ for Detection of $\mathrm{C}$ for Detection of Contraband/ontraband/ontraband/Illicit," 2012.

[58] D. Mery, "Inspection of complex objects using multiple-X-ray views," IEEE/ASME Transactions on Mechatronics, vol. 20, no. 1, pp. 338-347, 2015.

[59] V. Rebuffel, J. Rinkel, J. Tabary, and L. Verger, "New perspectives of X-ray techniques for explosive detection based on CdTe/CdZnTe spectrometric detectors," in International Symposium on Digital Industrial Radiology and Computed Tomography-We, 2011, vol. 2, pp. 1-8.

[60] S. J. Kisner, "Image Reconstruction for X-ray Computed Tomography in Security Screening Applications," 2013.

[61] V. Riffo, S. Flores, and D. Mery, "Threat Objects Detection in X-ray Images Using an Active Vision Approach,” Journal of Nondestructive Evaluation, vol. 36, no. 3, p. 44, 2017. 
[62] W. Kalender, D. Felsenberg, O. Louis, P. Lopez, E. Klotz, M. Osteaux, and J. Fraga, "Reference values for trabecular and cortical vertebral bone density in single and dualenergy quantitative computed tomography.," European journal of radiology, vol. 9, no. 2, pp. 75-80, 1989.

[63] M. K. Kalra, M. M. Maher, T. L. Toth, B. Schmidt, B. L. Westerman, H. T. Morgan, and S. Saini, "Techniques and applications of automatic tube current modulation for CT," Radiology, vol. 233, no. 3, pp. 649-657, 2004.

[64] C. Levi, J. E. Gray, E. McCullough, and R. Hattery, "The unreliability of CT numbers as absolute values," American Journal of Roentgenology, vol. 139, no. 3, pp. 443-447, 1982.

[65] V. Riffo and D. Mery, "Automated detection of threat objects using adapted implicit shape model," IEEE Transactions on Systems, Man, and Cybernetics: Systems, vol. 46, no. 4, pp. 472-482, 2016.

[66] D. Mery, E. Svec, M. Arias, V. Riffo, J. M. Saavedra, and S. Banerjee, "Modern computer vision techniques for x-ray testing in baggage inspection," IEEE Transactions on Systems, Man, and Cybernetics: Systems, vol. 47, no. 4, pp. 682-692, 2017.

[67] D. Mery, G. Mondragon, V. Riffo, and I. Zuccar, "Detection of regular objects in baggage using multiple X-ray views," Insight-Non-Destructive Testing and Condition Monitoring, vol. 55, no. 1, pp. 16-20, 2013.

[68] D. S. Moore, "Instrumentation for trace detection of high explosives," Review of Scientific Instruments, vol. 75, no. 8, pp. 2499-2512, 2004.

[69] I. Lewis, N. Daniel Jr, N. Chaffin, P. Griffiths, and M. Tungol, "Raman spectroscopic studies of explosive materials: towards a fieldable explosives detector," Spectrochimica Acta Part A: Molecular and Biomolecular Spectroscopy, vol. 51, no. 12, pp. 1985-2000, 1995.

[70] R. M. Wentworth, J. Neiss, M. P. Nelson, and P. J. Treado, "Standoff Raman hyperspectral imaging detection of explosives," in Antennas and Propagation Society International Symposium, 2007 IEEE, 2007, pp. 4925-4928.

[71] W. M. Lagna, "Stand-off chemical detector." Google Patents, Feb-2012.

[72] J. C. Carter, S. M. Angel, M. Lawrence-Snyder, J. Scaffidi, R. E. Whipple, and J. G. Reynolds, "Standoff detection of high explosive materials at 50 meters in ambient light conditions using a small Raman instrument," Applied Spectroscopy, vol. 59, no. 6, pp. 769$775,2005$.

[73] E. Nyfors, "Subsurface Sensing Technologies and Applications." Springer, Germany, 2000 .

[74] K. Fuentes and K. Donaldson, "3D Scanning and Modeling," 2016 NCUR, 2016.

[75] A. Rosset, L. Spadola, and O. Ratib, "OsiriX: an open-source software for navigating in multidimensional DICOM images,” Journal of digital imaging, vol. 17, no. 3, pp. 205-216, 2004.

[76] A. Fedorov, R. Beichel, J. Kalpathy-Cramer, J. Finet, J.-C. Fillion-Robin, S. Pujol, C. Bauer, D. Jennings, F. Fennessy, M. Sonka, and others, "3D Slicer as an image computing platform for the Quantitative Imaging Network," Magnetic resonance imaging, vol. 30, no. 9, pp. 1323-1341, 2012.

[77] K. W. Eliceiri, M. R. Berthold, I. G. Goldberg, L. Ibáñez, B. S. Manjunath, M. E. Martone, R. F. Murphy, H. Peng, A. L. Plant, B. Roysam, and others, "Biological imaging software tools," Nature methods, vol. 9, no. 7, p. 697, 2012. 
[78] M. J. McAuliffe, F. M. Lalonde, D. McGarry, W. Gandler, K. Csaky, and B. L. Trus, "Medical image processing, analysis and visualization in clinical research," in ComputerBased Medical Systems, 2001. CBMS 2001. Proceedings. 14th IEEE Symposium on, 2001, pp. 381-386.

[79] H. Chan, (2017, Apr 5). Personal Interview.

[80] R. Scona, M. Jaimez, Y. R. Petillot, M. Fallon, and D. Cremers, "StaticFusion: Background Reconstruction for Dense RGB-D SLAM in Dynamic Environments," 2018.

[81] C. Day-Black, "Gamification: An Innovative Teaching-Learning Strategy for the Digital Nursing Students in a Community Health Nursing Course.," ABNF Journal, vol. 26, no. 4, 2015.

[82] J.-M. Koivisto, H. Niemi, J. Multisilta, and E. Eriksson, "Nursing students' experiential learning processes using an online 3D simulation game," Education and Information Technologies, vol. 22, no. 1, pp. 383-398, 2017.

[83] H. M. Johnsen, M. Fossum, P. Vivekananda-Schmidt, A. Fruhling, and Å. Slettebø, "Teaching clinical reasoning and decision-making skills to nursing students: design, development, and usability evaluation of a serious game," International journal of medical informatics, vol. 94, pp. 39-48, 2016.

[84] E. A. Boyle, T. Hainey, T. M. Connolly, G. Gray, J. Earp, M. Ott, T. Lim, M. Ninaus, C. Ribeiro, and J. Pereira, "An update to the systematic literature review of empirical evidence of the impacts and outcomes of computer games and serious games," Computers \& Education, vol. 94, pp. 178-192, 2016.

[85] F. Ricciardi and L. T. D. Paolis, "A comprehensive review of serious games in health professions," International Journal of Computer Games Technology, vol. 2014, p. 9, 2014.

[86] A. Poplin, "Digital serious game for urban planning:"B3-Design your Marketplace!," Environment and Planning B: Planning and Design, vol. 41, no. 3, pp. 493-511, 2014.

[87] P. Petridis, I. Dunwell, S. De Freitas, and D. Panzoli, "An engine selection methodology for high fidelity serious games," in Games and Virtual Worlds for Serious Applications (VS-GAMES), 2010 Second International Conference on, 2010, pp. 27-34.

[88] H. Hendratman, "The Magic of Blender 3D Modelling," Bandung: Informatika, 2015.

[89] P. Cignoni, M. Callieri, M. Corsini, M. Dellepiane, F. Ganovelli, and G. Ranzuglia, "Meshlab: an open-source mesh processing tool.," in Eurographics Italian Chapter Conference, 2008, vol. 2008, pp. 129-136.

[90] W. Goldstone, Unity game development essentials. Packt Publishing Ltd, 2009.

[91] K. Popovici and P. J. Mosterman, Real-time simulation technologies: principles, methodologies, and applications. CRC Press, 2017.

[92] A. D. Wilson, S. Izadi, O. Hilliges, A. Garcia-Mendoza, and D. Kirk, "Bringing physics to the surface," in Proceedings of the 21st annual ACM symposium on User interface software and technology, 2008, pp. 67-76.

[93] R. Wang, S. DeMaria Jr, A. Goldberg, and D. Katz, "A systematic review of serious games in training health care professionals," Simulation in Healthcare, vol. 11, no. 1, pp. 41-51, 2016.

[94] W. Barnat, R. Panowicz, T. Niezgoda, and P. Dybcio, "Numerical analysis of IED detonation effect on steel plate," acta mechanica et automatica, vol. 6, no. 1, pp. 10-12, 2012. 
[95] D. P. Holm, "Hydraulic ram shock wave and cavitation effects on aircraft fuel cell survivability.," Monterey, California. Naval Postgraduate School, 1973.

[96] M. Aziz, W. Kuntjoro, and N. David, "Terminal ballistic experimental analysis of an empty and full water tank," a Chapter in the WIT Transactions on Built Environment, vol. 141, pp. 191-203, 2014.

[97] D. Varas, J. López-Puente, and R. Zaera, "Experimental analysis of fluid-filled aluminium tubes subjected to high-velocity impact," International Journal of Impact Engineering, vol. 36, no. 1, pp. 81-91, 2009.

[98] F. Zhang, K. Gerrard, and R. C. Ripley, "Reaction mechanism of aluminum-particle-air detonation," Journal of propulsion and power, vol. 25, no. 4, p. 845, 2009.

[99] F. Zhang, D. Frost, P. Thibault, and S. Murray, "Explosive dispersal of solid particles," Shock Waves, vol. 10, no. 6, pp. 431-443, 2001.

[100] K. Balakrishnan, F. Genin, D. V. Nance, and S. Menon, "Numerical study of blast characteristics from detonation of homogeneous explosives," Shock Waves, vol. 20, no. 2, pp. 147-162, 2010.

[101] H. G. Weller, G. Tabor, H. Jasak, and C. Fureby, "A tensorial approach to computational continuum mechanics using object-oriented techniques," Computers in physics, vol. 12, no. 6, pp. 620-631, 1998.

[102] C. Dunfield and M. Running, (2018, Mar 3). Personal Interview.

[103] A. Olesen, (2017, May 5). Personal Interview.

[104] R. L. Simpson and C. O. Pruneda, "Method for fabricating non-detonable explosive simulants." Google Patents, 1995.

[105] D. Detection, “The DSA Advantage | Superior IED Training Aids \& ETD Training | DSA Detection.".

[106] A. A. of Physicists in Medicine (AAPM) and others, "Adult routine head CT protocols." Version, 2012.

[107] Philips, "Philips CT Big Bore." https://www.usa.philips.com/healthcare/, 2018.

[108] S. Singh and M. K. Kalra, "Standardized CT protocols and nomenclature: better, but not yet there," Pediatric radiology, vol. 44, no. 3, pp. 440-443, 2014.

[109] P. Allisy-Roberts and J. R. Williams, Farr's physics for medical imaging. Elsevier Health Sciences, 2007.

[110] S. Leng, L. Yu, J. Wang, J. G. Fletcher, C. A. Mistretta, and C. H. McCollough, "Noise reduction in spectral CT: Reducing dose and breaking the trade-off between image noise and energy bin selection," Medical physics, vol. 38, no. 9, pp. 4946-4957, 2011.

[111] D. L. Chin, (2017, Nov 30). Personal Interview.

[112] W. A. Kalender, R. Hebel, and J. Ebersberger, "Reduction of CT artifacts caused by metallic implants.," Radiology, vol. 164, no. 2, pp. 576-577, 1987.

[113] K. T. Block, M. Uecker, and J. Frahm, "Suppression of MRI truncation artifacts using total variation constrained data extrapolation," International journal of biomedical imaging, vol. 2008, 2008.

[114] S. Nawaz, J. Fu, and D. Fan, "Metal artifacts reduction in X-ray CT based on segmentation and forward-projection," Bio-medical materials and engineering, vol. 24, no. 6, pp. 32873293, 2014.

[115] T. Ju, F. Losasso, S. Schaefer, and J. Warren, "Dual contouring of hermite data," in ACM transactions on graphics (TOG), 2002, vol. 21, no. 3, pp. 339-346. 
[116] T. McInerney and D. Tran, "Aperio: a system for visualizing 3D anatomy data using virtual mechanical tools," in International Symposium on Visual Computing, 2015, pp. 797-808.

[117] K. Shimada and D. C. Gossard, "Bubble mesh: automated triangular meshing of nonmanifold geometry by sphere packing," in Proceedings of the third ACM symposium on Solid modeling and applications, 1995, pp. 409-419.

[118] D. Group, "Defense and Security Speciality Equipement." https://proparms.com/, 2017.

[119] M. Young, (2018, Feb 15). Personal Interview.

[120] C. Wong, M. Daniel, and J. Rongong, "Energy dissipation prediction of particle dampers," Journal of Sound and Vibration, vol. 319, no. 1-2, pp. 91-118, 2009.

[121] S. Luding, "Collisions \& contacts between two particles," in Physics of dry granular media, Springer, 1998, pp. 285-304.

[122] G. Parker, "Projectile motion with air resistance quadratic in the speed," American Journal of Physics, vol. 45, no. 7, pp. 606-610, 1977.

[123] W. McDermott, "Distance Tool.” Unity Asset Store, 2018.

[124] B. Bergeron, "Developing serious games (game development series)," 2006.

[125] Z. Rumboldt, W. Huda, and J. All, "Review of portable CT with assessment of a dedicated head CT scanner," American Journal of Neuroradiology, vol. 30, no. 9, pp. 1630-1636, 2009. 